\title{
Towards geometric D6-brane model building on non-factorisable toroidal $\mathbb{Z}_{4}$-orbifolds
}

\author{
Mikel Berasaluce-González, Gabriele Honecker and Alexander Seifert \\ PRISMA Cluster of Excellence $\&$ Institut für Physik (WA THEP), \\ Johannes-Gutenberg-Universität, D-55099 Mainz, Germany \\ E-mail: mberasal@uni-mainz.de, Gabriele.Honecker@uni-mainz.de, \\ alseifer@uni-mainz.de
}

ABSTRACT: We present a geometric approach to D-brane model building on the nonfactorisable torus backgrounds of $T^{6} / \mathbb{Z}_{4}$, which are $A_{3} \times A_{3}$ and $A_{3} \times A_{1} \times B_{2}$. Based on the counting of 'short' supersymmetric three-cycles per complex structure vev, the number of physically inequivalent lattice orientations with respect to the anti-holomorphic involution $\mathcal{R}$ of the Type IIA $/ \Omega \mathcal{R}$ orientifold can be reduced to three for the $A_{3} \times A_{3}$ lattice and four for the $A_{3} \times A_{1} \times B_{2}$ lattice. While four independent three-cycles on $A_{3} \times A_{3}$ cannot accommodate phenomenologically interesting global models with a chiral spectrum, the eight-dimensional space of three-cycles on $A_{3} \times A_{1} \times B_{2}$ is rich enough to provide for particle physics models, with several globally consistent two- and four-generation PatiSalam models presented here.

We further show that for fractional sLag three-cycles, the compact geometry can be rewritten in a $\left(T^{2}\right)^{3}$ factorised form, paving the way for a generalisation of known CFT methods to determine the vector-like spectrum and to derive the low-energy effective action for open string states.

Keywords: D-branes, Superstring Vacua, Differential and Algebraic Geometry, Supersymmetry and Duality

ARXIV EPRINT: 1606.04926 


\section{Contents}

1 Introduction 1

2 Non-factorisable $T^{\mathbf{6}} / \mathbb{Z}_{\mathbf{4}}$ orbifold geometries $\quad 3$

$2.1 B_{2} \times\left(A_{1}\right)^{2} \times B_{2}$

$2.2 A_{3} \times A_{3} \quad 6$

$2.3 A_{3} \times A_{1} \times B_{2} \quad 9$

3 Intersecting brane worlds $\quad \mathbf{1 5}$

3.1 Anti-holomorphic involutions $\mathcal{R}$ of $T^{6} / \mathbb{Z}_{4} \quad 16$

$\begin{array}{lll}3.1 .1 & B_{2} \times\left(A_{1}\right)^{2} \times B_{2} & 16\end{array}$

$\begin{array}{lll}3.1 .2 & A_{3} \times A_{3} & 17\end{array}$

$\begin{array}{lll}3.1 .3 & A_{3} \times A_{1} \times B_{2} & 20\end{array}$

3.2 Supersymmetric cycles 23

3.3 Pairwise relations between choices of orientifold axes 28

3.4 Cross-check: D6-branes on top of O6-planes 30

4 Factorisation of non-factorisable orbifolds 35

5 Concrete Pati-Salam models on $A_{3} \times A_{1} \times B_{2}$

6 Discussion and conclusions $\quad 40$

A More Pati-Salam models $\quad 41$

$\begin{array}{ll}\text { A.1 } 2 \text { generations } & 41\end{array}$

$\begin{array}{ll}\text { A.2 } 4 \text { generations } & 42\end{array}$

\section{Introduction}

Ever since the finding in 1985 that string theory constitutes a framework for unifying quantum field theory and gravity, which - when compactified on a Calabi-Yau-threefold or some singular limit thereof such as a toroidal orbifold - leads to $\mathcal{N}=1$ supersymmetry in four dimensions [1], the search for vacuum configurations with not only the Standard Model spectrum but also its interactions has been intensively pursued. Starting from sporadic models of the $E_{8} \times E_{8}$ heterotic string such as [2,3], by implementing systematic computer searches, large classes of vacua with particle physics spectra on $T^{6} / \mathbb{Z}_{N}$ and $T^{6} / \mathbb{Z}_{N} \times \mathbb{Z}_{M}$ orbifolds [4-6] (see also [7] for the heterotic $\mathrm{SO}(32)$ string theory) and CalabiYau manifolds [8-12] could be constructed.

With the identification of D-branes as dynamical objects in Type I and II string theory [13] in 1995, model building also opened up in these theories, which are conjectured 
to be related by S-duality $[14,15]$ and M-/F-theory duality $[16,17]$ to the vacua of the heterotic string. The virtue of D-brane model building lies in the fact that physical quantities, like e.g. the number of particle generations, are expressed in terms of topology and geometry of the compact space, which is particularly intuitive for the case of D6-branes on three-cycles in Type IIA orientifold compactifications, see e.g. [18] for a broad recent overview. This intuition, however, comes at the cost of the relatively little explored symplectic structure of generic Calabi-Yau threefolds. Since supersymmetric - or mathematically expressed special Lagrangian (sLag) - three-cycles constitute a largely unexplored area (except for [19, 20] and [21]), intersecting D6-brane models in Type IIA orientifolds have focussed on tori and toroidal orbifolds, and more specifically on backgrounds which are factorised into two-tori, $T^{6}=\left(T^{2}\right)^{3}$, see e.g. [22-24], and $\left(T^{2}\right)^{3} / \Gamma$ with Abelian point groups $\Gamma=\mathbb{Z}_{N}$ or $\mathbb{Z}_{N} \times \mathbb{Z}_{M}$. In the latter case, models with all three-cycles inherited from the underlying torus have been constructed for $\Gamma=\mathbb{Z}_{2} \times \mathbb{Z}_{2}[25-30]$ and $\mathbb{Z}_{2} \times \mathbb{Z}_{4}[31-33]$ without discrete torsion, while fractional three-cycles consisting of components inherited from the torus plus exceptional divisors at orbifold singularities have been employed for $\Gamma=\mathbb{Z}_{4}[34]$, $\mathbb{Z}_{6}[33,35-37], \mathbb{Z}_{6}^{\prime}[37-42]$ and $\mathbb{Z}_{12-I I}[43]$ with a single $\mathbb{Z}_{2}$ sector and $\Gamma=\mathbb{Z}_{2} \times \mathbb{Z}_{2}[44,45]$, $\mathbb{Z}_{2} \times \mathbb{Z}_{6}^{\prime}[45-47]$ and $\mathbb{Z}_{2} \times \mathbb{Z}_{6}[45,48-50]$ with discrete torsion with two $\mathbb{Z}_{2}$ subgroups.

All of the above mentioned types of string vacua contain a plethora of scalar fields with flat directions. While the above models with a $\mathbb{Z}_{2} \times \mathbb{Z}_{2}$ subsymmetry allow for rigid fractional three-cycles which, in the open string spectrum, provide gauge groups without brane recombination/splitting moduli in the adjoint representation, it has recently been noticed that (most) twisted complex structure moduli associated to deformations of singularities are in fact stabilised by the existence of D-branes with $\mathrm{U}(1)$ symmetries [50-53]. To further stabilise the dilaton and untwisted complex structure moduli, one usually argues that closed string background NS-NS fluxes (see [54-56] for reviews) provide a non-trivial scalar potential, see also $[57,58]$ for attempts to incorporate NS-NS fluxes on the factorisable $T^{6} / \mathbb{Z}_{4}$ orbifold and [59] for the factorisable $T^{6} / \mathbb{Z}_{6}^{\prime}$ orbifold. However, incorporating a non-trivial NS-NS flux $\mathrm{H}_{3}$ will in general violate the factorisation into two-tori and instead lead to so-called non-factorisable torus backgrounds [60-62].

Orbifolds of non-factorisable tori have, to our best knowledge, scarcely been considered in the literature. Within Type IIA orientifolds, one of the first studies of non-factorisable $\mathbb{Z}_{N}$ orbifolds can be found in [63], where special configurations of D6-branes on top of the O6-planes lead to a local cancellation of the RR tadpoles within the compact space. In [64] a similar analysis for $\mathbb{Z}_{N} \times \mathbb{Z}_{M}$ orbifolds was performed. In [65, 66] orientifolds of $T^{6} /\left(\mathbb{Z}_{2} \times \mathbb{Z}_{2}\right)$ with (non-)factorisable lattices were considered, including D6-branes which are not parallel to the O6-planes, and in [43], three-cycles on the $D_{4} \times A_{2}$ and $D_{4} \times A_{1} \times A_{1}$ lattices with $\mathbb{Z}_{12-I}$ and $\mathbb{Z}_{12-I I}$ orbifold symmetry, respectively, were studied. Finally, in [67] the Yukawa couplings for a torus generated by a $D_{6}$ lattice were computed. Here, we will for the first time perform a thorough study of all possible $s L a g$ three-cycles on the two different non-factorisable lattice backgrounds $A_{3} \times A_{3}$ and $A_{3} \times A_{1} \times B_{2}$ of $T^{6} / \mathbb{Z}_{4}$, for which we briefly provided some preliminary results in [68].

Besides from being able to classify sLag cycles on toroidal orbifolds, these geometrically simple backgrounds are equipped with the non-negligible power of allowing for an 
explicit string quantisation and thus Conformal Field Theory (CFT) techniques, which do not only reproduce the RR tadpole cancellation conditions and chiral spectrum, but are indispensable for distinguishing gauge group enhancements $\mathrm{U}(N) \hookrightarrow \mathrm{SO}(2 N)$ or $\mathrm{USp}(2 N)$ for D-branes wrapped on orientifold invariant three-cycles and for deriving the low-energy effective action (for particle physics models based on powerful RCFT techniques see e.g. also [69-72]). So far, CFT results within the geometrically intuitive approach to Type II string model building with D-branes have been obtained only for gauge couplings at oneloop and $n$-point couplings at tree-level using bulk cycles on the factorisable six-torus $\left(T^{2}\right)^{3}$ [73-78]. For fractional cycles on $\left(T^{2}\right)^{3} / \mathbb{Z}_{N}$ and $\mathbb{Z}_{N} \times \mathbb{Z}_{M}$ orbifolds [37, 79-81], the one-loop corrections to gauge couplings and the Kähler potential at leading order could be derived, while the one-loop corrections to the open string Kähler potential are only known for bulk cycles on such orbifolds [82], see also [45, 46, 48, 83] for the distinction of $\mathrm{U}(N) \hookrightarrow \mathrm{SO}(2 N)$ versus $\mathrm{USp}(2 N)$ gauge group enhancement using one-loop gauge threshold computations. The aim of the present work is not only to generalise the geometric methods of deriving the chiral spectrum from topological intersection numbers, but also to initiate the generalisation of CFT techniques to so-called non-factorisable lattice backgrounds.

The outline of this article is as follows: in section 2 we first study the geometry of $T^{6} / \mathbb{Z}_{4}$ on non-factorisable tori, then implement anti-holomorphic involutions on the lattices to study orientifolds of Type IIA string theory in section 3, after which we proceed to discuss first hints on physical equivalences among different choices, and finally we study supersymmetric D6-branes wrapped on (fractional) three-cycles. In section 4, we argue that any supersymmetric fractional three-cycle on $T^{6} / \mathbb{Z}_{4}$ can be written in a factorised form, which paves the way for implementing CFT methods in order to distinguish gauge group enhancements $\mathrm{U}(N) \hookrightarrow \mathrm{SO}(2 N)$ versus $\operatorname{USp}(2 N)$ and to derive the vector-like matter spectrum. We then proceed to provide some explicit Pati-Salam models with two and four particle generations in section 5. Section 6 contains our conclusions and outlook, and appendix A contains some further explicit examples of globally consistent D6-brane configurations with chiral matter on the $A_{3} \times A_{1} \times B_{2}$ lattice.

\section{Non-factorisable $T^{6} / \mathbb{Z}_{4}$ orbifold geometries}

In this section, we discuss the three-cycle geometry on the two non-factorisable background lattices, $A_{3} \times A_{3}$ and $A_{3} \times A_{1} \times B_{2}$, of $T^{6} / \mathbb{Z}_{4}$. The $\mathbb{Z}_{4}$-action is usually encoded in the shift vector $\vec{\zeta}=\frac{1}{4}(1,-2,1)$ if the six-torus is parameterized by three complex coordinates, and more generally the $\mathbb{Z}_{4}$-action is generated by the Coxeter element $Q$ which acts on the root lattice of the corresponding orbifold spanned by the simple roots $\left\{e_{i}\right\}_{i=1, \ldots, 6}$. Furthermore, we denote the six toroidal one-cycles along the directions $\left\{e_{i}\right\}$ by $\pi_{i}$ and toroidal two- and three-cycles by $\pi_{i j}:=\pi_{i} \wedge \pi_{j}$ and $\pi_{i j k}:=\pi_{i} \wedge \pi_{j} \wedge \pi_{k}$, respectively. The Hodge numbers of all three possible lattice backgrounds - one factorisable and two non-factorisable ones are summarized in table 1 (cf. e.g. [84]). We are in particular interested in characterization of the three-homology $H_{3}\left(T^{6} / \mathbb{Z}_{4}, \mathbb{Z}\right)$ of each background lattice. This homology class contains in general the $\mathbb{Z}_{4}$-invariant bulk $\pi^{\text {bulk }}$ and exceptional three-cycles $\pi^{\text {exc }}$ as well as 


\begin{tabular}{|c|c|c|c||c|c|c|}
\hline \multicolumn{7}{|c|}{ Hodge numbers of $T^{6} / \mathbb{Z}_{4}$ orbifolds } \\
\hline Lattice & $h_{11}^{\text {bulk }}$ & $h_{11}^{\mathbb{Z}_{2}}$ & $h_{11}^{\mathbb{Z}_{4}}$ & $h_{21}^{\text {bulk }}$ & $h_{21}^{\mathbb{Z}_{2}}$ & $h_{21}^{\mathbb{Z}_{4}}$ \\
\hline$B_{2} \times\left(A_{1}\right)^{2} \times B_{2}$ & 5 & 10 & 16 & 1 & 6 & 0 \\
\hline$A_{3} \times A_{3}$ & 5 & 4 & 16 & 1 & 0 & 0 \\
\hline$A_{3} \times A_{1} \times B_{2}$ & 5 & 6 & 16 & 1 & 2 & 0 \\
\hline
\end{tabular}

Table 1. Summary of the Hodge numbers per untwisted and twisted sector of the $T^{6} / \mathbb{Z}_{4}$ orbifolds on factorisable and non-factorisable tori.

fractional linear combinations thereof, the so-called fractional three-cycles $\pi^{\text {frac }}$. The bulk three-cycles are inherited from the underlying torus and can be computed by taking the $\mathbb{Z}_{4}$-orbits thereof:

$$
\pi^{\text {bulk }}:=\sum_{i=0}^{i=3} Q^{i} \pi^{\text {torus }}
$$

The exceptional three-cycles arise for the $T^{6} / \mathbb{Z}_{4}$ orbifolds only in the $\mathbb{Z}_{2}$-twisted sector. They stem from the resolution of the $\mathbb{Z}_{2}$-invariant two-tori and can be written as a product of an exceptional two-cycle $\mathbf{e}_{\alpha \beta}$ (with $\alpha \beta$ labelling the location of the cycle) and a $\mathbb{Z}_{2^{-}}$ invariant toroidal one-cycle (plus some $\mathbb{Z}_{4}$-image). Finally, fractional three-cycles are either one-half of a bulk cycle or linear combinations of one-half of some bulk and exceptional three-cycles with the combinatorics depending on the corresponding singularities traversed by the bulk cycles as well as sign factors associated to the choice of some discrete Wilson line, or geometrically speaking the orientation the corresponding exceptional three-cycle is wrapped.

Based on the discussion in this section, we will proceed to discuss (supersymmetric) Type IIA orientifolds with O6-planes and D6-branes and the associated anti-holomorphic involution on the background geometry in section 3 .

\section{$2.1 \quad B_{2} \times\left(A_{1}\right)^{2} \times B_{2}$}

Before investigating the three-cycle geometry of the non-factorisable lattices, we briefly review three-cycles on the factorisable background on the group lattice $B_{2} \times\left(A_{1}\right)^{2} \times B_{2}$ as first discussed in [34], see e.g. also the appendix of [45] for the Hodge numbers per twist sector displayed in table 1 . Although the $B_{2}$-torus is a square-torus, we take the positive simple roots of the $B_{2}$-Lie algebra as basis of the torus lattice (see figure 1 ). The $\mathbb{Z}_{4}$-action is then generated by the Coxeter element $Q$ :

$$
Q:=\left(\begin{array}{ll}
1 & -1 \\
2 & -1
\end{array}\right) \oplus\left(\begin{array}{cc}
-1 & 0 \\
0 & -1
\end{array}\right) \oplus\left(\begin{array}{ll}
1 & -1 \\
2 & -1
\end{array}\right)
$$




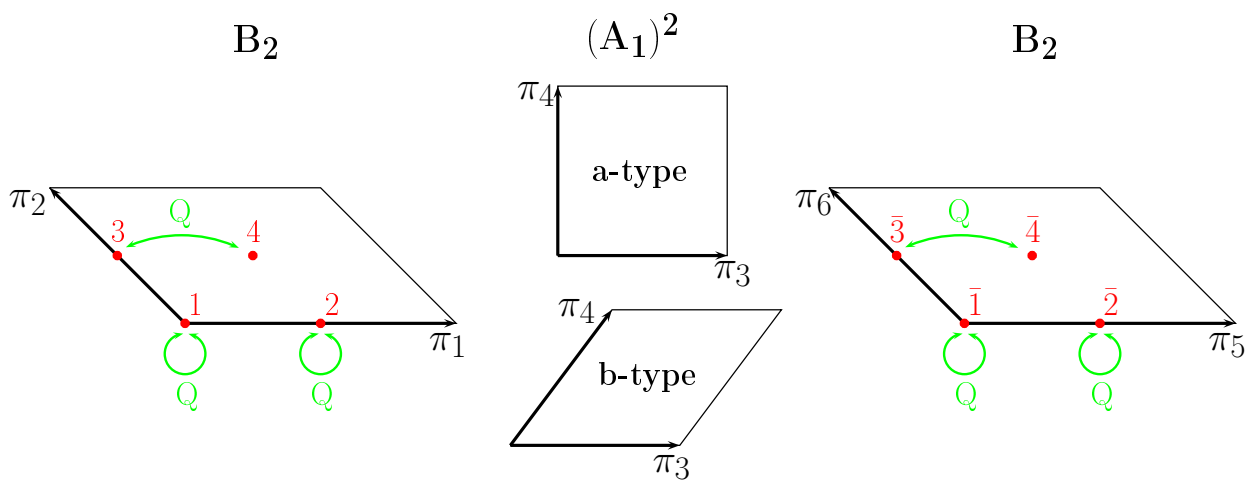

Figure 1. $T^{6} / \mathbb{Z}_{4}$-orbifold on the $B_{2} \times\left(A_{1}\right)^{2} \times B_{2}$-lattice and the $\mathbb{Z}_{2}$-invariant points (in red) on $T_{(1)}^{2} \times T_{(3)}^{2}$ with both possible shapes $\mathbf{a}$ and $\mathbf{b}$ displayed for the $\left(A_{1}\right)^{2}$ torus.

acting on three two-tori $T_{(i)}^{2}$. A basis of bulk three-cycles on the factorisable lattice is given by

$$
\begin{aligned}
& \gamma_{1}:=\sum_{k=0}^{3} Q^{k}\left(\pi_{235}+\pi_{236}\right)=2\left(\pi_{136}+\pi_{235}+2 \pi_{236}\right), \\
& \gamma_{2}:=-\sum_{k=0}^{3} Q^{k} \pi_{236}=2\left(\pi_{135}+\pi_{136}+\pi_{235}\right), \\
& \bar{\gamma}_{1}:=\sum_{k=0}^{3} Q^{k}\left(\pi_{245}+\pi_{246}\right)=2\left(\pi_{146}+\pi_{245}+2 \pi_{246}\right), \\
& \bar{\gamma}_{2}:=-\sum_{k=0}^{3} Q^{k} \pi_{246}=2\left(\pi_{145}+\pi_{146}+\pi_{245}\right) .
\end{aligned}
$$

An arbitrary bulk three-cycle can be represented by the (pairwise co-prime) toroidal wrapping numbers $\left(n^{i}, m^{i}\right)_{i=1,2,3}$,

$$
\begin{aligned}
\pi^{\mathrm{bulk}} & =\sum_{k=0}^{3} Q^{k}\left(\bigwedge_{i=1}^{3}\left(n^{i} \pi_{2 i-1}+m^{i} \pi_{2 i}\right)\right) \\
& =A n^{2} \gamma_{1}+A m^{2} \bar{\gamma}_{1}+B n^{2} \gamma_{2}+B m^{2} \bar{\gamma}_{2}
\end{aligned}
$$

where on the second line the $\mathbb{Z}_{4}$ invariant bulk wrapping numbers with

$$
A:=n^{1} m^{3}+m^{1} n^{3}-2 n^{1} n^{3}, \quad B:=n^{1} m^{3}+m^{1} n^{3}-m^{1} m^{3},
$$

have been used. The basic non-vanishing bulk intersection numbers are computed from

$$
\pi_{a}^{\text {bulk }} \circ \pi_{b}^{\text {bulk }} \equiv \frac{1}{4}\left(\sum_{i=0}^{3} Q^{i} \pi_{a}^{\text {torus }}\right) \circ\left(\sum_{i=0}^{3} Q^{i} \pi_{b}^{\text {torus }}\right)=\pi_{a}^{\text {torus }} \circ\left(\sum_{i=0}^{3} Q^{i} \pi_{b}^{\text {torus }}\right)
$$

and read

$$
\gamma_{i} \circ \bar{\gamma}_{j}=2 \delta_{i j}
$$


$A_{3}$

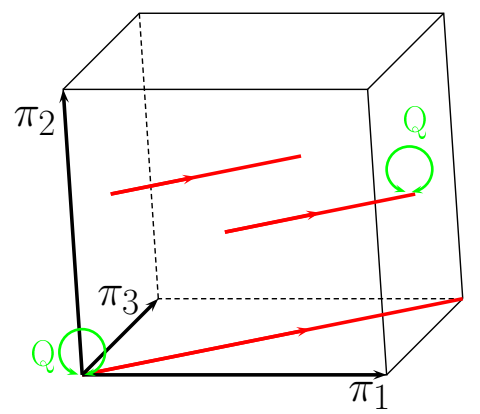

$A_{3}$

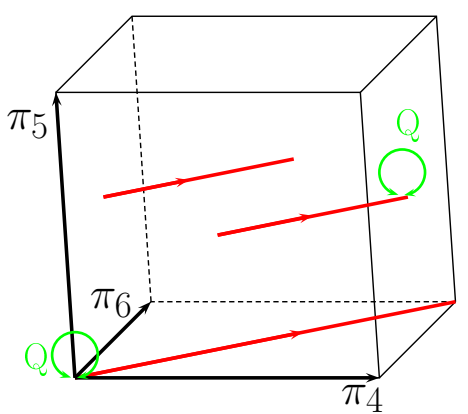

Figure 2. $T^{6} / \mathbb{Z}_{4}$-orbifold on the $A_{3} \times A_{3}$-lattice and its $\mathbb{Z}_{2}$-fixed lines (in red).

The bulk three-cycles thus do not form an unimodular basis. In addition to the bulk three-cycles, there exist twelve exceptional cycles with basis

$$
\varepsilon_{i}=\left(e_{\alpha \beta}-e_{Q(\alpha) Q(\beta)}\right) \wedge \pi_{3}, \quad \bar{\varepsilon}_{i}=\left(e_{\alpha \beta}-e_{Q(\alpha) Q(\beta)}\right) \wedge \pi_{4}
$$

(where $\alpha$ and $\beta$ denote the $\mathbb{Z}_{2}$-invariant points on the four-torus $T_{(1)}^{2} \times T_{(3)}^{2}$ and $Q(\alpha)$ and $Q(\beta)$ their $\mathbb{Z}_{4}$ images), and with non-vanishing intersection numbers

$$
\varepsilon_{i} \circ \bar{\varepsilon}_{j}=-2 \delta_{i j} .
$$

Fractional three-cycles of the form

$$
\pi^{\text {frac }}=\frac{1}{2}\left(\pi^{\text {bulk }}+(-1)^{\tau^{\mathbb{Z}_{2}}} \sum_{\text {fixed set of } i}\left[ \pm\left(n^{2} \varepsilon_{i}+m^{2} \bar{\varepsilon}_{i}\right)\right]\right),
$$

with the $\mathbb{Z}_{2}$ eigenvalue $(-1)^{\tau^{Z_{2}}}$ parametrised by $\tau^{\mathbb{Z}_{2}} \in\{0,1\}$ and the sum over four $i$ such that the product of the signs \pm gives +1 , then generate the unimodular sixteen dimensional basis of three-cycles.

\section{$2.2 \quad A_{3} \times A_{3}$}

We start the discussion of non-factorisable $\mathbb{Z}_{4}$-orbifolds with the lattice of the type $A_{3} \times A_{3}$ (see figure 2). The $\mathbb{Z}_{4}$ group acts by the Coxeter element $Q$ on the vectors $\left\{e_{i}\right\}_{i=1, \ldots, 6}$, which span the six-torus:

$$
\begin{aligned}
& Q e_{1}=e_{2}, \quad Q e_{2}=e_{3}, \quad Q e_{3}=-e_{1}-e_{2}-e_{3}, \\
& Q e_{4}=e_{5}, \quad Q e_{5}=e_{6}, \quad Q e_{6}=-e_{4}-e_{5}-e_{6},
\end{aligned}
$$

which can be written in the matrix form

$$
Q:=\left(\begin{array}{cccccc}
0 & 0 & -1 & 0 & 0 & 0 \\
1 & 0 & -1 & 0 & 0 & 0 \\
0 & 1 & -1 & 0 & 0 & 0 \\
0 & 0 & 0 & 0 & 0 & -1 \\
0 & 0 & 0 & 1 & 0 & -1 \\
0 & 0 & 0 & 0 & 1 & -1
\end{array}\right) .
$$


This action forms a discrete subgroup of $\mathrm{SU}(3)$ and thus preserves $\mathcal{N}=2$ supersymmetry in four dimensions when compactifying Type II string theory. The Hodge numbers of this orbifold are (see e.g. [84]) $h_{21}=h_{21}^{\text {untw }}+h_{21}^{\mathbb{Z}_{2}}=1+0$ and $h_{11}=h_{11}^{\text {untw }}+h_{11}^{\mathbb{Z}_{4}}+h_{11}^{\mathbb{Z}_{2}}=5+16+4$ as displayed in the middle row in table 1 . Due to $h_{21}^{\mathbb{Z}_{2}}=0$, the three-homology of this orbifold contains only (fractions of) bulk three-cycles and is four-dimensional.

For the $Q$-action to be an isometry of the lattice, one has to require the invariance of the scalar product $Q^{t} g Q=g$ [85], which restricts the shape of the metric of the six-torus to

$$
g=\left(\begin{array}{cccccc}
R_{1}^{2} & a R_{1}^{2} & -(2 a+1) R_{1}^{2} & e R_{1} R_{2} & s R_{1} R_{2} & c R_{1} R_{2} \\
a R_{1}^{2} & R_{1}^{2} & a R_{1}^{2} & d R_{1} R_{2} & e R_{1} R_{2} & s R_{1} R_{2} \\
-(2 a+1) R_{1}^{2} & a R_{1}^{2} & R_{1}^{2} & c R_{1} R_{2} & d R_{1} R_{2} & e R_{1} R_{2} \\
e R_{1} R_{2} & d R_{1} R_{2} & c R_{1} R_{2} & R_{2}^{2} & b R_{2}^{2} & -(2 b+1) R_{2}^{2} \\
s R_{1} R_{2} & e R_{1} R_{2} & d R_{1} R_{2} & b R_{2}^{2} & R_{2}^{2} & b R_{2}^{2} \\
c R_{1} R_{2} & s R_{1} R_{2} & e R_{1} R_{2} & -(2 b+1) R_{2}^{2} & b R_{2}^{2} & R_{2}^{2}
\end{array}\right)
$$

with $s:=-(c+d+e)$. The moduli $R_{1}$ and $R_{2}$ describe the radii of $A_{3} \times A_{3}$ whereas $a(b)$ specifies the cosine of the angle between the vectors $e_{1}$ and $e_{2}\left(e_{4}\right.$ and $\left.e_{5}\right)$ and $c, d$ and $e$ specify the cosines of the angles between $e_{4}$ and the vectors $e_{3}, e_{2}$ and $e_{1}$, respectively.

To describe the three-cycles on the six-torus $T^{6}$ we use the usual notation $\pi_{i k l}=$ $\pi_{i} \wedge \pi_{k} \wedge \pi_{l}$. Due to the action of $Q$, it suffices to consider the three-cycles which wrap a two-cycle on one $A_{3}$-torus and a one-cycle on the other one. Such cycles can a priori be described by twelve wrapping numbers $\left(m^{i}, n^{i}, p^{i}, q^{i}, r^{i}, s^{i}\right)^{i=1,2}$ :

$\pi^{\text {torus }}:=\left(m^{1} \pi_{1}+n^{1} \pi_{2}+p^{1} \pi_{3}\right) \wedge\left(m^{2} \pi_{1}+n^{2} \pi_{2}+p^{2} \pi_{3}+q^{2} \pi_{4}+r^{2} \pi_{5}+s^{2} \pi_{6}\right) \wedge\left(q^{1} \pi_{4}+r^{1} \pi_{5}+s^{1} \pi_{6}\right)$.

By taking orbits of the $Q$-action, the basis of $\mathbb{Z}_{4}$-invariant three-cycles is given by

$$
\begin{aligned}
\gamma_{1} & :=\sum_{i=0}^{3} Q^{i} \pi_{124}=-\pi_{125}-\pi_{126}-\pi_{134}-\pi_{135}+\pi_{235}+\pi_{236}, \\
\gamma_{2} & :=\sum_{i=0}^{3} Q^{i} \pi_{125}=\pi_{124}+\pi_{125}-\pi_{135}-\pi_{136}-\pi_{234}-\pi_{235}, \\
\bar{\gamma}_{1} & :=\sum_{i=0}^{3} Q^{i} \pi_{145}=-\pi_{146}-\pi_{245}-\pi_{246}+\pi_{256}-\pi_{345}+\pi_{356}, \\
\bar{\gamma}_{2} & :=\sum_{i=0}^{3} Q^{i} \pi_{245}=\pi_{145}-\pi_{156}+\pi_{245}-\pi_{246}-\pi_{256}-\pi_{346} .
\end{aligned}
$$

Using the ansatz (2.14) for a toroidal three-cycle, we can compute the corresponding bulk cycle with the orbifold map $\sum_{i=0}^{3} Q^{i}$ and decompose it in the basis (2.15):

$$
\begin{aligned}
\pi^{\mathrm{bulk}}= & \left(A_{1}\left(q^{1}-s^{1}\right)-A_{2}\left(q^{1}+r^{1}-s^{1}\right)+A_{3} r^{1}\right) \gamma_{1} \\
& +\left(A_{1} r^{1}+A_{2}\left(q^{1}-r^{1}-s^{1}\right)-A_{3}\left(q^{1}-s^{1}\right)\right) \gamma_{2} \\
& +\left(B_{1}\left(m^{1}-p^{1}\right)-B_{2}\left(m^{1}+n^{1}-p^{1}\right)+B_{3} n^{1}\right) \bar{\gamma}_{1} \\
& +\left(B_{1} n^{1}+B_{2}\left(m^{1}-n^{1}-p^{1}\right)-B_{3}\left(m^{1}-p^{1}\right)\right) \bar{\gamma}_{2}
\end{aligned}
$$


with

$$
\begin{array}{lll}
A_{1}:=m^{1} n^{2}-n^{1} m^{2}, & A_{2}:=m^{1} p^{2}-p^{1} m^{2}, & A_{3}:=n^{1} p^{2}-p^{1} n^{2}, \\
B_{1}:=q^{2} r^{1}-r^{2} q^{1}, & B_{2}:=q^{2} s^{1}-s^{2} q^{1}, & B_{3}:=r^{2} s^{1}-s^{2} r^{1} .
\end{array}
$$

It is easy to verify that the non-vanishing intersection numbers between the basis elements (2.15) are given by

$$
\gamma_{i} \circ \bar{\gamma}_{j}=-\delta_{i j}
$$

where we used analogously to the factorised lattice background in section 2.1 that we can define the intersection number between two toroidal three-cycles $\pi_{a}^{\text {torus }}$ and $\pi_{b}^{\text {torus }}$ on $T^{6}$ as $\pi_{a}^{\text {torus }} \circ \pi_{b}^{\text {torus }}=\pi_{a}^{\text {torus }} \wedge \pi_{b}^{\text {torus }} / \operatorname{Vol}\left(T^{6}\right)$ to arrive at the intersection number between two $\mathbb{Z}_{4}$-invariant bulk three-cycles

$$
\pi_{a} \circ \pi_{b}=\frac{1}{4}\left(\sum_{i=0}^{3} Q^{i} \pi_{a}^{\text {torus }}\right) \circ\left(\sum_{i=0}^{3} Q^{i} \pi_{b}^{\text {torus }}\right) .
$$

Therefore, $\left\{\gamma_{1}, \gamma_{2}, \bar{\gamma}_{1}, \bar{\gamma}_{2}\right\}$ already builds the unimodular basis.

Despite this and the fact that this orbifold does not have any exceptional three-cycles, in order to compare with the other non-factorisable lattice in section 2.3 below, we can consider the special class of three-cycles, for which the toroidal building blocks are $\mathbb{Z}_{2-}$ invariant, i.e. fractional three-cycles ${ }^{1}$

$$
\pi^{\mathrm{frac}}=\frac{1}{2} \pi^{\mathrm{bulk}}
$$

Using the $Q$-transformation of the wrapping numbers

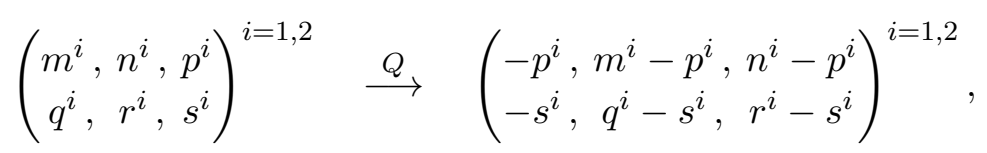

we obtain the following condition. Any toroidal three-cycle $\pi^{\text {torus }}$ of the form (2.14) is $\mathbb{Z}_{2}$-invariant if and only if

$$
\begin{aligned}
r^{1}\left(A_{1}+A_{3}\right) & =0, & n^{1}\left(B_{1}+B_{3}\right) & =0, \\
\left(s^{1}-r^{1}\right) A_{3} & =q^{1} A_{1}, & \left(p^{1}-n^{1}\right) B_{3} & =m^{1} B_{1}, \\
\left(A_{1}+A_{3}\right)\left(s^{1}-q^{1}\right) & =0, & \left(B_{1}+B_{3}\right)\left(m^{1}-p^{1}\right) & =0, \\
\left(A_{1}+A_{3}\right) s^{1} & =\left(q^{1}-r^{1}+s^{1}\right) A_{2}, & \left(B_{1}+B_{3}\right) p^{1} & =\left(m^{1}-n^{1}+p^{1}\right) B_{2} .
\end{aligned}
$$

Among this special class of three-cycles from $\mathbb{Z}_{2}$-invariant toroidal cycles, there are also those that satisfy $Q \pi^{\text {torus }}=-\pi^{\text {torus }}$. They do not contribute to the bulk cycles, or in

\footnotetext{
${ }^{1}$ This construction will be used in the next sections. We will see that if both $A_{3}$-tori are orthogonal to each other, any fractional three-cycle is Lagrangian.

Note also that for $T^{6} /\left(\mathbb{Z}_{2} \times \mathbb{Z}_{2}\right)$ without discrete torsion, the unimodular basis is constructed analogously using $\mathbb{Z}_{2} \times \mathbb{Z}_{2}$-invariant three-cycles $\pi^{\text {torus }}=\frac{1}{4} \pi^{\text {bulk }}$, cf. e.g. [86]. However, since $Q$ as defined in (2.12) permutes toroidal one-cycles on $T^{6} / \mathbb{Z}_{4}$ non-trivially, here we have to restrict to a special subclass of all $a$ priori allowed bulk three-cycles.
} 
other words are trivial in the $H_{3}\left(T^{6} / \mathbb{Z}_{4}, \mathbb{Z}\right)$ homology. Taking into account this fact, we can reduce the conditions $(2.21)$ to

$$
\begin{aligned}
A_{1}+A_{3} & =0, & B_{1}+B_{3} & =0, \\
\left(q^{1}-r^{1}+s^{1}\right) A_{i} & =0, & \left(m^{1}-n^{1}+p^{1}\right) B_{i} & =0 \text { for all } i .
\end{aligned}
$$

Note that due to the above $\mathbb{Z}_{2}$-invariance constraints on the wrapping numbers, the basis of these fractional three-cycles coincides with the basis $\left\{\gamma_{1}, \gamma_{2}, \bar{\gamma}_{1}, \bar{\gamma}_{2}\right\}$.

Let us for example consider the toroidal three-cycle $\pi=\left(\pi_{1}+\pi_{2}\right) \wedge\left(\pi_{1}+\pi_{3}\right) \wedge\left(\pi_{4}-\pi_{6}\right)$. Obviously, it is $\mathbb{Z}_{2}$-invariant and gives rise to the fractional three-cycle $\pi^{\text {frac }}=\pi+Q \pi=$ $-2 \gamma_{1}$ on $T^{6} / \mathbb{Z}_{4}$. Although the original cycle has coprime wrapping numbers and no other toroidal three-cycle $\pi^{\prime}$ exists such that $\pi=2 \pi^{\prime}$, the corresponding bulk cycle $-2 \gamma_{1}$ is non-coprime. This will play a role for computing the gauge group in the section 3.4.

\section{$2.3 \quad A_{3} \times A_{1} \times B_{2}$}

Now we consider three-cycles on $T^{6} / \mathbb{Z}_{4}$ with the lattice of type $A_{3} \times A_{1} \times B_{2}$. The $\mathbb{Z}_{4}$-action is generated by the Coxeter element which acts on the root lattice spanned by the simple roots $\left\{e_{i}\right\}_{i=1, \ldots, 6}$ in the following way

$$
\begin{array}{lll}
Q e_{1}=e_{2}, & Q e_{2}=e_{3}, & Q e_{3}=-e_{1}-e_{2}-e_{3}, \\
Q e_{4}=-e_{4}, & Q e_{5}=e_{5}+2 e_{6}, & Q e_{6}=-e_{5}-e_{6},
\end{array}
$$

which can be cast in the matrix form

$$
Q:=\left(\begin{array}{cccccc}
0 & 0 & -1 & 0 & 0 & 0 \\
1 & 0 & -1 & 0 & 0 & 0 \\
0 & 1 & -1 & 0 & 0 & 0 \\
0 & 0 & 0 & -1 & 0 & 0 \\
0 & 0 & 0 & 0 & 1 & -1 \\
0 & 0 & 0 & 0 & 2 & -1
\end{array}\right)
$$

Again, this action corresponds to a discrete subgroup of SU(3), and thus preserves $\mathcal{N}=2$ supersymmetry in four dimensions when considering Type II string theory compactifications. The Hodge numbers of this orbifold are [84] $h_{21}=h_{21}^{\text {untw }}+h_{21}^{\mathbb{Z}_{2}}=1+2$ and $h_{11}=h_{11}^{\text {untw }}+h_{11}^{\mathbb{Z}_{4}}+h_{11}^{\mathbb{Z}_{2}}=5+16+6$ (see table 1 ). Thus, we expect four bulk and four exceptional three-cycles on this orbifold.

From solving the equation $Q^{t} g Q=g$ we obtain

$$
g:=e_{i} \cdot e_{j}=\left(\begin{array}{cccccc}
R_{3}^{2} & a R_{3}^{2} & -(1+2 a) R_{3}^{2} & d R_{3} R_{1} & b R_{3} R_{2} & c R_{3} R_{2} \\
a R_{3}^{2} & R_{3}^{2} & a R_{3}^{2} & -d R_{3} R_{1} & -(b+2 c) R_{3} R_{2} & (b+c) R_{3} R_{2} \\
-(1+2 a) R_{3}^{2} & a R_{3}^{2} & R_{3}^{2} & d R_{3} R_{1} & -b R_{3} R_{2} & -c R_{3} R_{2} \\
d R_{3} R_{1} & -d R_{3} R_{1} & d R_{3} R_{1} & R_{1}^{2} & 0 & 0 \\
b R_{3} R_{2} & -(b+2 c) R_{3} R_{2} & -b R_{3} R_{2} & 0 & 2 R_{2}^{2} & -R_{2}^{2} \\
c R_{3} R_{2} & (b+c) R_{3} R_{2} & -c R_{3} R_{2} & 0 & -R_{2}^{2} & R_{2}^{2}
\end{array}\right) .
$$

The real positive moduli $R_{3}, R_{1}$ and $R_{2}$ describe the radii of $A_{3} \times A_{1} \times B_{2}$, respectively, and $a, b, c$ and $d$ specify the cosines of angles between the vectors of the lattice. More precisely, $a$ is the cosine of the angle between the vectors $e_{1}$ and $e_{2}, d$ the cosine of the angle between $e_{1}$ and $e_{4}, b(c)$ is the cosine of the angle between $e_{1}$ and $e_{5}\left(e_{6}\right)$. 
Bulk three-cycles. We make the ansatz that any toroidal three-cycle is factorisable in the sense that it can be characterised a priori by ten wrapping numbers $\left(m^{1}, n^{1}, p^{1}, q^{1}\right) \times$ $\left(m^{2}, n^{2}, p^{2}, q^{2}\right) \times\left(m^{3}, n^{3}\right)$ and written as

$$
\pi^{\text {torus }}:=\bigwedge_{i=1}^{2}\left(m^{i} \pi_{1}+n^{i} \pi_{2}+p^{i} \pi_{3}+q^{i} \pi_{4}\right) \wedge\left(m^{3} \pi_{5}+n^{3} \pi_{6}\right) .
$$

The last doublet $\left(\mathrm{m}^{3}, \mathrm{n}^{3}\right)$ gives us the one-cycle on the $B_{2}$-torus. The two quadruplets $\left(m^{i}, n^{i}, p^{i}, q^{i}\right)$ parametrise the two-cycle on $A_{3} \times A_{1}$. It is easy to see that the representation of three-cycles by this ansatz is not unique, i.e. the same three-cycle can be described by different wrapping numbers, e.g. when permuting the indices $i=1$ and 2 .

By taking orbits of the $Q$-action, we can define a basis of the $\mathbb{Z}_{4}$-invariant bulk threecycles

$$
\begin{aligned}
& \gamma_{1}:=-\sum_{i=0}^{3} Q^{i} \pi_{136}=2\left(\pi_{125}+\pi_{126}-\pi_{136}-\pi_{235}-\pi_{236}\right), \\
& \gamma_{2}:=-\sum_{i=0}^{3} Q^{i} \pi_{125}=2\left(\pi_{126}+\pi_{135}+\pi_{136}-\pi_{236}\right), \\
& \bar{\gamma}_{1}:=\sum_{i=0}^{3} Q^{i} \pi_{146}=\pi_{145}+2 \pi_{146}+2 \pi_{245}+2 \pi_{246}+\pi_{345}, \\
& \bar{\gamma}_{2}:=\sum_{i=0}^{3} Q^{i} \pi_{246}=-\pi_{145}+2 \pi_{246}+\pi_{345}+2 \pi_{346} .
\end{aligned}
$$

Note that here the linear combinations $\frac{1}{2}\left(\gamma_{1} \pm \gamma_{2}\right)$ are also bulk cycles. The decomposition in the basis $\left\{\gamma_{1,2}, \bar{\gamma}_{1,2}\right\}$ of any bulk three-cycle inherited from a toroidal one of the type (2.26) is given by

$$
\begin{aligned}
\pi^{\text {bulk }}= & P \gamma_{1}+Q \gamma_{2}+\bar{P} \bar{\gamma}_{1}+\bar{Q} \bar{\gamma}_{2} \\
= & {\left[\left(A_{2}-A_{3}\right) m^{3}+\frac{1}{2}\left(A_{1}-2 A_{2}+A_{3}\right) n^{3}\right] \gamma_{1}+\left[\left(A_{2}-A_{1}\right) m^{3}+\frac{1}{2}\left(A_{1}-A_{3}\right) n^{3}\right] \gamma_{2} } \\
& +\left[\left(-B_{1}+B_{2}+B_{3}\right) m^{3}+\left(B_{1}-B_{3}\right) n^{3}\right] \bar{\gamma}_{1}+\left[\left(-B_{1}-B_{2}+B_{3}\right) m^{3}+B_{2} n^{3}\right] \bar{\gamma}_{2},
\end{aligned}
$$

with

$$
\begin{array}{ll}
A_{1}:=m^{1} n^{2}-n^{1} m^{2}, & B_{1}:=m^{1} q^{2}-q^{1} m^{2}, \\
A_{2}:=m^{1} p^{2}-p^{1} m^{2}, & B_{2}:=n^{1} q^{2}-q^{1} n^{2}, \\
A_{3}:=n^{1} p^{2}-p^{1} n^{2}, & B_{3}:=p^{1} q^{2}-q^{1} p^{2} .
\end{array}
$$

Using the formula for bulk intersection numbers (2.18), we obtain for the bulk basis of (2.27)

$$
\gamma_{i} \circ \bar{\gamma}_{j}=-2 \delta_{i j}, \quad \gamma_{i} \circ \gamma_{j}=\bar{\gamma}_{i} \circ \bar{\gamma}_{j}=0,
$$

which shows that the integral basis of bulk three-cycles is not unimodular. 


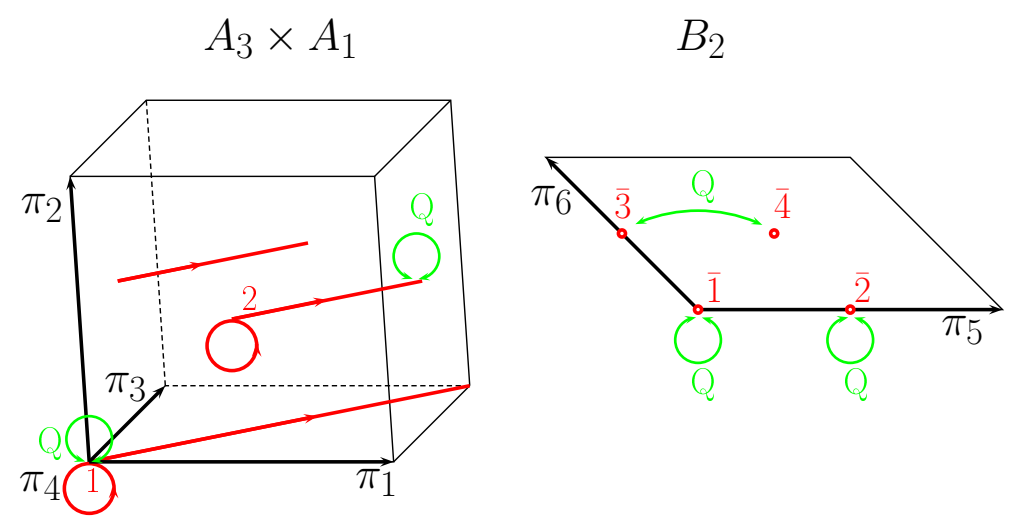

Figure 3. $T^{6} / \mathbb{Z}_{4}$-orbifold on the $A_{3} \times A_{1} \times B_{2}$-lattice and its $\mathbb{Z}_{2}$-fixed lines (in red).

Exceptional three-cycles. Besides the four bulk three-cycles $\gamma_{i}, \bar{\gamma}_{i}$ (with $i=1,2$ ), there are also four exceptional three-cycles appearing in the $\mathbb{Z}_{2}$ twisted sector of the orbifold. It is easy to see that $Q^{2}$ acts trivially on the sub-manifold $\left(\pi_{1}+\pi_{3}\right) \wedge \pi_{4}$. One can show that there are eight such $Q^{2}$-invariant sub-manifolds, which are indicated in red in figure 3. We numerate them by $i \bar{j}$ where the first index denotes the $\mathbb{Z}_{2}$-invariant two-tori $(1,2)$ on the $A_{3} \times A_{1}$-torus and the second one the $\mathbb{Z}_{2}$-invariant points $(\overline{1}, \overline{2}, \overline{3}, \overline{4})$ on the $B_{2}$-torus. These $Q^{2}$-invariant sub-manifolds can be arranged in six congruence classes (under the $Q$-action): $\{1 \overline{1}\},\{1 \overline{2}\},\{1 \overline{3}, 1 \overline{4}\},\{2 \overline{1}\},\{2 \overline{2}\},\{2 \overline{3}, 2 \overline{4}\}$.

The resolution of these six $\mathbb{Z}_{2}$-singular sub-manifolds gives rise to six four-dimensional sub-manifolds with the topology $S^{2} \times T^{2}$ in accordance with $h_{22}^{\mathbb{Z}_{2}} \stackrel{\text { Poincaré }}{=} h_{11}^{\mathbb{Z}_{2}}=6$ in table 1 , where the exceptional two-cycle $\mathbf{e}_{i \bar{j}}$ describes the $S^{2}$-part, and the two one-cycles $\pi_{1}+\pi_{3}$ and $\pi_{4}$ span a two-torus. The index of $\mathbf{e}_{i \bar{j}}$ is inherited from the numeration of the $\mathbb{Z}_{2^{-}}$ invariant two-tori. Finally by splitting $T^{2}$ into one-cycles $\pi_{1}+\pi_{3}$ and $\pi_{4}$, we construct $Q$-invariant exceptional three-cycles. Due to the anti-symmetric action of $Q$ on the onecycles, only the exceptional two-cycles $\mathbf{e}_{1 \overline{3}}, \mathbf{e}_{1 \overline{4}}, \mathbf{e}_{2 \overline{3}}, \mathbf{e}_{2 \overline{4}}$ provide non-trivial results in the construction. Thus, the exceptional three-cycles are:

$$
\begin{array}{ll}
\gamma_{3}:=\left(\mathbf{e}_{1 \overline{3}}-\mathbf{e}_{1 \overline{4}}\right) \wedge\left(\pi_{1}+\pi_{3}\right), & \bar{\gamma}_{3}:=\left(\mathbf{e}_{1 \overline{3}}-\mathbf{e}_{1 \overline{4}}\right) \wedge \pi_{4}, \\
\gamma_{4}:=\left(\mathbf{e}_{2 \overline{3}}-\mathbf{e}_{2 \overline{4}}\right) \wedge\left(\pi_{1}+\pi_{3}\right), & \bar{\gamma}_{4}:=\left(\mathbf{e}_{2 \overline{3}}-\mathbf{e}_{2 \overline{4}}\right) \wedge \pi_{4},
\end{array}
$$

with the intersection numbers

$$
\gamma_{i} \circ \bar{\gamma}_{j}=2 \delta_{i j}, \quad \gamma_{i} \circ \gamma_{j}=\bar{\gamma}_{i} \circ \bar{\gamma}_{j}=0 \quad i=3,4 .
$$

Since the intersection form of the $\gamma_{i}{ }^{\prime}$ s, and $\bar{\gamma}_{i}$ 's $(i=1,2,3,4)$ is not unimodular, these three-cycles do not form the minimal integral basis. We thus have to consider fractional three-cycles, which can consist of half a bulk cycle and simultaneously of half an exceptional cycle.

Fractional three-cycles and their integral basis. In order to write down an integral basis for the three-cycles such that the intersection form is unimodular, we start by specifying the construction of fractional cycles, which are $\mathbb{Z}_{2}$-invariant, analogously to the 
factorisable case reviewed in section 2.1. The fractional cycles can wrap half a bulk cycle and half an exceptional one

$$
\pi^{\mathrm{frac}}=\frac{1}{2} \pi^{\mathrm{bulk}}+\frac{1}{2} \pi^{\mathrm{exc}}
$$

Motivated by the factorisable orbifold, we apply the well-known techniques of construction of the fractional cycles to our case. The non-factorisable structure of the lattice gives rise to some differences and therefore some modification of these techniques is needed.

In the case of the factorisable torus $T_{1}^{2} \times T_{2}^{2} \times T_{3}^{2}$, every $\mathbb{Z}_{2}$-invariant three-cycle passes through two $\mathbb{Z}_{2}$-invariant points per $T^{2}$ (here $T_{1}^{2} \times T_{3}^{2}$ ) and contains a $\mathbb{Z}_{2}$-invariant onecycle on the remaining two-torus (here $T_{2}^{2}$ ). In the $A_{3} \times A_{3}$-case we saw that this does not hold true and we had to generalise this condition to $Q^{2} \pi^{\text {torus }}=\pi^{\text {torus }}$. The same happens in the present case of the $A_{3} \times A_{1} \times B_{2}$-lattice. Let us for example consider the toroidal cycle $(1,0,0,0) \times(0,0,0,1) \times(1,0) \equiv \pi_{145}$ through the origin. Despite the fact that this three-cycle contains the $\mathbb{Z}_{2}$-invariant one-cycle $\pi_{4}$ and passes through a $\mathbb{Z}_{2^{-}}$ invariant point, $\pi_{145}$ is not $\mathbb{Z}_{2}$-invariant and therefore cannot be fractional. Indeed, one can check that $Q^{2} \pi_{145}=-\pi_{345}$. To impose the additional constraint on the numbers $\left(m^{i}, n^{i}, p^{i}, q^{i}\right)_{i=1,2} \times\left(m^{3}, n^{3}\right)$ ensuring the $Q^{2}$-invariance of the toroidal three-cycle we use the $Q$-action on the wrapping numbers

$$
\left(\begin{array}{c}
m^{i}, n^{i}, p^{i}, q^{i} \\
m^{3}, n^{3}
\end{array}\right) \stackrel{Q}{\longrightarrow}\left(\begin{array}{c}
-p^{i}, m^{i}-p^{i}, \quad n^{i}-p^{i},-q^{i} \\
m^{3}-n^{3}, 2 m^{3}-n^{3}
\end{array}\right) .
$$

For any toroidal three-cycle $\pi^{\text {torus }}$ of the form (2.26), it can be shown that the following statements are equivalent:

$$
\pi^{\text {torus }} \text { is } \mathbb{Z}_{2} \text {-invariant } \Longleftrightarrow Q^{2} \pi^{\text {torus }}=\pi^{\text {torus }} \Longleftrightarrow A_{1}+A_{3}=0 \text { and } B_{1}-B_{2}+B_{3}=0 .
$$

Only such $\mathbb{Z}_{2}$-invariant toroidal three-cycles can be used for the construction of the fractional cycles. From the factorisable case we know that the toroidal three-cycles giving rise to the fractional cycles have to contain $\mathbb{Z}_{2}$-invariant one-cycles. Indeed, it can be verified that any cycle which satisfies the conditions (2.34) can be written as

$$
\left(m^{1}, n^{1}, p^{1}, q^{1}\right) \times\left(m^{2}, n^{2}, p^{2}, q^{2}\right) \times\left(m^{3}, n^{3}\right)=\left(m^{1}, n^{1}, p^{1}, 0\right) \times\left(\tilde{p}^{2}, 0, \tilde{p}^{2}, \tilde{q}^{2}\right) \times\left(m^{3}, n^{3}\right)+\mathrm{R},
$$

where $\tilde{p}^{2}:=p^{2}-\frac{n^{2}}{n^{1}} p^{1}$ and $\tilde{q}^{2}:=q^{2}-\frac{n^{2}}{n^{1}} q^{1}$ for $n^{1} \neq 0 .^{2}$ The remaining term

$$
\mathrm{R}:=\left(0,0,0, q^{1}\right) \times\left(\tilde{p}^{2}, 0, \tilde{p}^{2}, \tilde{q}^{2}\right) \times\left(m^{3}, n^{3}\right)
$$

does not contribute to the bulk three-cycle and can be neglected. Therefore, any $\mathbb{Z}_{2^{-}}$ invariant three-cycle contains a linear combination of the $\mathbb{Z}_{2}$-invariant one-cycles $\pi_{1}+\pi_{3}$ and $\pi_{4}$.

The next step to construct the fractional cycles is to determine the exceptional part. In the factorisable case we identify which $\mathbb{Z}_{2}$-invariant points the toroidal three-cycle passes through. On the $A_{3} \times A_{1} \times B_{2}$-orbifold also the $\mathbb{Z}_{2}$-invariant lines are involved and so

\footnotetext{
${ }^{2} n^{1}=0$ gives rise to a similar result.
} 
we have to calculate their intersection with the toroidal three-cycle. Another important difference is that for the factorisable lattice $\left(T^{2}\right)^{3}$, the co-prime condition on the wrapping numbers $\left(n^{i}, m^{i}\right)$ per $T_{i}^{2}$ is necessary and sufficient for the fractional cycle to have a coprime decomposition in the unimodular basis. On the contrary, in the non-factorisable case the sufficiency condition gets lost and some further restrictions on the wrapping numbers $\left(m^{i}, n^{i}, p^{i}, q^{i}\right)$ are needed. It turns out that it is convenient to neglect these constraints at first and better to select the co-prime cycles after they are expressed in the unimodular basis. Thus, in this case, for the computation of the exceptional part we have to know not just which $\mathbb{Z}_{2}$-invariant lines the toroidal three-cycle intersects but also how many times. That requires some combinatorics.

Using the property (2.35) for the $\mathbb{Z}_{2}$-invariant three-cycles, we have only to compute how many times the cycle with the wrapping numbers $\left(m^{1}, n^{1}, p^{1}, 0\right)$ intersects the one-cycle $\pi_{1}+\pi_{3}$. We find that the number $N$ of intersection points is

$$
N=\text { g.c.d. }\left(m^{1}-p^{1}, n^{1}\right) \text {. }
$$

Furthermore, we make the following considerations and set several notations:

- The three-cycle can pass through both fixed lines 1 and 2 on the $A_{3}$-torus if and only if $\frac{m^{1}-p^{1}}{N}$ and $\frac{n^{1}}{N}$ are odd. We introduce three new parameters counting the different fixed lines traversed by the three-cycle

$$
\tau:= \begin{cases}2 & \text { if } \frac{m^{1}-p^{1}}{N} \text { and } \frac{n^{1}}{N} \text { are odd } \\ 1 & \text { otherwise }\end{cases}
$$

and

$$
\tau^{1}, \tau^{2} \in\{0,1\} \quad \text { with } \quad \tau^{1}+\tau^{2}=\tau .
$$

- On the $B_{2}$-torus, a one-cycle with even wrapping number $n^{3}$ passes through the fixed points $\overline{1}$ and $\overline{2}$ (both $\mathbb{Z}_{4}$-invariant), or $\overline{3}$ and $\overline{4}$ (both $\mathbb{Z}_{2}$-invariant). In this case, the fractional three-cycle contains either none or two exceptional three-cycles. If $n^{3}$ is odd, the one-cycle on $B_{2}$ intersects one $\mathbb{Z}_{4^{-}}$and one $\mathbb{Z}_{2}$-invariant fixed point, which in any case gives rise to one corresponding exceptional cycle. Thus, we define the parameter $\sigma$, which counts the number of exceptional three-cycles contributing to a fractional cycle for given bulk part, by

$$
\sigma:= \begin{cases}0,2 & \text { if } n^{3} \text { is even } \\ 1 & \text { if } n^{3} \text { is odd }\end{cases}
$$

- Furthermore, we introduce two parameters $s^{1}, s^{2}= \pm 1$ which describe the winding directions of the exceptional cycles.

Now we are able to write down the final form of the fractional three-cycle which is stemmed from the $\mathbb{Z}_{2}$-invariant toroidal three-cycle with the wrapping numbers $\left(m^{1}, n^{1}\right.$, $\left.p^{1}, 0\right) \times\left(\tilde{p}^{2}, 0, \tilde{p}^{2}, \tilde{q}^{2}\right) \times\left(m^{3}, n^{3}\right)$. It has the following form:

$$
\pi^{\text {frac }}=\frac{1}{2} \pi^{\text {bulk }}+N \frac{\tilde{p}^{2} \sigma}{2}\left(s^{1} \tau^{1} \gamma_{3}+s^{2} \tau^{2} \gamma_{4}\right)+N \frac{\tilde{q}^{2} \sigma}{2}\left(s^{1} \tau^{1} \bar{\gamma}_{3}+s^{2} \tau^{2} \bar{\gamma}_{4}\right) .
$$


It is easy to check that the coefficients of $\pi^{\text {bulk }}$, stemming from the toroidal cycle with the wrapping numbers $\left(m^{1}, n^{1}, p^{1}, 0\right) \times\left(\tilde{p}^{2}, 0, \tilde{p}^{2}, \tilde{q}^{2}\right) \times\left(m^{3}, n^{3}\right)$, have $N$ as defined in (2.36) as a common divisor too. Naively one can expect the restriction to $N=1$ for the co-prime condition. However, this is not necessarily true. Let us for example consider the toroidal cycle with wrapping numbers $(1,0,-1,0) \times(0,0,0,1) \times(0,1)$ passing through the fixed point 1 . It gives rise to the fractional cycle $\frac{1}{2}\left(2 \bar{\gamma}_{1} \pm 2 \bar{\gamma}_{3}\right)$. Although $N=2$ this cycle is co-prime because the cycle $\frac{1}{2}\left(\bar{\gamma}_{1} \pm \bar{\gamma}_{3}\right)$ does not exist due to the condition (2.34).

As a consequence of the considerations above we can summarise:

- Any fractional cycle containing only the one-cycle factor $\pi_{1}+\pi_{3}\left(\tilde{q}^{2}=0\right)$ within the exceptional part can be expressed as a sum over the unbarred cycles $\gamma_{i}$,

$$
\pi:=v^{1} \gamma_{1}+v^{2} \gamma_{2}+v^{3} \gamma_{3}+v^{4} \gamma_{4}
$$

with an even number of the coefficients $v^{i}$ with half-integer values.

- Any fractional cycle containing only the one-cycle factor $\pi_{4}\left(\tilde{p}^{2}=0\right)$ within the exceptional part can be expanded as a sum over the barred cycles $\bar{\gamma}_{i}$,

$$
\bar{\pi}:=\bar{v}^{1} \bar{\gamma}_{1}+\bar{v}^{2} \bar{\gamma}_{2}-\bar{v}^{3} \bar{\gamma}_{3}-\bar{v}^{4} \bar{\gamma}_{4}
$$

where $\bar{v}^{i}$ are either all half-integer or all integer.

In order to determine an integral symplectic basis for the three-cycles, we search for pairs of cycles $\pi$ and $\bar{\pi}$ such that $\pi \circ \bar{\pi}=-2$, with the coefficients $v^{i}$ and $\bar{v}^{i}$ satisfying $^{3}$ $\operatorname{sgn} v^{i}=\operatorname{sgn} \bar{v}^{i}$ for all $i$. All possible cycles can be combined in three sets:

1. $\left(v^{1}, v^{2}, v^{3}, v^{4}\right)=\left( \pm \frac{1}{2}, \pm \frac{1}{2}, 0,0\right)$ and $\left(\bar{v}^{1}, \bar{v}^{2}, \bar{v}^{3}, \bar{v}^{4}\right)=(\underline{ \pm 1, \pm 1,0,0})$ where the underlying denotes all permutations of entries.

2. $\left(v^{1}, v^{2}, v^{3}, v^{4}\right)=( \pm 1,0,0,0)$ for the (un-)barred cycles.

3. $\left(v^{1}, v^{2}, v^{3}, v^{4}\right)=\left( \pm \frac{1}{2}, \pm \frac{1}{2}, \pm \frac{1}{2}, \pm \frac{1}{2}\right)$ for the (un-)barred cycles.

Altogether there are 48 unbarred and 48 barred cycles. It is not difficult to specify a basis of these cycles. For the unbarred part we obtain

$$
\begin{aligned}
\alpha_{1} & :=\left(0, \frac{1}{2},-\frac{1}{2}, 0\right), \\
\alpha_{2} & :=\left(0,0, \frac{1}{2},-\frac{1}{2}\right), \\
\alpha_{3} & :=(0,0,0,1), \\
\alpha_{4} & :=\left(\frac{1}{2},-\frac{1}{2},-\frac{1}{2},-\frac{1}{2}\right),
\end{aligned}
$$

\footnotetext{
${ }^{3}$ The signum function is given by

$$
\operatorname{sgn}(x):= \begin{cases}-1 & \text { if } x<0 \\ 0 & \text { if } x=0 \\ 1 & \text { if } x>0\end{cases}
$$
}


and for the barred one,

$$
\begin{aligned}
& \bar{\alpha}_{1}:=(0,1,1,0), \\
& \bar{\alpha}_{2}:=(0,0,-1,1), \\
& \bar{\alpha}_{3}:=(0,0,0,-1), \\
& \bar{\alpha}_{4}:=\left(\frac{1}{2},-\frac{1}{2}, \frac{1}{2}, \frac{1}{2}\right) .
\end{aligned}
$$

Therefore, the fractional cycles form the $F_{4} \oplus F_{4}$-lattice, and the intersection matrix takes the form

$$
\alpha_{i} \circ \bar{\alpha}_{j}=\left(\begin{array}{cccc}
-2 & 1 & 0 & 0 \\
1 & -2 & 2 & 0 \\
0 & 1 & -2 & 1 \\
0 & 0 & 1 & -2
\end{array}\right) .
$$

This is the Cartan matrix for $F_{4}$, and it is unimodular. Indeed, it is easy to verify that any bulk, exceptional or fractional cycle can be expanded in the basis (2.43) and (2.44) with integer coefficients. In other words, since the determinant of the intersection matrix (2.45) is 1 , the $\alpha_{i}$ 's and $\bar{\alpha}_{i}$ 's form an integral basis of the homology lattice $H_{3}(M, \mathbb{Z})$.

\section{Intersecting brane worlds}

The aim of this article is not only the study of non-factorisable $\mathbb{Z}_{4}$-orbifolds but also model building with $\mathbb{Z}_{4}$-orientifolds of Type IIA superstring theory. We are interested in global supersymmetric models with a semi-realistic chiral spectrum, in particular Pati-Salammodels as D6-brane realisations with only three visible stacks, which have on other lattices been the most simple kind of global GUT model to be found, see e.g. [31, 34, 40, 46, 48, 8789]. The introduction of an anti-holomorphic involution $\mathcal{R}$ on the $\mathbb{Z}_{4}$-orbifold gives rise to orientifold six-planes (O6-planes), which wrap the fixed loci of $\mathcal{R} Q^{k}$ which together form some element of $H_{3}\left(T^{6} / \mathbb{Z}_{4}, \mathbb{Z}\right)$. In the following, we denote this homological cycle by $\pi_{\mathrm{O} 6}$. The $\left(\mathbb{Z}_{4}\right.$-orbits of $)$ O6-planes have negative RR charge, which has to be canceled by introducing stacks of $N_{a}$ space-time filling D6-branes which wrap a three-cycle $\pi_{a}$ on the orbifold. The RR tadpole cancellation condition is given by [86]

$$
\sum_{a} N_{a}\left(\pi_{a}+\pi_{a}^{\prime}\right)-4 \pi_{\mathrm{O} 6}=0
$$

where $\pi_{a}^{\prime}$ is the $\mathcal{R}$-image of the three-cycle $\pi_{a}$ with in general $\pi_{a}^{\prime} \neq \pi_{a}$. The resulting gauge group is then generically $\prod_{a} \mathrm{U}\left(N_{a}\right)$. The case $\pi_{a}^{\prime}=\pi_{a}$ gives rise to the rank-preserving symmetry enhancement: $\mathrm{U}\left(N_{a}\right) \hookrightarrow \mathrm{USp}\left(2 N_{a}\right)$ or $\mathrm{SO}\left(2 N_{a}\right)$. We call a model with the RR tadpole condition (3.1) implemented global, otherwise local. The chiral massless spectrum can be computed from topological intersection numbers [86]. For the gauge group $\prod_{a} \mathrm{U}\left(N_{a}\right)$ it is given in the table 2 .

Furthermore, the ( $\mathbb{Z}_{4}$-orbits of the) O6-planes preserve $\mathcal{N}=1$. For semi-realistic models to be supersymmetric, we have to require that all D6-branes preserve the same 


\begin{tabular}{|c|c|}
\hline \multicolumn{2}{|c|}{ Chiral spectrum } \\
\hline Representation & Multiplicity \\
\hline$(\mathbf{S y m})_{a}$ & $\frac{1}{2}\left(\pi_{a} \circ \pi_{a}^{\prime}-\pi_{a} \circ \pi_{\mathrm{O} 6}\right)$ \\
\hline$(\mathbf{A n t i})_{a}$ & $\frac{1}{2}\left(\pi_{a} \circ \pi_{a}^{\prime}+\pi_{a} \circ \pi_{\mathrm{O} 6}\right)$ \\
\hline$\left(\mathbf{N}_{a}, \overline{\mathbf{N}}_{b}\right)$ & $\pi_{a} \circ \pi_{b}$ \\
\hline$\left(\mathbf{N}_{a}, \mathbf{N}_{b}\right)$ & $\pi_{a} \circ \pi_{b}^{\prime}$ \\
\hline
\end{tabular}

Table 2. Chiral spectrum for intersecting D6-branes with gauge group $\prod_{a} \mathrm{U}\left(N_{a}\right)$.

supersymmetry, i.e., that they are wrapped on special Lagrangian three-cycles with the same calibration as the O6-planes. These additional constraints on the wrapped threecycles will be considered in more details in the section 3.2.

\subsection{Anti-holomorphic involutions $\mathcal{R}$ of $T^{6} / \mathbb{Z}_{4}$}

Before we start with the construction of the $\left(\mathbb{Z}_{4}\right.$-orbit of $)$ O6-plane(s), we have to calculate how the anti-holomorphic involution acts on the real lattice in each case. It is known [90, 91] that there exist a set of complex coordinates $\left\{z^{i}\right\}_{i=1,2,3}$ on which the twist $Q(2.23)$ acts diagonally

$$
Q^{t} z^{i}=e^{2 \pi i \zeta_{i}} z^{i}
$$

with the eigenvalues $(i,-1, i)$ for the shift vector $\vec{\zeta}=\frac{1}{4}(1,-2,1)$. In these coordinates, the anti-holomorphic involution (including $\mathbb{Z}_{4}$-twists) is simply given by complex conjugation

$$
\mathcal{R}\left(Q^{t}\right)^{n}: \quad z^{i} \rightarrow e^{i \theta_{n_{i}} \bar{z}^{i}}
$$

for some real parameter $\theta_{n_{i}}$. We will now discuss the a priori different possible choices of $\left(\theta_{n_{1}}, \theta_{n_{2}}, \theta_{n_{3}}\right)$ for each $\mathbb{Z}_{4}$-invariant background lattice and argue that some of the different choices lead to physically equivalent vacua, at least based on the allowed ranks of gauge groups in the RR tadpole cancellation conditions as well as on the counting of supersymmetric bulk cycles per given length.

\subsection{1 $\quad B_{2} \times\left(A_{1}\right)^{2} \times B_{2}$}

In the factorisable case, there exist a priori two choices of orientifold axes per two-torus [9294] denoted by $\mathbf{A}$ (reflection along the short one-cycle, here $\pi_{2 / 6}$ or some $\mathbb{Z}_{4}$-images thereof, cf. figure 1) and $\mathbf{B}$ (reflection along the long one-cycle, here $\pi_{1 / 5}$ ) for the $B_{2}$-tori and two lattice orientations $\mathbf{a}$ (rectangular lattice) and $\mathbf{b}$ (tilted lattice) for the $\left(A_{1}\right)^{2}$ torus. Due to the permutation symmetry $T_{1}^{2} \leftrightarrow T_{3}^{2}$, here the combinatorics provides at most six inequivalent choices of phases (3.3), denoted by $\mathbf{A a A}, \mathbf{A a B}, \mathbf{B a B}$ and $\mathbf{A b A}, \mathbf{A b B}, \mathbf{B b B}$, for which the O6-planes are displayed in table 3.

However, the massless closed string spectrum - encoded in the orientifold-even and -odd Hodge numbers $\left(h_{11}^{+}, h_{11}^{-}, h_{21}\right)$ counting vectors, Kähler and complex structure moduli as derived for generic Calabi-Yau backgrounds in [95] - , which was derived using CFT techniques in $[34,45]$ suggests that there are pairwise relations $\mathbf{A a A} \leftrightarrow \mathbf{B a B}$ and $\mathbf{A b A} \leftrightarrow$ 


\begin{tabular}{|c|c|c|c|}
\hline \multicolumn{3}{|c|}{ O6-planes on the $B_{2} \times\left(A_{1}\right)^{2} \times B_{2}$ lattice } \\
\hline lattice & $\mathbf{A a} / \mathbf{b A}$ & $\mathbf{A a} / \mathbf{b B}$ & $\mathbf{B a} / \mathbf{b B}$ \\
\hline $\mathcal{R}$ & $8(1-b) \pi_{236}$ & $4(1-b) \pi_{235}$ & $2(1-b) \pi_{135}$ \\
\hline $\mathcal{R} Q$ & $2\left(\pi_{1}+2 \pi_{2}\right) \wedge\left(\pi_{4}-b \pi_{3}\right) \wedge\left(\pi_{5}+2 \pi_{6}\right)$ & $4\left(\pi_{1}+2 \pi_{2}\right) \wedge\left(b \pi_{3}-\pi_{4}\right) \wedge \pi_{6}$ & $8 \pi_{2} \wedge\left(\pi_{4}-b \pi_{3}\right) \wedge \pi_{6}$ \\
\hline $\mathcal{R} Q^{2}$ & $-8(1-b)\left(\pi_{1}+\pi_{2}\right) \wedge \pi_{3} \wedge\left(\pi_{5}+\pi_{6}\right)$ & $4(1-b)\left(\pi_{1}+\pi_{2}\right) \wedge \pi_{3} \wedge\left(\pi_{5}+2 \pi_{6}\right)$ & $-2(1-b)\left(\pi_{1}+2 \pi_{2}\right) \wedge \pi_{3} \wedge\left(\pi_{5}+2 \pi_{6}\right)$ \\
\hline $\mathcal{R} Q^{3}$ & $2 \pi_{1} \wedge\left(b \pi_{3}-\pi_{4}\right) \wedge \pi_{5}$ & $4 \pi_{1} \wedge\left(\pi_{4}-b \pi_{3}\right) \wedge\left(\pi_{5}+\pi_{6}\right)$ & $8\left(\pi_{1}+\pi_{2}\right) \wedge\left(b \pi_{3}-\pi_{4}\right) \wedge\left(\pi_{5}+\pi_{6}\right)$ \\
\hline
\end{tabular}

Table 3. Fixed planes for the different lattice orientations of $B_{2} \times\left(A_{1}\right)^{2} \times B_{2}$, weighted with the number $N_{O 6}=2(1-b)$ of parallel O6-planes along $T_{2}^{2}$ with $b=0, \frac{1}{2}$ for $\mathbf{a}$ and $\mathbf{b}$, respectively.

$\mathbf{B b B}$, reducing the number of physically inequivalent backgrounds to four. This assumption is supported by the analogy to the relations among different lattice backgrounds derived for $\mathbb{Z}_{6}^{(\prime)}$ and $\mathbb{Z}_{2} \times \mathbb{Z}_{6}^{(\prime)}$ in $[42,46,48]$.

Since D6-branes on the factorisable $T^{6} /\left(\mathbb{Z}_{4} \times \Omega \mathcal{R}\right)$ orientifold have been considered at length in [34] with the result that at most two generations of chiral particles can be engineered by two supersymmetric intersecting D6-brane orbits, we will from now on concentrate on backgrounds with non-factorisable tori, where we will determine all a priori different choices of lattice orientations and then search for physical equivalences.

\subsection{2 $\quad A_{3} \times A_{3}$}

The eigenvectors of $Q^{t}$ with $Q$ defined in (2.12) give rise to the complex coordinates [84]

$$
\begin{aligned}
& z^{1}:=\frac{1}{\sqrt{2}}\left(x^{1}+i x^{2}-x^{3}\right) \\
& z^{2}:=\frac{1}{\sqrt{8 u_{2}}}\left(\left(x^{1}-x^{2}+x^{3}+\mathcal{U}\left(x^{4}-x^{5}+x^{6}\right)\right),\right. \\
& z^{3}:=\frac{1}{\sqrt{2}}\left(x^{4}+i x^{5}-x^{6}\right)
\end{aligned}
$$

where the complex structure is $\mathcal{U}:=u_{1}+i u_{2}=-\frac{R_{2}}{2 a R_{1}}\left(c+e+i \sqrt{-(c+e)^{2}+4 a b}\right)$.

With the transformation from the complex coordinates to the real ones, we are able to write down the action of $\mathcal{R}$ on the real lattice. There are a priori four possible choices of angles in the anti-holomorphic involution:

$$
\begin{aligned}
& \vec{\theta}=(0,0,0) \Rightarrow\left\{\begin{array}{ll}
\mathcal{R}_{1} e_{1}=e_{1}, & \mathcal{R}_{1} e_{2}=-e_{1}-e_{2}-e_{3}, \\
\mathcal{R}_{1} e_{3}=e_{3}, & \mathcal{R}_{1} e_{4}=u_{1}\left(e_{1}+e_{3}\right)-e_{6}, \\
\mathcal{R}_{1} e_{5}=-u_{1}\left(e_{1}+e_{3}\right)-e_{5}, & \mathcal{R}_{1} e_{6}=u_{1}\left(e_{1}+e_{3}\right)-e_{4},
\end{array}\right\} \quad \text { AAA } \\
& \vec{\theta}=\left(\frac{\pi}{2}, 0,0\right) \Rightarrow\left\{\begin{array}{ll}
\mathcal{R}_{2} e_{1}=e_{1}+e_{2}+e_{3}, & \mathcal{R}_{2} e_{2}=-e_{3}, \\
\mathcal{R}_{2} e_{3}=-e_{2}, & \mathcal{R}_{1} e_{4}=u_{1}\left(e_{1}+e_{3}\right)-e_{6}, \\
\mathcal{R}_{1} e_{5}=-u_{1}\left(e_{1}+e_{3}\right)-e_{5}, & \mathcal{R}_{1} e_{6}=u_{1}\left(e_{1}+e_{3}\right)-e_{4},
\end{array}\right\} \quad \text { BAA } \\
& \overrightarrow{\mathcal{R}_{3} e_{2}=-e_{1}-e_{2}-e_{3},} \\
& \vec{\theta}=\left(0,0, \frac{\pi}{2}\right) \Rightarrow\left\{\begin{array}{ll}
\mathcal{R}_{3} e_{1}=e_{1}, & \mathcal{R}_{3} e_{4}=u_{1}\left(e_{1}+e_{3}\right)+e_{5}, \\
\mathcal{R}_{3} e_{3}=e_{3}, & \mathcal{R}_{3} e_{6}=u_{1}\left(e_{1}+e_{3}\right)-e_{4}-e_{5}-e_{6},
\end{array}\right\}
\end{aligned}
$$




\begin{tabular}{|c|c|}
\hline \multicolumn{2}{|c|}{ O6-planes for AAA-lattice of $A_{3} \times A_{3}$-orbifold } \\
\hline Projection & Fixed point set \\
\hline $\mathcal{R}_{1}$ & $2\left(\pi_{134}-\pi_{136}\right)$ \\
\hline $\mathcal{R}_{1} Q$ & $2\left(\pi_{245}+\pi_{246}-\pi_{256}+\pi_{345}+\pi_{346}-\pi_{356}\right)$ \\
\hline $\mathcal{R}_{1} Q^{2}$ & $2\left(\pi_{124}+2 \pi_{125}+\pi_{126}-\pi_{234}-2 \pi_{235}-\pi_{236}\right)$ \\
\hline $\mathcal{R}_{1} Q^{3}$ & $2\left(-\pi_{145}+\pi_{146}+\pi_{156}-\pi_{245}+\pi_{246}+\pi_{256}\right)$ \\
\hline
\end{tabular}

Table 4. Toroidal cycles wrapped by the O6-planes on the AAA lattice orientation of $A_{3} \times A_{3}$.

$$
\vec{\theta}=\left(\frac{\pi}{2}, 0, \frac{\pi}{2}\right) \Rightarrow\left\{\begin{array}{ll}
\mathcal{R}_{4} e_{1}=e_{1}+e_{2}+e_{3}, & \mathcal{R}_{4} e_{2}=-e_{3}, \\
\mathcal{R}_{4} e_{3}=-e_{2}, & \mathcal{R}_{4} e_{4}=u_{1}\left(e_{1}+e_{3}\right)+e_{5}, \\
\mathcal{R}_{4} e_{5}=u_{1}\left(e_{1}+e_{3}\right)+e_{4}, & \mathcal{R}_{4} e_{6}=u_{1}\left(e_{1}+e_{3}\right)-e_{4}-e_{5}-e_{6} .
\end{array}\right\} \quad \mathbf{B A B}
$$

Here we took the notation for the lattices from [63], which we will now explain. The complex coordinates (3.4) parametrise the three-planes ${ }^{4}$ where $Q$ acts as a rotation. A means that, in their choice of basis, the orientifold plane lies along the horizontal axis in the corresponding plane, while $\mathbf{B}$ corresponds to the angle of the orientifold plane with respect to the horizontal axis being $\pi / 4$.

Note also that in contrast to the $B_{2} \times\left(A_{1}\right)^{2} \times B_{2}$-orbifold, the BAA- and AAB-lattices are really different geometrically and cannot be related by exchanging $T_{1}^{3} \leftrightarrow T_{2}^{3}$. This will be further specified in the sections 3.3 and 4 .

Since only a crystallographic action on the lattice is allowed $\left(\mathcal{R}_{i} r=r+\Lambda\right.$ for arbitrary lattice vectors $r \in \Lambda$ ), the real part of the complex structure can take only the value $u_{1}=0$ ( $\Rightarrow c+e=0$ in (2.13) and only $\mathbf{A}$ as middle entry of the lattice orientation). The orientifold projections act then on the homology classes of three-cycles (2.15) in the following way:

$$
\begin{array}{ccc}
\mathcal{R}_{1}: & \gamma_{1} \leftrightarrow-\gamma_{2}, & \bar{\gamma}_{1} \leftrightarrow \bar{\gamma}_{2} . \\
\mathcal{R}_{2 / 3}: & \begin{cases}\gamma_{1} \rightarrow \gamma_{1}, & \bar{\gamma}_{1} \rightarrow-\bar{\gamma}_{1}, \\
\gamma_{2} \rightarrow-\gamma_{2}, & \bar{\gamma}_{2} \rightarrow \bar{\gamma}_{2} .\end{cases} \\
\mathcal{R}_{4}: & \gamma_{1} \leftrightarrow \gamma_{2}, & \bar{\gamma}_{1} \leftrightarrow-\bar{\gamma}_{2} .
\end{array}
$$

Furthermore, we calculate the fixed point set for the orientifold involutions $\left\{\mathcal{R}_{i}, \mathcal{R}_{i} Q\right.$, $\left.\mathcal{R}_{i} Q^{2}, \mathcal{R}_{i} Q^{3}\right\}$. The results are listed in tables $4-7$ for the respective $\mathcal{R}_{i}$.

The fixed point set under $\mathcal{R}_{i} Q^{n}$ is computed in the following way, e.g. for $\mathcal{R}_{1}$ : first, we calculate the three eigenvectors of $\mathcal{R}_{1}$ corresponding to the eigenvalue 1 , in this case $e_{1}, e_{3}$ and $e_{4}-e_{6}$. The to these vectors corresponding one-cycles $\pi_{i}$ span a three-cycle $\pi_{134}-\pi_{136}$. The next step is to determine how many three-cycles of the same homology class we have which are point-wise $\mathcal{R}_{1}$-invariant. In the case at hand, we obtain two such submanifolds, which go through the $\mathcal{R}_{1}$ fixed points $\left(0,0,0,0, \frac{m}{2}, 0\right)$ with $m \in\{0,1\}$. The projection $\mathcal{R}_{1} Q$ leads to similar results. The fixed point sets of $\mathcal{R} Q^{2}$ and $\mathcal{R} Q^{3}$ can be calculated by acting

\footnotetext{
${ }^{4}$ These three-planes are closely related to the "factorisation" of the non-factorisable torus we will discuss in the next section.
} 


\begin{tabular}{|c|c|}
\hline \multicolumn{2}{|c|}{ O6-planes for BAA-lattice of $A_{3} \times A_{3}$-orbifold } \\
\hline Projection & Fixed point set \\
\hline $\mathcal{R}_{2}$ & $2\left(-\pi_{124}+\pi_{126}+\pi_{134}-\pi_{136}+\pi_{234}-\pi_{236}\right)$ \\
\hline $\mathcal{R}_{2} Q$ & $2\left(-\pi_{145}-\pi_{146}+\pi_{156}+\pi_{345}+\pi_{346}-\pi_{356}\right)$ \\
\hline $\mathcal{R}_{2} Q^{2}$ & $2\left(\pi_{124}+2 \pi_{125}+\pi_{126}+\pi_{134}+2 \pi_{135}+\pi_{136}-\pi_{234}-2 \pi_{235}-\pi_{236}\right)$ \\
\hline $\mathcal{R}_{2} Q^{3}$ & $2\left(-\pi_{145}+\pi_{146}+\pi_{156}-2 \pi_{245}+2 \pi_{246}+2 \pi_{256}-\pi_{345}+\pi_{346}+\pi_{356}\right)$ \\
\hline
\end{tabular}

Table 5. Toroidal cycles wrapped by the O6-planes for the BAA lattice orientation of $A_{3} \times A_{3}$.

\begin{tabular}{|c|c|}
\hline \multicolumn{2}{|c|}{ O6-planes for $A$ AB-lattice of $A_{3} \times A_{3}$-orbifold } \\
\hline Projection & Fixed point set \\
\hline $\mathcal{R}_{3}$ & $2\left(\pi_{134}+\pi_{135}\right)$ \\
\hline $\mathcal{R}_{3} Q$ & $2\left(\pi_{246}+\pi_{346}\right)$ \\
\hline $\mathcal{R}_{3} Q^{2}$ & $2\left(\pi_{125}+\pi_{126}-\pi_{235}-\pi_{236}\right)$ \\
\hline $\mathcal{R}_{3} Q^{3}$ & $2\left(-\pi_{145}+\pi_{156}-\pi_{245}+\pi_{256}\right)$ \\
\hline
\end{tabular}

Table 6. Toroidal cycles wrapped by the O6-planes for the AAB lattice orientation of $A_{3} \times A_{3}$.

\begin{tabular}{|c|c|}
\hline \multicolumn{2}{|c|}{ O6-planes for BAB-lattice of $A_{3} \times A_{3}$-orbifold } \\
\hline Projection & Fixed point set \\
\hline $\mathcal{R}_{4}$ & $2\left(-\pi_{124}-\pi_{125}+\pi_{134}+\pi_{135}+\pi_{234}+\pi_{235}\right)$ \\
\hline $\mathcal{R}_{4} Q$ & $2\left(\pi_{146}-\pi_{346}\right)$ \\
\hline $\mathcal{R}_{4} Q^{2}$ & $2\left(\pi_{125}+\pi_{126}+\pi_{135}+\pi_{136}-\pi_{235}-\pi_{236}\right)$ \\
\hline $\mathcal{R}_{4} Q^{3}$ & $2\left(\pi_{145}-\pi_{156}+2 \pi_{245}-2 \pi_{256}+\pi_{345}-\pi_{356}\right)$ \\
\hline
\end{tabular}

Table 7. Toroidal cycles wrapped by the O6-planes for the BAB lattice orientation of $A_{3} \times A_{3}$.

with $Q$ on the $\mathcal{R}$ - and $\mathcal{R} Q$-fixed point sets, respectively. After all fixed point sets have been computed, from purely geometric considerations there still remains an ambiguity in both the global sign of all sets and the relative sign between the $\mathcal{R}$ - and $\mathcal{R} Q$-sets which needs to be fixed, i.e., the three-cycle $\pm\left(\operatorname{Fix}\left(\mathcal{R}+\mathcal{R} Q^{2}\right) \pm \operatorname{Fix}\left(\mathcal{R} Q+\mathcal{R} Q^{3}\right)\right)$ is invariant for any choice of the signs. Each of the two sets of fixed points corresponds to a different bulk cycle. Asking both of them to have the same calibration will fix the relative sign between them. The global sign is fixed once we choose one of the two possible calibration conditions (either $\int\left(e^{i \varphi} \Omega_{3}\right)>0$ or $\int\left(e^{i \varphi+i \pi} \Omega_{3}\right)>0$ ); alternatively, choosing the global sign will fix the calibration condition that has to be used. This calibration will be explained in more detail in section 3.2 . 
Adding all contributions, we can express the corresponding O6-planes as elements of $H_{3}\left(T^{6} / \mathbb{Z}_{4}, \mathbb{Z}\right)$ :

$$
\begin{aligned}
& \pi_{\mathrm{O} 6_{1}}:=2\left(\gamma_{2}-\gamma_{1}\right)-2\left(\bar{\gamma}_{1}+\bar{\gamma}_{2}\right), \\
& \pi_{\mathrm{O} 6_{2}}:=-4 \gamma_{1}-4 \bar{\gamma}_{2}, \\
& \pi_{\mathrm{O} 6_{3}}:=-2 \gamma_{1}-2 \bar{\gamma}_{2}, \\
& \pi_{\mathrm{O} 6_{4}}:=2\left(\gamma_{1}+\gamma_{2}\right)+2\left(\bar{\gamma}_{2}-\bar{\gamma}_{1}\right) .
\end{aligned}
$$

Obviously, the O6-planes (3.12) are invariant under the corresponding orientifold projections given in equations (3.9), (3.10) and (3.11).

\subsection{3 $\quad A_{3} \times A_{1} \times B_{2}$}

We can approach the $A_{3} \times A_{1} \times B_{2}$-case in an analogous way. Due to (3.2), the complex coordinates are:

$$
\begin{aligned}
& z^{1}=\frac{1}{\sqrt{2}}\left(x^{1}+i x^{2}-x^{3}\right) \\
& z^{2}=\frac{1}{2 \sqrt{2 \operatorname{Im}(\mathcal{U})}}\left(x^{1}-x^{2}+x^{3}+2 \mathcal{U} x^{4}\right) \\
& z^{3}=x^{5}-\frac{x^{6}}{2}+i \frac{x^{6}}{2}
\end{aligned}
$$

with the complex structure

$$
\mathcal{U}:=u_{1}+i u_{2}:=-\frac{R_{1}}{2 a R_{3}}\left(d+i \sqrt{-a-d^{2}}\right) .
$$

For this non-factorisable lattice, there are four possible orientations:

$$
\begin{aligned}
& \vec{\theta}=(0,0,0) \Rightarrow\left\{\begin{array}{ll}
\mathcal{R}_{1} e_{1}=e_{1}, & \mathcal{R}_{1} e_{2}=-e_{1}-e_{2}-e_{3}, \\
\mathcal{R}_{1} e_{3}=e_{3}, & \mathcal{R}_{1} e_{4}=2 u_{1}\left(e_{1}+e_{3}\right)-e_{4}, \\
\mathcal{R}_{1} e_{5}=e_{5}, & \mathcal{R}_{1} e_{6}=-e_{5}-e_{6},
\end{array}\right\} \quad \mathbf{A A B} \\
& \vec{\theta}=\left(0,0,-\frac{\pi}{2}\right) \Rightarrow\left\{\begin{array}{ll}
\mathcal{R}_{2} e_{1}=e_{1}, & \mathcal{R}_{2} e_{2}=-e_{1}-e_{2}-e_{3}, \\
\mathcal{R}_{2} e_{3}=e_{3}, & \mathcal{R}_{2} e_{4}=2 u_{1}\left(e_{1}+e_{3}\right)-e_{4}, \\
\mathcal{R}_{2} e_{5}=-e_{5}-2 e_{6}, & \mathcal{R}_{2} e_{6}=e_{6},
\end{array}\right\} \quad \text { AAA } \\
& \vec{\theta}=\left(-\frac{\pi}{2}, 0,-\frac{\pi}{2}\right) \Rightarrow\left\{\begin{array}{ll}
\mathcal{R}_{3} e_{1}=-e_{2}, & \mathcal{R}_{3} e_{2}=-e_{1}, \\
\mathcal{R}_{3} e_{3}=e_{1}+e_{2}+e_{3}, & \mathcal{R}_{3} e_{4}=2 u_{1}\left(e_{1}+e_{3}\right)-e_{4}, \\
\mathcal{R}_{3} e_{5}=-e_{5}-2 e_{6}, & \mathcal{R}_{3} e_{6}=e_{6}
\end{array}\right\}
\end{aligned}
$$

and

$$
\vec{\theta}=\left(-\frac{\pi}{2}, 0,0\right) \Rightarrow\left\{\begin{array}{ll}
\mathcal{R}_{4} e_{1}=-e_{2}, & \mathcal{R}_{4} e_{2}=-e_{1} \\
\mathcal{R}_{4} e_{3}=e_{1}+e_{2}+e_{3}, & \mathcal{R}_{4} e_{4}=2 u_{1}\left(e_{1}+e_{3}\right)-e_{4}, \\
\mathcal{R}_{4} e_{5}=e_{5}, & \mathcal{R}_{4} e_{6}=-e_{5}-e_{6} .
\end{array}\right\}
$$

Here we also took again the notation for the lattices from [63]. Since only the crystallographic action on the lattice is allowed $\left(\mathcal{R}_{i} r=r+\Lambda\right.$ for arbitrary lattice vectors $\left.r \in \Lambda\right)$, the real part of the complex structure can take only two values $u_{1}=0, \frac{1}{2}$. We adapt the above lattice notation for different values of $u_{1}$ in the following way: we write a small $\mathbf{a}$ 


\begin{tabular}{|c|c|}
\hline \multicolumn{2}{|c|}{ O6-planes for AAB-lattice of $A_{3} \times A_{1} \times B_{2}$-orbifold } \\
\hline Projection & Fixed point set \\
\hline $\mathcal{R}_{1}$ & $2 \pi_{135}$ \\
\hline $\mathcal{R}_{1} Q$ & $4\left(u_{1}\left(\pi_{126}+\pi_{136}-\pi_{236}\right)+\pi_{246}+\pi_{346}\right)$ \\
\hline $\mathcal{R}_{1} Q^{2}$ & $2 \pi_{125}+4 \pi_{126}-2 \pi_{235}-4 \pi_{236}$ \\
\hline $\mathcal{R}_{1} Q^{3}$ & $4\left(u_{1}\left(\pi_{135}+\pi_{136}-\pi_{125}-\pi_{126}+\pi_{235}+\pi_{236}\right)-\pi_{145}-\pi_{146}-\pi_{245}-\pi_{246}\right)$ \\
\hline
\end{tabular}

Table 8. Toroidal cycles wrapped by the O6-planes for the $\mathbf{A A B}$ orientation of $A_{3} \times A_{1} \times B_{2}$.

and $\mathbf{b}$ as a subscript after the first $\mathbf{A}$ for $u_{1}=0$ and $u_{1}=\frac{1}{2}$, respectively. The $\mathbf{b}$-type lattice is to our best knowledge investigated here for the first time, while the D6-brane configurations, which cancel the bulk RR tadpoles locally on top of the O6-planes, in [63] correspond to the a-type choice $u_{1}=0$.

The orientifold action on the homological three-cycles is given by

$$
\begin{aligned}
& \mathcal{R}_{1}: \quad \begin{cases}\gamma_{1} \rightarrow \gamma_{2}, & \bar{\gamma}_{1} \rightarrow-\bar{\gamma}_{2}-2 u_{1} \gamma_{2}, \\
\gamma_{2} \rightarrow \gamma_{1}, & \bar{\gamma}_{2} \rightarrow-\bar{\gamma}_{1}-2 u_{1} \gamma_{1}, \\
\gamma_{3} \rightarrow \gamma_{3}, & \bar{\gamma}_{3} \rightarrow-\bar{\gamma}_{3}+2 u_{1} \gamma_{3}, \\
\gamma_{4} \rightarrow \gamma_{4}, & \bar{\gamma}_{4} \rightarrow-\bar{\gamma}_{4}+2 u_{1} \gamma_{4} .\end{cases} \\
& \mathcal{R}_{2}: \quad \begin{cases}\gamma_{1} \rightarrow \gamma_{1}, & \bar{\gamma}_{1} \rightarrow-\bar{\gamma}_{1}-2 u_{1} \gamma_{1}, \\
\gamma_{2} \rightarrow-\gamma_{2}, & \bar{\gamma}_{2} \rightarrow \bar{\gamma}_{2}+2 u_{1} \gamma_{2}, \\
\gamma_{3} \rightarrow-\gamma_{3}, & \bar{\gamma}_{3} \rightarrow \bar{\gamma}_{3}-2 u_{1} \gamma_{3}, \\
\gamma_{4} \rightarrow-\gamma_{4}, & \bar{\gamma}_{4} \rightarrow \bar{\gamma}_{4}-2 u_{1} \gamma_{4} .\end{cases} \\
& \mathcal{R}_{3}: \quad \begin{cases}\gamma_{1} \rightarrow-\gamma_{2}, & \bar{\gamma}_{1} \rightarrow \bar{\gamma}_{2}+2 u_{1} \gamma_{2}, \\
\gamma_{2} \rightarrow-\gamma_{1}, & \bar{\gamma}_{2} \rightarrow \bar{\gamma}_{1}+2 u_{1} \gamma_{1}, \\
\gamma_{3} \rightarrow-\gamma_{3}, & \bar{\gamma}_{3} \rightarrow \bar{\gamma}_{3}-2 u_{1} \gamma_{3}, \\
\gamma_{4} \rightarrow-\gamma_{4}, & \bar{\gamma}_{4} \rightarrow \bar{\gamma}_{4}-2 u_{1} \gamma_{4} .\end{cases} \\
& \mathcal{R}_{4}: \quad \begin{cases}\gamma_{1} \rightarrow \gamma_{1}, & \bar{\gamma}_{1} \rightarrow-\bar{\gamma}_{1}-2 u_{1} \gamma_{1}, \\
\gamma_{2} \rightarrow-\gamma_{2}, & \bar{\gamma}_{2} \rightarrow \bar{\gamma}_{2}+2 u_{1} \gamma_{2}, \\
\gamma_{3} \rightarrow \gamma_{3}, & \bar{\gamma}_{3} \rightarrow-\bar{\gamma}_{3}+2 u_{1} \gamma_{3}, \\
\gamma_{4} \rightarrow \gamma_{4}, & \bar{\gamma}_{4} \rightarrow-\bar{\gamma}_{4}+2 u_{1} \gamma_{4} .\end{cases}
\end{aligned}
$$

Furthermore, we can calculate the fixed point sets for the orientifold projections $\left\{\mathcal{R}_{i}, \mathcal{R}_{i} Q\right.$, $\left.\mathcal{R}_{i} Q^{2}, \mathcal{R}_{i} Q^{3}\right\}$. The results are listed in tables $8-11$ for the respective $\mathcal{R}_{i}$. In figures $4-8$ we illustrate the O6-planes for the lattices $\mathbf{A}_{\mathbf{a}} \mathbf{A B}$ and $\mathbf{A}_{\mathbf{a}} \mathbf{B B} .^{5}$

\footnotetext{
${ }^{5}$ We will see in the next section that the other a-type lattices are related to these.
} 


\begin{tabular}{|c|c|}
\hline \multicolumn{2}{|c|}{ O6-planes for AAA-lattice of $A_{3} \times A_{1} \times B_{2}$-orbifold } \\
\hline Projection & Fixed point set \\
\hline $\mathcal{R}_{2}$ & $4 \pi_{136}$ \\
\hline $\mathcal{R}_{2} Q$ & $2\left(-u_{1}\left(\pi_{125}+\pi_{135}-\pi_{235}+2\left(\pi_{126}+\pi_{136}-\pi_{236}\right)\right)-\pi_{245}-\pi_{345}-2\left(\pi_{246}+\pi_{346}\right)\right)$ \\
\hline $\mathcal{R}_{2} Q^{2}$ & $4\left(-\pi_{125}-\pi_{126}+\pi_{235}+\pi_{236}\right)$ \\
\hline $\mathcal{R}_{2} Q^{3}$ & $2\left(-u_{1}\left(\pi_{135}-\pi_{125}+\pi_{235}\right)-\pi_{145}-\pi_{245}\right)$ \\
\hline
\end{tabular}

Table 9. Toroidal cycles wrapped by the O6-planes for the AAA orientation of $A_{3} \times A_{1} \times B_{2}$.

\begin{tabular}{|c|c|}
\hline \multicolumn{2}{|c|}{ O6-planes for ABA-lattice of $A_{3} \times A_{1} \times B_{2}$-orbifold } \\
\hline Projection & Fixed point set \\
\hline $\mathcal{R}_{3}$ & $\left(4-4 u_{1}\right)\left(\pi_{126}+\pi_{136}-\pi_{236}\right)$ \\
\hline $\mathcal{R}_{3} Q$ & $2\left(-2 u_{1}\left(\pi_{125}-\pi_{235}+2 \pi_{126}-2 \pi_{236}\right)-\pi_{145}-2 \pi_{245}-\pi_{345}-2\left(\pi_{146}+2 \pi_{246}+\pi_{346}\right)\right)$ \\
\hline $\mathcal{R}_{3} Q^{2}$ & $\left(4-4 u_{1}\right)\left(-\pi_{125}-\pi_{126}+\pi_{135}+\pi_{136}+\pi_{235}+\pi_{236}\right)$ \\
\hline $\mathcal{R}_{3} Q^{3}$ & $2\left(-2 u_{1} \pi_{135}+\pi_{145}-\pi_{345}\right)$ \\
\hline
\end{tabular}

Table 10. Toroidal cycles wrapped by the O6-planes for the $\mathbf{A B A}$ orientation of $A_{3} \times A_{1} \times B_{2}$.

\begin{tabular}{|c|c|}
\hline \multicolumn{2}{|c|}{ O6-planes for ABB-lattice of $A_{3} \times A_{1} \times B_{2}$-orbifold } \\
\hline Projection & Fixed point set \\
\hline $\mathcal{R}_{4}$ & $\left(2-2 u_{1}\right)\left(\pi_{125}+\pi_{135}-\pi_{235}\right)$ \\
\hline $\mathcal{R}_{4} Q$ & $4\left(2 u_{1}\left(\pi_{126}-\pi_{236}\right)+\pi_{146}+2 \pi_{246}+\pi_{346}\right)$ \\
\hline $\mathcal{R}_{4} Q^{2}$ & $\left(2-2 u_{1}\right)\left(\pi_{125}+2 \pi_{126}-\pi_{135}-2 \pi_{136}-\pi_{235}-2 \pi_{236}\right)$ \\
\hline $\mathcal{R}_{4} Q^{3}$ & $4\left(2 u_{1}\left(\pi_{135}+\pi_{136}\right)-\pi_{145}-\pi_{146}+\pi_{345}+\pi_{346}\right)$ \\
\hline
\end{tabular}

Table 11. Toroidal cycles wrapped by the O6-planes for the $\mathbf{A B B}$ orientation of $A_{3} \times A_{1} \times B_{2}$.

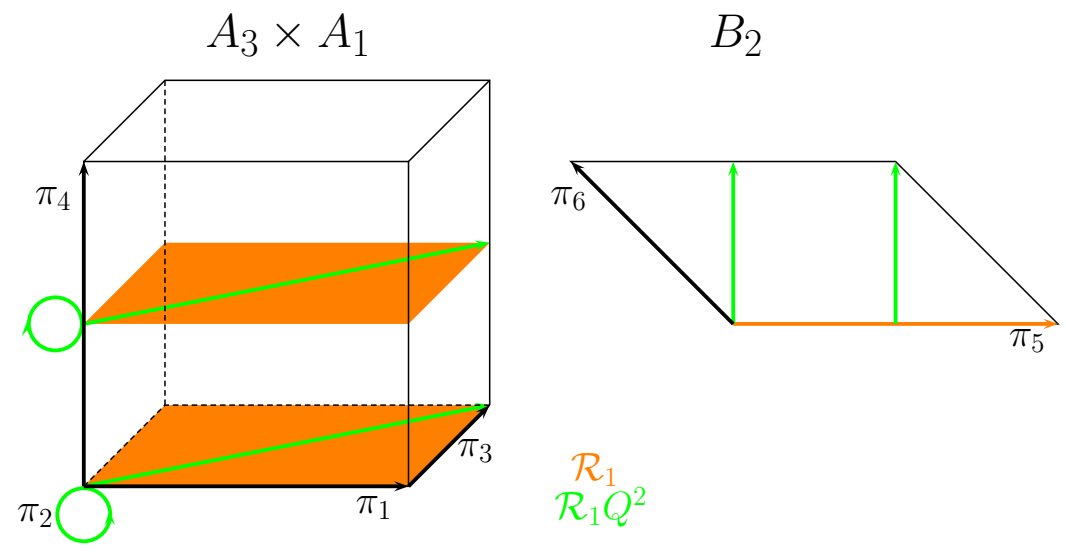

Figure 4. $\mathcal{R}_{1^{-}}$and $\mathcal{R}_{1} Q^{2}$-contributions to the O6-planes of the $A_{3} \times A_{1} \times B_{2}$-orientifold with A AB-lattice $\left(u_{1}=0\right)$. 


$$
A_{3} \times A_{1}
$$

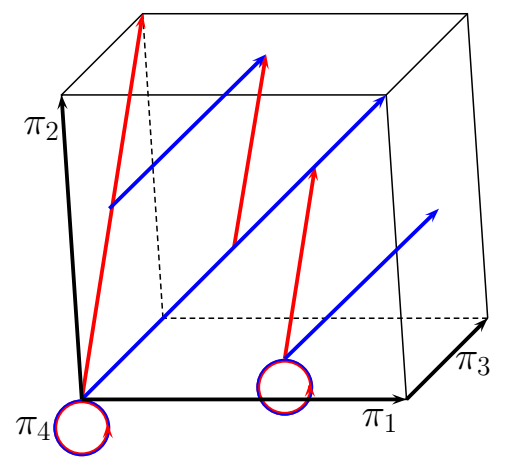

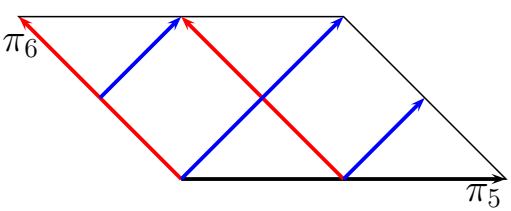

$\mathcal{R}_{1} Q$
$\mathcal{R}_{1} Q^{3}$

Figure 5. $\mathcal{R}_{1} Q$ - and $\mathcal{R}_{1} Q^{3}$-contributions to the O6-plane of the $A_{3} \times A_{1} \times B_{2}$-orientifold with $\mathbf{A}_{\mathbf{a}}$ AB-lattice $\left(u_{1}=0\right)$.

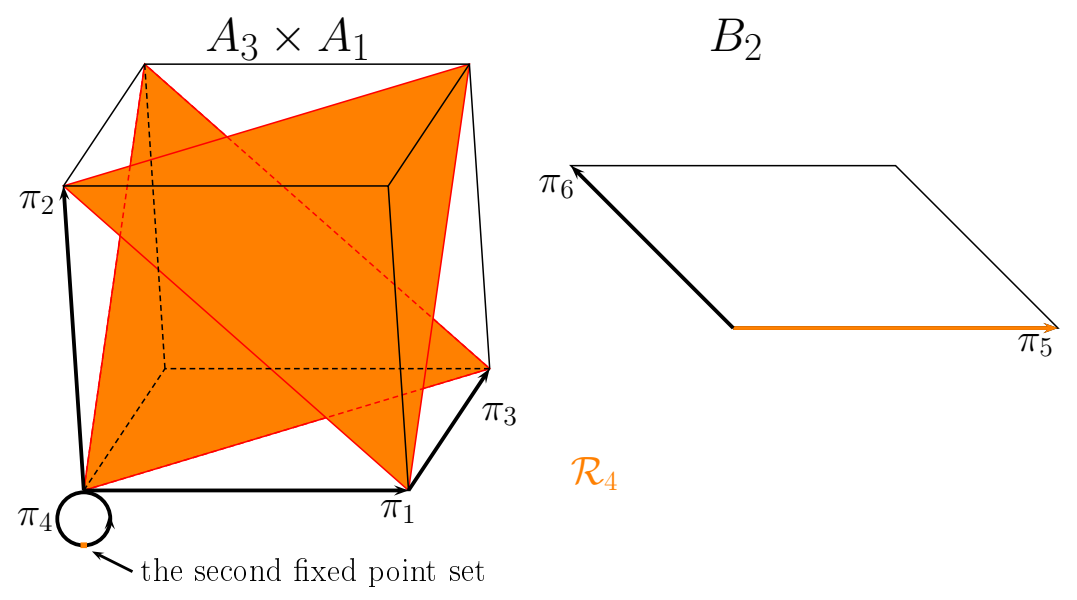

Figure 6. $\mathcal{R}_{4}$-contribution to the O6-plane of the $A_{3} \times A_{1} \times B_{2}$-orientifold with $\mathbf{A}_{\mathbf{a}} \mathbf{B B}$-lattice $\left(u_{1}=0\right)$. The second fixed point set is located at $\pi_{4}=\frac{1}{2}$ and denoted by the orange point in the picture.

Adding up all contributions we obtain the corresponding O6-planes:

$$
\begin{aligned}
& \pi_{\mathrm{O} 6_{1}}:=\left(1-2 u_{1}\right) \gamma_{1}+\left(1+2 u_{1}\right) \gamma_{2}-2\left(\bar{\gamma}_{1}-\bar{\gamma}_{2}\right), \\
& \pi_{\mathrm{O}_{2}}:=-2 \gamma_{1}-2 u_{1} \gamma_{2}-2 \bar{\gamma}_{2} \\
& \pi_{\mathrm{O} 6_{3}}:=2\left(\gamma_{2}-\gamma_{1}\right)-4 u_{1} \gamma_{2}-2\left(\bar{\gamma}_{1}+\bar{\gamma}_{2}\right) \\
& \pi_{\mathrm{O} 6_{4}}:=\left(2-2 u_{1}\right) \gamma_{1}+4 u_{1} \gamma_{2}+4 \bar{\gamma}_{2} .
\end{aligned}
$$

It is easy to check that the resulting O6-planes are invariant under corresponding orientifold projections defined in equations (3.19) to (3.22).

\subsection{Supersymmetric cycles}

From the phenomenological point of view (like chirality and stability at low energies) $\mathcal{N}=1$ supersymmetric models are particularly interesting. This requires the D6-branes 


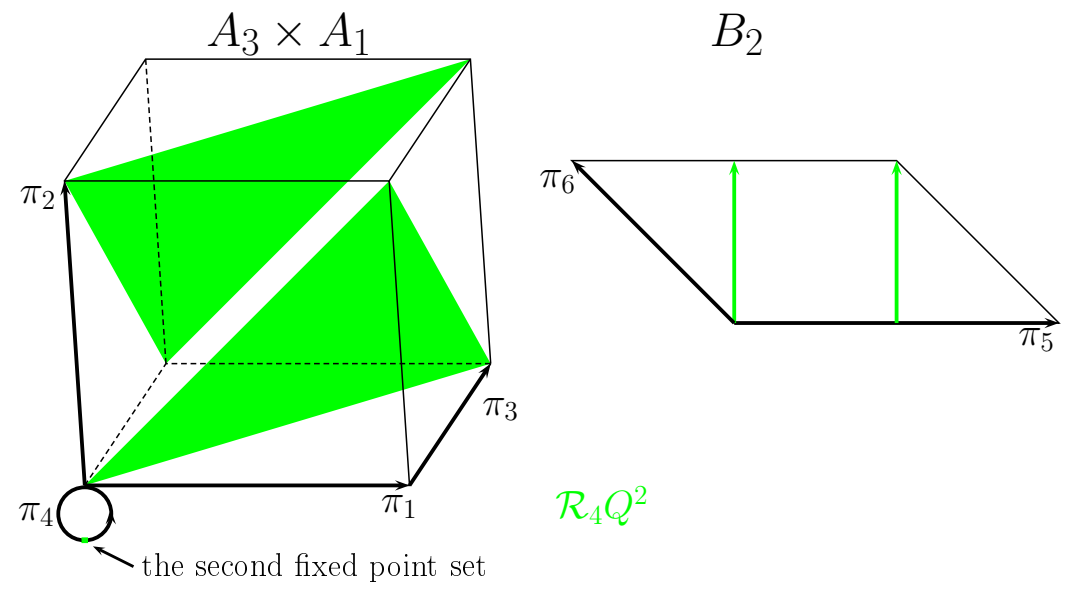

Figure 7. $\mathcal{R}_{4} Q^{2}$-contributions to the O6-plane of the $A_{3} \times A_{1} \times B_{2}$-orientifold with $\mathbf{A}_{\mathbf{a}} \mathbf{B B}$-lattice $\left(u_{1}=0\right)$. The second fixed point set is located at $\pi_{4}=\frac{1}{2}$ and denoted by the green point in the picture.

$$
A_{3} \times A_{1}
$$

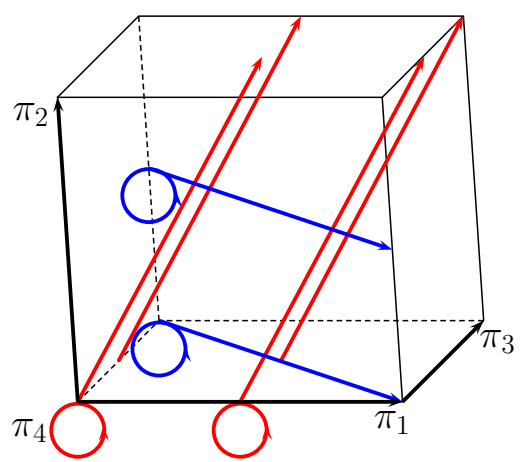

$B_{2}$

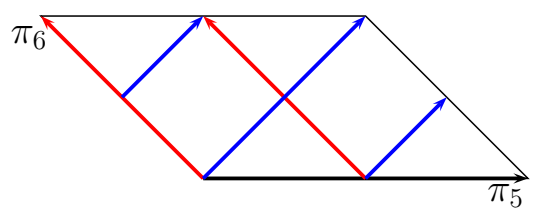

$\mathcal{R} Q$

Figure 8. $\mathcal{R}_{4} Q$ - and $\mathcal{R}_{4} Q^{3}$-contributions to the O6-plane of the $A_{3} \times A_{1} \times B_{2}$-orientifold with A $\mathbf{a B}$-lattice $\left(u_{1}=0\right)$.

to preserve some supersymmetry, which leads to additional geometrical conditions on the allowed three-cycles; namely, the three-cycles have to be special Lagrangian. A three-cycle $\pi$ is called special Lagrangian $(s L a g)$ if it satisfies

$$
\begin{array}{r}
\left.J\right|_{\pi}=0 \\
\left.\operatorname{Im}\left(e^{i \varphi} \Omega_{3}\right)\right|_{\pi}=0 \\
\left.\operatorname{Re}\left(e^{i \varphi} \Omega_{3}\right)\right|_{\pi}>0
\end{array}
$$

where $\varphi$ is an arbitrary constant phase, and $J$ and $\Omega_{3}$ are the covariantly constant Kähler two-form and holomorphic three-form, respectively, that always exist on a Calabi-Yau threefold (and, in particular, on the $T^{6} / \mathbb{Z}_{4}$ orbifold we consider here). They can be defined 
locally by

$$
\Omega_{3}=d z^{1} \wedge d z^{2} \wedge d z^{3}, \quad J=i \sum_{i, \bar{j}} g_{i \bar{j}} d z^{i} \wedge d \bar{z}^{\bar{j}} .
$$

If two Lagrangian three-cycles are calibrated by different values of the phase $\varphi$, the corresponding D6-branes preserve different supersymmetries. Since the three-cycle wrapped by the O6-plane is automatically $s \operatorname{Lag}$, the phase $\varphi$ is fixed to $\varphi=\varphi_{\mathrm{O} 6}$, and we need to search for the Lagrangian three-cycles which are calibrated by $\operatorname{Re}\left(e^{i \varphi} \mathrm{O}_{0} \Omega_{3}\right)$.

In terms of the $\vec{\theta}$, the angle $\varphi_{\mathrm{O} 6}$ is given by

$$
\varphi_{\mathrm{O} 6}=-\frac{1}{2} \sum_{i} \theta_{i}(+\pi)
$$

The possible $+\pi$ in the previous equation arises from the freedom we have to choose either $\int\left(e^{i \varphi} \Omega_{3}\right)>0$ or $\int\left(e^{i \varphi+i \pi} \Omega_{3}\right)>0$ as the calibration condition, or, alternatively, the global sign of the three-cycle wrapped by the O6-plane.

$\boldsymbol{B}_{\mathbf{2}} \times\left(\boldsymbol{A}_{\mathbf{1}}\right)^{\mathbf{2}} \times \boldsymbol{B}_{\mathbf{2}}$. Due to the factorisable structure of the $B_{2} \times\left(A_{1}\right)^{2} \times B_{2}$-lattice, any three-cycle with the wrapping numbers $\left(n^{i}, m^{i}\right)_{i=1,2,3}$ is automatically Lagrangian. The condition that a D6-brane wrapping such a three-cycle preserves the same supersymmetry as the O6-plane (i.e. is $s L a g$ with identical calibration) is

$$
\sum_{i} \phi_{i}=0 \bmod 2 \pi
$$

where $\phi_{i}$ is an angle between the three-cycle and the O6-plane on the two-torus $T_{(i)}^{2}$. Fractional three-cycles with the same calibration arise when (fractions of) exceptional three-cycles are added through which a given bulk cycle passes with the one restriction on the relative prefactor discussed below equation (2.10); for more details see [34].

$\boldsymbol{A}_{\mathbf{3}} \times \boldsymbol{A}_{\mathbf{3}}$. With respect to the complex coordinates (3.4), the $Q$-invariant metric $g$ of equation (2.13) is given by

$$
g_{i \bar{j}}=\left(\begin{array}{ccc}
2 R_{1}^{2}(1+a) & 0 & R_{1} R_{2}(e-c-i(c+2 d+e)) \\
0 & -8 u_{2} a R_{1}^{2} & 0 \\
R_{1} R_{2}(e-c+i(c+2 d+e)) & 0 & 2 R_{2}^{2}(1+b)
\end{array}\right) .
$$

The Kähler-form $J$ on the $A_{3} \times A_{3}$-lattice background is given by

$$
J:=2 R_{1}^{2}(1+a) \omega_{1}-2 u_{2} a R_{1}^{2} \omega_{2}+2 R_{2}^{2}(1+b) \omega_{3}+R_{1} R_{2}\left((c+2 d+e) \omega_{4}+(c-e) \omega_{5}\right)
$$

with real $\mathbb{Z}_{4}$-invariant two-forms $\omega_{i}$ :

$$
\begin{aligned}
& \omega_{1}:=d x^{12}+d x^{23}, \\
& \omega_{2}:=d x^{14}-d x^{15}+d x^{16}-d x^{24}+d x^{25}-d x^{26}+d x^{34}-d x^{35}+d x^{36}, \\
& \omega_{3}:=d x^{45}+d x^{56} \\
& \omega_{4}:=d x^{14}-d x^{16}+d x^{25}-d x^{34}+d x^{36}, \\
& \omega_{5}:=-d x^{15}+d x^{24}-d x^{26}+d x^{35},
\end{aligned}
$$

where $d x^{i j}:=d x^{i} \wedge d x^{j}$. 


\begin{tabular}{|c|c|c|c|c|}
\hline \multicolumn{5}{|c|}{$A_{3} \times A_{3}$-orientifolds } \\
\hline Lattice & Involution & Invariant part of $J$ & Moduli fixing & $\varphi_{\mathrm{O} 6_{i}}$ \\
\hline AAA & $\mathcal{R}_{1}$ & $\omega_{4}$ & $d=0$ & 0 \\
\hline BAA & $\mathcal{R}_{2}$ & $\omega_{4}+\omega_{5}$ & $e=d$ & $-\frac{\pi}{4}$ \\
\hline AAB & $\mathcal{R}_{3}$ & $\omega_{4}-\omega_{5}$ & $e=-d$ & $-\frac{\pi}{4}$ \\
\hline BAB & $\mathcal{R}_{4}$ & $\omega_{4}$ & $d=0$ & $\frac{\pi}{2}$ \\
\hline
\end{tabular}

Table 12. Additional moduli fixing and calibration arising from the condition $\mathcal{R} J=-J$ for the different choices of $A_{3} \times A_{3}$ lattice orientations.

The Kähler two-form is negative under the orientifold projection, i.e. $\mathcal{R} J=-J$. This means that the $\mathcal{R}$-even part of $J$ has to vanish, which fixes part of the moduli, in addition to the fixing $u_{1}=0$ (i.e. $c+e=0$ ) mentioned in the previous subsection. The additional moduli fixing for the different orientifold projections is given in table 12 .

While, in the factorisable case, i.e. $T^{6}=\left(T^{2}\right)^{3}$, all factorised three-cycles are Lagrangian, this does not hold true anymore on the orbifolds with some non-factorisable lattice. However, if we fix the moduli $c=d=e=0$, i.e. both $A_{3}$-tori are orthogonal to each other, and use the relation $d x^{i}\left(\pi_{j}\right)=\delta_{j}^{i}$, we can verify that any toroidal $\mathbb{Z}_{2}$-invariant three-cycle (2.22) is automatically Lagrangian.

The next step is to specify the $s L a g$ condition on our orientifolds $T^{6} /\left(\mathbb{Z}_{4} \times \Omega \mathcal{R}_{i}\right)$ in order to find the supersymmetric three-cycles. The holomorphic three-form $\Omega_{3}=\operatorname{Re} \Omega_{3}+i \operatorname{Im} \Omega_{3}$ has the decomposition

$$
\begin{aligned}
& \operatorname{Re}\left(\Omega_{3}\right)=\frac{1}{8 \sqrt{u_{2}}}\left(\rho_{1}-u_{2} \rho_{4}\right), \\
& \operatorname{Im}\left(\Omega_{3}\right)=\frac{1}{8 \sqrt{u_{2}}}\left(\rho_{2}+u_{2} \rho_{3}\right),
\end{aligned}
$$

with real $\mathbb{Z}_{4}$-invariant three-forms

$$
\begin{aligned}
& \rho_{1}:=-d x^{124}+d x^{125}+d x^{126}+2 d x^{134}-2 d x^{136}-d x^{234}-d x^{235}+d x^{236}, \\
& \rho_{2}:=-d x^{124}-d x^{125}+d x^{126}+2 d x^{135}+d x^{234}-d x^{235}-d x^{236}, \\
& \rho_{3}:=d x^{145}-2 d x^{146}+d x^{156}-d x^{245}+d x^{256}-d x^{345}+2 d x^{346}-d x^{356}, \\
& \rho_{4}:=d x^{145}-d x^{156}+d x^{245}-2 d x^{246}+d x^{256}-d x^{345}+d x^{356} .
\end{aligned}
$$

Because this case is not so interesting from the phenomenological point of view, we do not go into detail here and only give the supersymmetry parameters $\varphi_{\mathrm{O} 6_{i}}$ in table 12 for each involution.

$\boldsymbol{A}_{\mathbf{3}} \times \boldsymbol{A}_{\mathbf{1}} \times \boldsymbol{B}_{\mathbf{2}} . \quad$ With respect to the complex coordinates(3.13), the $A_{3} \times A_{1} \times B_{2}$-orbifold has the hermitian metric

$$
g_{i \bar{j}}=\left(\begin{array}{ccc}
2 R_{3}^{2}(1+a) & 0 & \frac{R_{2} R_{3}}{\sqrt{2}}(b+i(b+2 c)) \\
0 & -8 u_{2} a R_{3}^{2} & 0 \\
\frac{R_{2} R_{3}}{\sqrt{2}}(b-i(b+2 c)) & 0 & 2 R_{2}^{2}
\end{array}\right),
$$




\begin{tabular}{|c|c|c|c|c|}
\hline \multicolumn{5}{|c|}{$A_{3} \times A_{1} \times B_{2}$-orientifolds } \\
\hline Lattice & Involution & Invariant part of $J$ & Moduli fixing & $\varphi_{\mathrm{O} 6_{i}}$ \\
\hline AAB & $\mathcal{R}_{1}$ & $\omega_{4}$ & $b=-2 c$ & 0 \\
\hline AAA & $\mathcal{R}_{2}$ & $\omega_{4}+\omega_{5}$ & $b=-c$ & $\frac{5 \pi}{4}$ \\
\hline ABA & $\mathcal{R}_{3}$ & $\omega_{4}$ & $b=-2 c$ & $\frac{3 \pi}{2}$ \\
\hline ABB & $\mathcal{R}_{4}$ & $\omega_{4}-\omega_{5}$ & $c=0$ & $\frac{\pi}{4}$ \\
\hline
\end{tabular}

Table 13. Moduli fixing due to $\mathcal{R} J=-J$ and calibration for different choices of the antiholomorphic involution on the $A_{3} \times A_{1} \times B_{2}$ lattice.

which gives rise to the Kähler-form

$$
J=2 R_{3}^{2}(1+a) \omega_{1}+2 R_{2}^{2} \omega_{2}-4 R_{3}^{2} a u_{2} \omega_{3}-R_{3} R_{2}(b+2 c) \omega_{4}-R_{3} R_{2} b \omega_{5}
$$

with the following $\mathbb{Z}_{4}$-invariant two-forms:

$$
\begin{aligned}
& \omega_{1}:=d x^{12}+d x^{23} \\
& \omega_{2}:=d x^{56} \\
& \omega_{3}:=d x^{14}-d x^{24}+d x^{34} \\
& \omega_{4}:=2 d x^{15}-d x^{16}+d x^{26}-2 d x^{35}+d x^{36}, \\
& \omega_{5}:=-d x^{16}+2 d x^{25}-d x^{26}+d x^{36} .
\end{aligned}
$$

The anti-symmetry of the orientifold projection on $J$ leads here again to the fixing of some moduli, as shown in the table 13 .

As in the $A_{3} \times A_{3}$-case, we verify that any toroidal $\mathbb{Z}_{2}$-invariant three-cycle (2.34) is automatically Lagrangian for the choice of fixing the moduli to $b=c=0$.

Using the complex coordinates (3.13), we can write $\Omega_{3}$ in real coordinates

$$
\begin{aligned}
& \operatorname{Re}\left(\Omega_{3}\right)=\frac{1}{4 \sqrt{u_{2}}}\left(\rho_{1}+u_{1} \rho_{3}-u_{2} \rho_{4}\right), \\
& \operatorname{Im}\left(\Omega_{3}\right)=\frac{1}{4 \sqrt{u_{2}}}\left(\rho_{2}+u_{2} \rho_{3}+u_{1} \rho_{4}\right),
\end{aligned}
$$

where the $\mathbb{Z}_{4}$-invariant three-forms are:

$$
\begin{aligned}
& \rho_{1}:=-d x^{125}+d x^{126}-d x^{136}+2 d x^{135}-d x^{235}, \\
& \rho_{2}:=-d x^{125}+d x^{136}+d x^{235}-d x^{236}, \\
& \rho_{3}:=2 d x^{145}-d x^{146}-d x^{246}-2 d x^{345}+d x^{346}, \\
& \rho_{4}:=d x^{146}+2 d x^{245}-d x^{246}-d x^{346} .
\end{aligned}
$$

The next step is to find the $s L a g$ s on the orientifolds $T^{6} /\left(\mathbb{Z}_{4} \times \Omega \mathcal{R}_{i}\right)$ with $A_{3} \times A_{1} \times B_{2}$ lattice. The sLag condition for any three-cycle (2.26) inherited from the torus can be 
expressed as follows,

$$
\begin{aligned}
& \left.\operatorname{Im}\left(\Omega_{3}\right)\right|_{\pi^{\text {torus }}}=\frac{1}{4 \sqrt{u_{2}}}\left([Q-P]+u_{1}[\bar{P}-\bar{Q}]-u_{2}[\bar{P}+\bar{Q}]\right), \\
& \left.\operatorname{Re}\left(\Omega_{3}\right)\right|_{\pi^{\text {torus }}}=\frac{1}{4 \sqrt{u_{2}}}\left([P+Q]-u_{1}[\bar{P}+\bar{Q}]-u_{2}[\bar{P}-\bar{Q}]\right),
\end{aligned}
$$

where $P, Q, \bar{P}, \bar{Q}$ are the bulk wrapping numbers defined in (2.28). Using this decomposition of a (fraction of a) bulk three-cycle into real and imaginary part in dependence of the complex structure $u$ and the bulk wrapping numbers for the corresponding O6-planes we can calculate the supersymmetry parameters $\varphi_{\mathrm{O} 6_{i}}$ per lattice orientation displayed in table 13 .

\subsection{Pairwise relations between choices of orientifold axes}

We know that in the factorisable case a priori six choices of the anti-holomorphic involution are possible, but that there are pairwise relations between them so that only four are physically inequivalent. This means that different but equivalent orientifold projections give rise to the same global semi-realistic particle models as discussed in section 3.1.1.

The same observation can be made in the non-factorisable cases. In order to find these relations between the lattices, we compute all supersymmetric three-cycles which do not overshoot the bulk RR tadpole cancellation condition in (3.1). Together with the supersymmetry conditions (3.24) this gives rise to the restriction on the toroidal and corresponding bulk wrapping numbers, e.g. for the $\mathbf{A} \mathbf{a} \mathbf{A B}$-lattice of the $A_{3} \times A_{1} \times B_{2}$ lattice, they are bounded by

$$
\begin{aligned}
A_{1} n^{3}-A_{2} n^{3}+2 A_{2} m^{3} \leqslant 8, \\
2 B_{3} n^{3}-2 B_{2} m^{3} \leqslant 16,
\end{aligned} \Longleftrightarrow \quad \begin{aligned}
& P+Q \leqslant 8 \\
& \bar{Q}-\bar{P} \leqslant 16 .
\end{aligned}
$$

The restrictions on the other lattice orientations take a similar form. The required O6plane bulk wrapping numbers entering (3.1) are given in (3.12) for the $A_{3} \times A_{3}$ lattice, and in (3.23) for the $A_{3} \times A_{1} \times B_{2}$ lattice.

$\boldsymbol{A}_{\mathbf{3}} \times \boldsymbol{A}_{\mathbf{3}} . \quad$ For this orbifold we found a priori four possible involutions. However, due to the relation $\mathcal{R}_{4} Q^{3}=-\mathcal{R}_{1}$ between the involutions $\mathcal{R}_{1}$ and $\mathcal{R}_{4}$ the $\mathbf{A A A}$ - and $\mathbf{B} \mathbf{A B}$-lattices give physically identical models on the $A_{3} \times A_{3}$-orientifold. But the relation between the corresponding complex structure values cannot be verified more precisely at this point. There are two possibilities

$$
\begin{aligned}
\text { AAA dual to } \mathbf{B A B} \text { and } u_{2} & =u_{2}^{\prime}, \\
\text { or } & \text { AAA dual to } \mathbf{B A B} \text { and } u_{2}=\frac{1}{u_{2}^{\prime}} \text {. }
\end{aligned}
$$

Moreover, the orientifold projections $\mathcal{R}_{2}$ and $\mathcal{R}_{3}$ - corresponding to the $\mathbf{B A A}$ and $\mathbf{A A B}$ lattice orientation, respectively, according to table 12 - act on the three-cycles in the same way, and therefore the supersymmetric three-cycles on the corresponding orientifolds are the same. At first sight one might be tempted to identify these orientifolds, but the 


\begin{tabular}{|c|c|c|c|}
\hline \multicolumn{4}{|c|}{$A_{3} \times A_{1} \times B_{2}$-orientifolds } \\
\hline Lattice & Orien. proj. & \# of frac. cycles & \# of $u_{2}$ \\
\hline $\mathbf{A}_{\mathbf{a}} \mathbf{A A}$ & $\mathcal{R}_{2}\left(u_{1}=0\right)$ & 2126 & 96 \\
\hline $\mathbf{A}_{\mathbf{a}} \mathbf{A B}$ & $\mathcal{R}_{1}\left(u_{1}=0\right)$ & 2126 & 96 \\
\hline $\mathbf{A}_{\mathbf{a}} \mathbf{B A}$ & $\mathcal{R}_{3}\left(u_{1}=0\right)$ & 5134 & 210 \\
\hline $\mathbf{A}_{\mathbf{a}} \mathbf{B B}$ & $\mathcal{R}_{4}\left(u_{1}=0\right)$ & 5134 & 210 \\
\hline \hline $\mathbf{A}_{\mathbf{b}} \mathbf{A A}$ & $\mathcal{R}_{2}\left(u_{1}=\frac{1}{2}\right)$ & 2410 & 118 \\
\hline $\mathbf{A}_{\mathbf{b}} \mathbf{A B}$ & $\mathcal{R}_{1}\left(u_{1}=\frac{1}{2}\right)$ & 3646 & 140 \\
\hline $\mathbf{A}_{\mathbf{b}} \mathbf{B A}$ & $\mathcal{R}_{3}\left(u_{1}=\frac{1}{2}\right)$ & 3646 & 140 \\
\hline $\mathbf{A}_{\mathbf{b}} \mathbf{B B}$ & $\mathcal{R}_{4}\left(u_{1}=\frac{1}{2}\right)$ & 2410 & 118 \\
\hline
\end{tabular}

Table 14. The number of supersymmetric fractional cycles bounded by the bulk RR tadpole cancellation condition and the number of possible complex structure values $u_{2}$ for different choices of orientifold axes.

distinction of the length of the O6-planes, $\pi_{\mathrm{O}_{2}}=2 \pi_{\mathrm{O} 6_{3}}$, gives in principle rise to different allowed ranks and lengths of bulk cycles in the RR tadpole cancellation conditions and consequently to more possible models for the BAA-orientifold.

In conclusion, by investigating the structure of bulk three-cycles and their RR tadpole cancellation conditions, we arrive at three physically inequivalent $A_{3} \times A_{3}$-lattice orientations AAA, BAA and AAB.

$\boldsymbol{A}_{\mathbf{3}} \times \boldsymbol{A}_{\mathbf{1}} \times \boldsymbol{B}_{\mathbf{2}}$. In this case there are a priori eight possible lattice orientations. The number of the corresponding fractional cycles not overshooting the bulk RR tadpole cancellation conditions and the number of possible complex structure values $u_{2}$ are presented in the table 14 .

Furthermore, we can easily verify the following pairwise relations between the different lattice orientations:

$$
\begin{aligned}
& \mathbf{A}_{\mathbf{a}} \mathbf{A} \mathbf{A} \text { dual to } \mathbf{A}_{\mathbf{a}} \mathbf{A B} \text { and } u_{2}=\frac{1}{2 u_{2}^{\prime}}, \\
& \mathbf{A}_{\mathbf{a}} \mathbf{B A} \text { dual to } \mathbf{A}_{\mathbf{a}} \mathbf{B B} \text { and } u_{2}=\frac{1}{2 u_{2}^{\prime}}, \\
& \mathbf{A}_{\mathbf{b}} \mathbf{A A} \text { dual to } \mathbf{A}_{\mathbf{b}} \mathbf{B B} \text { and } u_{2}=\frac{1}{4 u_{2}^{\prime}}, \\
& \mathbf{A}_{\mathbf{b}} \mathbf{B} \mathbf{A} \text { dual to } \mathbf{A}_{\mathbf{b}} \mathbf{A B} \text { and } u_{2}=\frac{1}{4 u_{2}^{\prime}} .
\end{aligned}
$$

Altogether, we have thus four physically inequivalent lattices (two with $u_{1}=0$ and two with $u_{1}=\frac{1}{2}$ ) and can restrict our further considerations to the lattice orientations $\mathrm{AAB}$ and $\mathrm{ABB}$. 


\subsection{Cross-check: D6-branes on top of O6-planes}

Non-factorisable $T^{6} / \mathbb{Z}_{4}$ orbifolds have been briefly studied in the past. For instance, in [63] only D6-branes on top of the orientifold planes were considered, and only for a particular choice of the moduli in (2.13) and (2.25), i.e., the radii of all tori being equal and all the tori being orthogonal to each other. Our goal in this section is twofold. At first, we will reproduce and extend the CFT methods and results of [63] to arbitrary values of the moduli, in particular providing the Kaluza-Klein and winding modes for generic D6-brane configuration. Secondly, we will compare this result with the geometric method described earlier on, i.e. by requiring that the $s L a g$ three-cycles wrapped by generic D6-branes satisfy the RR tapdole cancellation condition (3.1) - in the case at hand with only two stacks of D6-branes.

Let us start by reviewing the method used in [63]. We denote a basis of the torus lattice by $\left\{e_{i}\right\}$. Let $\left\{e_{i}^{*}\right\}$ be a basis of the dual lattice such that $e_{i} \cdot e_{j}^{*}=\delta_{i j}$. If the lattice vectors $e_{i}$ form the $Q$-invariant metric $g_{i j}$, the dual vectors $\left\{e_{i}^{*}\right\}$ form the metric $g_{i j}^{*}=e_{i}^{*} \cdot e_{j}^{*}=g_{i j}^{-1}$. Note that the dual vectors transform under $Q^{t}$ and $\mathcal{R}_{m(m=1,2,3,4)}^{t}$.

In general, insertions of $\Omega \mathcal{R}_{m} Q^{2 k}$ and $\Omega \mathcal{R}_{m} Q^{2 k+1}$ in the Klein bottle trace (and strings starting on the $6_{2 k}$ and $6_{2 k+1}$ branes in the annulus and Möbius strip) give different lattice contributions, so we need to compute both cases separately. In the rest of this section we will only consider the first case, but the second one is obtained analogously, by replacing $\mathcal{R}_{m}$ by $\mathcal{R}_{m} Q$ throughout the elaboration.

Let $\mathbf{v}_{i}(i=1,2,3)$ be the lattice vectors that span the (fraction of the) bulk three-cycle wrapped by the O6-plane. They satisfy $\mathcal{R}_{m} \mathbf{v}_{i}=\mathbf{v}_{i}$. The winding modes are described by vectors $\mathbf{w}_{i}, i=1,2,3$, satisfying $\mathcal{R}_{m} \mathbf{w}_{i}=-\mathbf{w}_{i}$. The momentum modes $\mathbf{p}_{i}$ appearing in the Klein bottle amplitude in sectors where there are fixed tori correspond to vectors in the dual lattice invariant under $\mathcal{R}_{m}$. Using all these vectors we define the matrices

$$
\begin{aligned}
\left(M_{K B}\right)_{i j}:=\mathbf{p}_{i} \cdot \mathbf{p}_{j} \\
\left(M_{A}\right)_{i j}=\left(M_{M S}\right)_{i j}:=\mathbf{v}_{i} \cdot \mathbf{v}_{j} \\
\left(W_{K B}\right)_{i j}=\left(W_{M S}\right)_{i j}:=\mathbf{w}_{i} \cdot \mathbf{w}_{j}
\end{aligned}
$$

The lattice mode contributions (in the corresponding sector) for the Klein bottle, annulus, and Möbius strip are

$$
\begin{aligned}
K B & =\frac{4^{n}}{\left(\operatorname{det} M_{K B} \operatorname{det} W_{K B}\right)^{1 / 2}}, \\
A & =\frac{\operatorname{det} M_{A}}{(\operatorname{det} g)^{1 / 2}}, \\
M S & =\frac{4^{n}\left(\operatorname{det} M_{M S}\right)^{1 / 2}}{\left(\operatorname{det} W_{M S}\right)^{1 / 2}},
\end{aligned}
$$

where $n=\operatorname{dim}(M)=\operatorname{dim}(W),(n=3$ in the untwisted sector, which is the one we are interested in). 
The tadpole cancellation condition is then given by

$$
K B+\frac{M^{2} \cdot A}{16}-\frac{M \cdot M S}{16}=0
$$

where $M$ is the number of identical branes (in the $\mathcal{R}_{m} Q$ case we will denote it by $N$ ).

$\boldsymbol{A}_{\mathbf{3}} \times \boldsymbol{A}_{\mathbf{3}}$. Let us start with the $A_{3} \times A_{3}$ lattice. For concreteness, we will focus on the orientation AAB. We will show that the number of supersymmetric D6-branes needed to cancel the (bulk) RR tadpole depends on the angle-moduli between the two $A_{3}$-tori.

Recall that for the orientifold AAB we have the involution $\mathcal{R}_{3}$ (3.7), which fixes the moduli to $c+e=0$ (as consequence of $u_{1}=0$ ) and $e+d=0$ (see table 12). Using these constraints and the $Q$-invariant metric $g$, we obtain the following momentum modes $\mathbf{p}_{i}$ :

$$
\begin{aligned}
& \mathbf{p}_{1}=2 e_{1}^{*}-e_{2}^{*}, \\
& \mathbf{p}_{2}=e_{1}^{*}-e_{3}^{*}, \\
& \mathbf{p}_{3}=e_{4}^{*}+e_{5}^{*}-e_{6}^{*} .
\end{aligned}
$$

The winding modes are:

$$
\begin{aligned}
& \mathbf{w}_{1}=e_{1}+2 e_{2}+e_{3}, \\
& \mathbf{w}_{2}=e_{4}-e_{5}, \\
& \mathbf{w}_{3}=e_{4}+e_{6} .
\end{aligned}
$$

The $\mathbf{v}_{i}$ vectors spanning the O6-planes are:

$$
\begin{aligned}
& \mathbf{v}_{1}=e_{1}, \\
& \mathbf{v}_{2}=e_{3}, \\
& \mathbf{v}_{3}=e_{4}+e_{5} .
\end{aligned}
$$

The determinants of the corresponding matrices $M_{K B}, M_{A}$ and $W_{K B}$ are

$$
\begin{aligned}
\operatorname{det} M_{K B} & =-\frac{2}{a\left(1+a+b+a b-2 e^{2}\right) R_{1}^{4} R_{2}^{2}}, \\
\operatorname{det} M_{A} & =-8 a\left(1+a+b+a b-2 e^{2}\right) R_{1}^{4} R_{2}^{2}, \\
\operatorname{det} W_{K B} & =-32 b\left(1+a+b+a b-2 e^{2}\right) R_{1}^{2} R_{2}^{4} .
\end{aligned}
$$

They give rise to the lattice mode contributions to the untwisted sector

$$
K B=8 \sqrt{\frac{a}{b}} \frac{R_{1}}{R_{2}}, \quad A=2 \sqrt{\frac{a}{b}} \frac{R_{1}}{R_{2}}, \quad M S=32 \sqrt{\frac{a}{b}} \frac{R_{1}}{R_{2}} .
$$

The untwisted RR tadpole cancellation condition is

$$
K B+\frac{M^{2} \cdot A}{16}-\frac{M \cdot M S}{16}=0 \Rightarrow(M-8)^{2}=0 .
$$

The $\mathcal{R}_{3} Q$-case leads to the analogous result $(N-8)^{2}=0$. Due to the action of the $\mathbb{Z}_{2}$ symmetry on the corresponding Chan-Paton factors, the gauge group will have rank $\frac{M}{2} \times \frac{N}{2}$, 


\begin{tabular}{|c|c|c|c|c|}
\hline \multicolumn{5}{|c|}{$A_{3} \times A_{3}$ orientifolds with $D$-branes on top of the O-plane } \\
\hline \multicolumn{2}{|c|}{$A_{3} \times A_{3}$} & General & \multicolumn{2}{c|}{$e=0$} \\
\hline Lattice & Involution & $M=N$ & $M=N$ & Gauge group \\
\hline AAA & $\mathcal{R}_{1}$ & 8 & 8 & $\mathrm{USp} / \mathrm{SO}(8) \times \mathrm{USp} / \mathrm{SO}(8)$ \\
\hline BAA & $\mathcal{R}_{2}$ & 8 & 8 & $\mathrm{USp} / \mathrm{SO}(8) \times \mathrm{USp} / \mathrm{SO}(8)$ \\
\hline AAB & $\mathcal{R}_{3}$ & 8 & 8 & $\mathrm{USp} / \mathrm{SO}(8) \times \mathrm{USp} / \mathrm{SO}(8)$ \\
\hline BAB & $\mathcal{R}_{4}$ & 8 & 8 & $\mathrm{USp} / \mathrm{SO}(8) \times \mathrm{USp} / \mathrm{SO}(8)$ \\
\hline
\end{tabular}

Table 15. IIA Orientifolds of $\mathbb{Z}_{4}$-orbifolds with the lattice of the type $A_{3} \times A_{3}$ with D-branes on O-plane. For generic values of the metric moduli, the gauge group is $\mathrm{U}\left(\frac{M}{2}\right) \times \mathrm{U}\left(\frac{N}{2}\right)$.

and the gauge group for generic values of moduli is $\mathrm{U}(4) \times \mathrm{U}(4)$ (with a possible gauge symmetry enhancement $\mathrm{U}(4) \hookrightarrow \mathrm{USp} / \mathrm{SO}(8),{ }^{6}$ for special choices of geometric moduli in particular for the choice $c=d=e=0$ of the two $A_{3}$ lattices orthogonal to each other in $(2.13)$ - , where determining the appropriate type of symmetry enhancement requires the development of CFT techniques at one-loop so far only available for $\left(T^{2}\right)^{3}$ factorisable backgrounds, see e.g. [37, 73, 79, 80, 96]).

The complementary purely geometric considerations are as follows: the cycle wrapped by the O6-plane is $-2 \gamma_{1}-2 \bar{\gamma}_{2}$. For $e=0$, its contribution to the tadpole can be cancelled by a stack of $N_{1}=4$ branes wrapping the cycle $-\gamma_{1}$ and a second stack of $N_{2}=4$ branes wrapping $-\bar{\gamma}_{2}$, giving rise to a gauge group $\mathrm{USp} / \mathrm{SO}(8) \times \mathrm{USp} / \mathrm{SO}(8)$ in agreement with the CFT result.

The results for the remaining orientifold projections are summarised in the table 15.

$\boldsymbol{A}_{\mathbf{3}} \times \boldsymbol{A}_{\mathbf{1}} \times \boldsymbol{B}_{\mathbf{2}}$. Let us now consider the $A_{3} \times A_{1} \times B_{2}$ lattice. For simplicity we will present the analysis of the case where the $B_{2}$-torus is orthogonal to $A_{3} \times A_{1}$. This means that the moduli $b, c$ vanish. ${ }^{7}$ For the explicit computations, we will focus on the $\mathcal{R}_{1}$ involution (3.15).

$\mathbf{A}_{\mathbf{a}} \mathbf{A B}$. The condition $u_{1}=0$ restricts the moduli $d$ and $a$. From the definition of the complex structure modulus (3.14) we obtain that $d$ is fixed to 0 and $a$ has to be negative.

Since the dual basis vectors $e_{i}^{*}$ transform under the transposed orientifold action $\mathcal{R}_{1}^{t}$, the momentum modes $\mathbf{p}_{i}$ are the eigenvectors of $\mathcal{R}_{1}^{t}$ to the eigenvalue +1 :

$$
\begin{aligned}
& \mathbf{p}_{1}=e_{1}^{*}-e_{3}^{*}, \\
& \mathbf{p}_{2}=2 e_{1}^{*}-e_{2}^{*}+e_{3}^{*}, \\
& \mathbf{p}_{3}=e_{5}^{*}+e_{6}^{*}
\end{aligned}
$$

\footnotetext{
${ }^{6}$ This is a shorthand notation for either $\mathrm{USp}(8)$ or $\mathrm{SO}(8)$.

${ }^{7}$ For an arbitrary choice of these moduli (up to the moduli fixing in table 13), we obtain the same results.
} 
The winding modes are

$$
\begin{aligned}
& \mathbf{w}_{1}=e_{1}+2 e_{2}+e_{3}, \\
& \mathbf{w}_{2}=e_{4}, \\
& \mathbf{w}_{3}=e_{5}-e_{6},
\end{aligned}
$$

and the $\mathbf{v}_{i}$ vectors describing the bulk part of a fractional cycle parallel to some O6-plane are

$$
\begin{aligned}
& \mathbf{v}_{1}=e_{1}, \\
& \mathbf{v}_{2}=e_{3}, \\
& \mathbf{v}_{3}=e_{5}+e_{6} .
\end{aligned}
$$

The determinants of the different matrices are

$$
\operatorname{det} M_{K B}:=-\frac{2}{a(1+a) R_{3}^{4} R_{2}^{2}}, \quad \operatorname{det} M_{A}:=-8 a(1+a) R_{3}^{4} R_{2}^{2}, \quad \operatorname{det} W_{K B}:=8(1+a) R_{3}^{2} R_{2}^{2} R_{1}^{2} .
$$

The lattice mode contributions to the untwisted sector are

$$
K B=16 \sqrt{-a} \frac{R_{3}}{R_{1}}, \quad A=4 \sqrt{-a} \frac{R_{3}}{R_{1}}, \quad M S=64 \sqrt{-a} \frac{R_{3}}{R_{1}},
$$

and the corresponding untwisted $\mathrm{RR}$ tadpole cancellation condition reads

$$
K B+\frac{M^{2} \cdot A}{16}-\frac{M \cdot M S}{16}=0 \Rightarrow(M-8)^{2}=0 .
$$

Thus, we get $M=8$ which agrees with [63]. In a similar way one shows that the tadpole condition from the $\mathcal{R}_{1} Q$-part gives rise to $N=16$. The resulting gauge group is thus $\mathrm{U}(4) \times \mathrm{U}(8)$ or some rank preserving gauge enhancement to $\mathrm{SO}(2 M)$ or $\mathrm{USp}(2 M)$ (for one or both gauge factors).

The CFT calculation is complemented by purely geometric considerations as follows: the cycle wrapped by the O6-planes is $\gamma_{1}+\gamma_{2}-2\left(\bar{\gamma}_{1}-\bar{\gamma}_{2}\right)$. Its contribution to the tadpole can be cancelled by a stack of $N_{1}=4$ branes wrapping the fractional cycle $\frac{1}{2} \gamma_{1}+\frac{1}{2} \gamma_{2}$ and a stack of $N_{2}=8$ branes wrapping $-\frac{1}{2} \bar{\gamma}_{1}+\frac{1}{2} \bar{\gamma}_{2} \pm \frac{1}{2} \bar{\gamma}_{3} \pm \frac{1}{2} \bar{\gamma}_{4}$ (any choice of sign for the exceptional part is allowed). Since the first stack is invariant under the orientifold action, the actual gauge group is $\mathrm{SO} / \mathrm{USp}(8) \times \mathrm{U}(8)$. Our result — which agrees for both $(\mathrm{CFT}$ and cycle homology) methods - differs slightly from [63] in the fact that the first gauge factor experiences a gauge group enhancement, which they do not mention.

$\mathbf{A}_{\mathbf{b}} \mathbf{A B}$. In this case, from the condition $u_{1}=\frac{1}{2}$ we obtain

$$
d=-\frac{a R_{3}}{R_{1}}, \quad a\left(1+a \frac{R_{3}^{2}}{R_{1}^{2}}\right)<0 .
$$

The momentum modes $\mathbf{p}_{i}$ are

$$
\begin{aligned}
& \mathbf{p}_{1}=e_{1}^{*}-e_{3}^{*}, \\
& \mathbf{p}_{2}=2 e_{1}^{*}-e_{2}^{*}+e_{4}^{*}, \\
& \mathbf{p}_{3}=e_{6}^{*}
\end{aligned}
$$




\begin{tabular}{|c|c|c|c|c|c|c|c|}
\hline \multicolumn{7}{|c|}{$A_{3} \times A_{1} \times B_{2}$ orientifolds with D-branes on top of the O-planes } \\
\hline \multicolumn{2}{|c|}{$A_{3} \times A_{1} \times B_{2}$} & \multicolumn{7}{|c|}{$u_{1}=0$} & \multicolumn{5}{c|}{$u_{1}=\frac{1}{2}$} \\
\hline Lattice & Involution & $M$ & $N$ & Gauge group & $M$ & $N$ & Gauge group \\
\hline AAA & $\mathcal{R}_{2}$ & 16 & 8 & $\mathrm{U}(8) \times \mathrm{USp} / \mathrm{SO}(8)$ & 16 & 4 & $\mathrm{U}(8) \times \mathrm{USp} / \mathrm{SO}(4)$ \\
\hline AAB & $\mathcal{R}_{1}$ & 8 & 16 & $\mathrm{USp} / \mathrm{SO}(8) \times \mathrm{U}(8)$ & 8 & 8 & $\mathrm{USp} / \mathrm{SO}(8) \times \mathrm{U}(4)$ \\
\hline ABA & $\mathcal{R}_{3}$ & 16 & 8 & $\mathrm{U}(8) \times \mathrm{USp} / \mathrm{SO}(8)$ & 8 & 8 & $\mathrm{U}(8) \times \mathrm{USp} / \mathrm{SO}(8)$ \\
\hline ABB & $\mathcal{R}_{4}$ & 8 & 16 & $\mathrm{USp} / \mathrm{SO}(8) \times \mathrm{U}(8)$ & 4 & 16 & $\mathrm{USp} / \mathrm{SO}(4) \times \mathrm{U}(8)$ \\
\hline
\end{tabular}

Table 16. IIA Orientifolds of $\mathbb{Z}_{4}$-orbifolds with the lattice of the type $A_{3} \times A_{1} \times B_{2}$ with D-branes on top of the O-planes canceling the RR tadpoles.

The winding modes $\mathbf{w}_{i}$ and lattice vectors $\mathbf{v}_{i}$ are

$$
\begin{aligned}
& \mathbf{w}_{1}=e_{1}+2 e_{2}+e_{3}, \\
& \mathbf{w}_{2}=e_{2}+e_{4}, \\
& \mathbf{w}_{3}=e_{5}, \\
& \mathbf{v}_{1}=e_{1}, \\
& \mathbf{v}_{2}=e_{3} \\
& \mathbf{v}_{3}=e_{5}+2 e_{6} .
\end{aligned}
$$

The determinants of the different matrices are thus given by

$$
\operatorname{det} M_{K B}=\frac{-2}{\left(a+a^{2}\right) R_{2}^{2} R_{3}^{4}}, \quad \operatorname{det} M_{A}=-8(1+a) a R_{2}^{2} R_{3}^{4}, \quad \operatorname{det} W_{K B}=8(1+a)\left(R_{1}^{2}+a R_{3}^{2}\right) R_{2}^{2} R_{3}^{2},
$$

and the resulting untwisted $\mathrm{RR}$ tadpole cancellation condition reads

$$
K B+\frac{M^{2} \cdot A}{16}-\frac{M \cdot M S}{16}=0 \Rightarrow(M-8)^{2}=0
$$

i.e. we obtain $M=8$. In a similar manner, the $\mathcal{R}_{1} Q$-insertion gives rise to $N=8$. Therefore, the gauge group is $\mathrm{U}(4) \times \mathrm{U}(4)$ or some rank preserving gauge group enhancement thereof.

The complementary considerations in terms of cycle homologies are as follows: the cycle wrapped by the O6-planes is $2 \gamma_{2}-2\left(\bar{\gamma}_{1}-\bar{\gamma}_{2}\right)$. Its contribution to the tadpole can be cancelled, for instance, by a stack of $N_{1}=4$ branes wrapping the fractional cycle $\frac{1}{2} \gamma_{1}+\frac{1}{2} \gamma_{2}$ and a stack of $N_{2}=4$ branes wrapping $-\frac{1}{2} \gamma_{1}+\frac{1}{2} \gamma_{2}-\bar{\gamma}_{1}+\bar{\gamma}_{2} \pm\left(\frac{1}{2} \gamma_{3}-\bar{\gamma}_{3}\right) \pm\left(\frac{1}{2} \gamma_{4}-\bar{\gamma}_{4}\right)$ (any choice of sign for the exceptional part is allowed). Naively, this gives rise to the gauge group $\mathrm{U}(4) \times \mathrm{U}(4)$, but the first stack maps to itself under the orientifold action, which enhances the symmetry to $\mathrm{USp} / \mathrm{SO}(8) \times \mathrm{U}(4)$.

The results for the remaining orientifold projections can be derived analogously and are summarised in the table 16 .

The column $u_{1}=0$ agrees mostly with the result [63], while the results for $u_{1}=\frac{1}{2}$ are presented here for the first time. We can also see the relation between the lattices (3.41) if we interchange $M$ and $N$. 


\section{Factorisation of non-factorisable orbifolds}

In this section we will show that both non-factorisable orbifolds can be written in a factorised form. This identification between the lattices was already detected in [97] by consideration of factorisable orbifolds. Here we want to explain which conclusions this identification means from the point of view of non-factorisable orbifolds. We make the restriction that in the first non-factorisable orbifold background, both $A_{3}$ lattices are orthogonal to each other, and in the second non-factorisable orbifold background, the $A_{3} \times A_{1}$ lattice is orthogonal to the $B_{2}$ lattice. This fixes the moduli to $c=d=e=0$ and $b=c=0$, respectively. On both orbifolds we can find a real basis such that the non-factorisable structure of the lattice decomposes into three two-tori, but an additional $\mathbb{Z}_{2}$ shift symmetry appears. Any three-cycle, written now as a product of three one-cycles on each two-torus, is automatically $\mathbb{Z}_{2}$-invariant. Moreover, only $\mathbb{Z}_{2}$-invariant three-cycles on the non-factorisable lattice can be expressed as three-cycles with respect to the new basis. Therefore, the number of wrapping numbers is reduced from twelve $\left(A_{3} \times A_{3}\right)$ or ten $\left(A_{3} \times A_{1} \times B_{2}\right)$ to six in agreement with the naive expectation from the known factorisable orbifold backgrounds $\left(T^{2}\right)^{3} / \mathbb{Z}_{N}$ or $\left(T^{2}\right)^{3} /\left(\mathbb{Z}_{N} \times \mathbb{Z}_{M}\right){ }^{8}$

Because any three-cycle, written in the new basis, is $\mathbb{Z}_{2}$-invariant, it follows that it is automatically Lagrangian. Furthermore, it can be verified that the $s$ Lag condition can be expressed in the same form as for the usual factorisable orbifolds: the sum of the angles between the one-cycles wrapped by supersymmetric D6-branes on each two-torus and the $\mathcal{R}_{i}$-invariant O6-plane has to vanish $(\bmod 2 \pi)$.

$\boldsymbol{A}_{\mathbf{3}} \times \boldsymbol{A}_{\mathbf{3}} . \quad$ In this case, we introduce new coordinates along the directions

$$
\begin{array}{lll}
v_{1}:=\pi_{1}+\pi_{2}, & v_{2}:=\pi_{2}+\pi_{3}, & v_{3}:=\pi_{1}+\pi_{3}, \\
v_{4}:=\pi_{4}+\pi_{6}, & v_{5}:=\pi_{4}+\pi_{5}, & v_{6}:=\pi_{5}+\pi_{6} .
\end{array}
$$

With respect to this basis, the metric $g$ and the Coxeter element $Q$ take a factorised form,

$$
\begin{aligned}
& g=\operatorname{diag}\left(2(1+a) R_{1}^{2}, 2(1+a) R_{1}^{2},-4 a R_{1}^{2},-4 b R_{2}^{2}, 2(1+b) R_{2}^{2}, 2(1+b) R_{2}^{2}\right), \\
& Q=\operatorname{diag}\left(\begin{array}{cc}
0 & -1 \\
1 & 0
\end{array}\right) \oplus\left(\begin{array}{cc}
-1 & 0 \\
0 & -1
\end{array}\right) \oplus\left(\begin{array}{cc}
0 & -1 \\
1 & 0
\end{array}\right)
\end{aligned}
$$

and also the orientifold projections $\mathcal{R}_{i}$ become factorised, e.g.:

$$
\mathcal{R}_{4}=\operatorname{diag}\left(\begin{array}{cc}
1 & 0 \\
0 & -1
\end{array}\right) \oplus\left(\begin{array}{cc}
1 & 0 \\
0 & -1
\end{array}\right) \oplus\left(\begin{array}{cc}
1 & 0 \\
0 & -1
\end{array}\right) .
$$

\footnotetext{
${ }^{8}$ Note, however, that due to the additional symmetry some of the new wrapping numbers can now also be half-integer.
} 

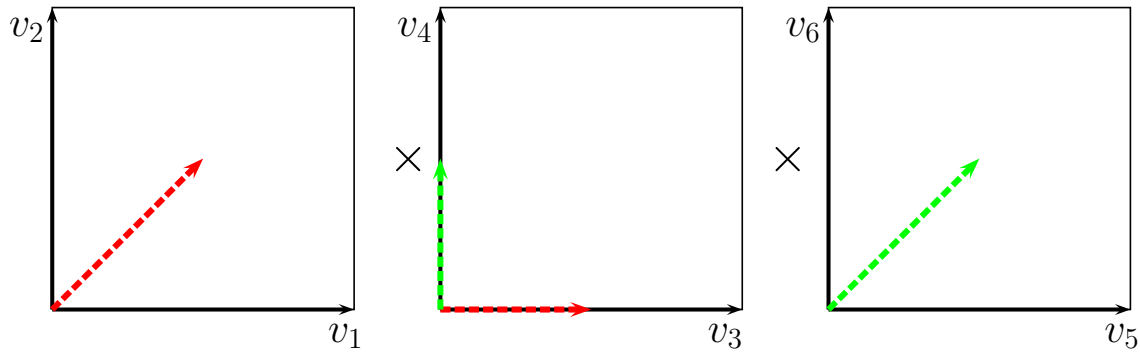

Figure 9. Shift identifications of points on the factorised form of the $A_{3} \times A_{3}$-lattice by $\frac{v_{1}+v_{2}+v_{3}}{2}$ (red) and $\frac{v_{4}+v_{5}+v_{6}}{2}$ (green).

But as already mentioned, the basis change gives rise to additional symmetries, which identify points of the factorised torus by the following shifts

$$
\begin{aligned}
& p \simeq p+\frac{v_{1}+v_{2}+v_{3}}{2}, \\
& p \simeq p+\frac{v_{4}+v_{5}+v_{6}}{2},
\end{aligned}
$$

for any point $p$ on the torus, as depicted in figure 9 .

In the new coordinates, only six wrapping numbers occur,

$$
\pi^{\text {torus }}:=\left(\tilde{n}^{1} v_{1}+\tilde{m}^{1} v_{2}\right) \wedge\left(\tilde{n}^{2} v_{3}+\tilde{m}^{2} v_{4}\right) \wedge\left(\tilde{n}^{3} \pi_{5}+\tilde{m}^{3} \pi_{6}\right),
$$

in terms of which the bulk wrapping numbers defined in (2.14) and (2.16) can be rewritten as follows:

$$
\begin{array}{lrl}
A_{1} q^{1} & =-A_{3} q^{1}=-\left(\tilde{n}^{1}+\tilde{m}^{1}\right) \tilde{n}^{2} \tilde{n}^{3}, & B_{1} m^{1}=-B_{3} m^{1}=\tilde{n}^{1} \tilde{m}^{2}\left(\tilde{n}^{3}+\tilde{m}^{3}\right), \\
A_{1} r^{1}=-A_{3} r^{1}=-\left(\tilde{n}^{1}+\tilde{m}^{1}\right) \tilde{n}^{2}\left(\tilde{n}^{3}+\tilde{m}^{3}\right), & B_{1} n^{1}=-B_{3} n^{1}=\left(\tilde{n}^{1}+\tilde{m}^{1}\right) \tilde{m}^{2}\left(\tilde{n}^{3}+\tilde{m}^{3}\right), \\
A_{1} s^{1}=-A_{3} s^{1}=-\left(\tilde{n}^{1}+\tilde{m}^{1}\right) \tilde{n}^{2} \tilde{m}^{3}, & B_{1} p^{1}=-B_{3} p^{1}=\tilde{m}^{1} \tilde{m}^{2}\left(\tilde{n}^{3}+\tilde{m}^{3}\right), \\
A_{2} q^{1}=\left(\tilde{n}^{1}-\tilde{m}^{1}\right) \tilde{n}^{2} \tilde{n}^{3}, & B_{2} m^{1}=\tilde{n}^{1} \tilde{m}^{2}\left(-\tilde{n}^{3}+\tilde{m}^{3}\right), \\
A_{2} r^{1}=\left(\tilde{n}^{1}-\tilde{m}^{1}\right) \tilde{n}^{2}\left(\tilde{n}^{3}+\tilde{m}^{3}\right), & B_{2} n^{1}=\left(\tilde{n}^{1}+\tilde{m}^{1}\right) \tilde{m}^{2}\left(-\tilde{n}^{3}+\tilde{m}^{3}\right), \\
A_{2} s^{1}=\left(\tilde{n}^{1}-\tilde{m}^{1}\right) \tilde{n}^{2} \tilde{m}^{3}, & B_{2} p^{1}=\tilde{m}^{1} \tilde{m}^{2}\left(-\tilde{n}^{3}+\tilde{m}^{3}\right) .
\end{array}
$$

Note that due to the shift symmetry (4.5), the wrapping numbers $\tilde{n}^{2}$ and $\tilde{m}^{2}$ can also have half-integer values.

On the factorised lattice, the geometric difference between the length of the O6-planes on the AAB- and BAA-orientations becomes clear: for the $\mathbf{A A B}$-lattice, the $\mathcal{R}$-invariant O6-plane is placed along the axes $v_{1}-v_{2}$ and $v_{3}$ on $T_{(1)}^{2} \times T_{(2)}^{2}$ and therefore it passes through the points which are identified by the shift symmetry (4.5), but this is no longer the case for the BAA-lattice.

$\boldsymbol{A}_{\mathbf{3}} \times \boldsymbol{A}_{\mathbf{1}} \times \boldsymbol{B}_{\mathbf{2}}$. Here it suffices to introduce new directions only on $A_{3} \times A_{1}$ :

$$
\begin{array}{ll}
v_{1}:=\pi_{1}+\pi_{2}, & v_{3}:=\pi_{1}+\pi_{3}, \\
v_{2}:=\pi_{2}+\pi_{3}, & v_{4}:=\pi_{4} .
\end{array}
$$



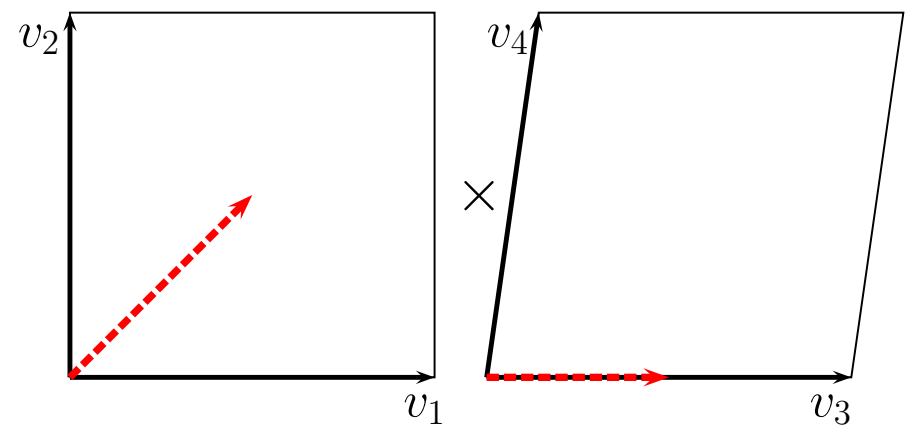

Figure 10. Shift symmetry of the factorised form of the $A_{3} \times A_{1}$-lattice along $\frac{v_{1}+v_{2}+v_{3}}{2}$ (in red).

This basis factorises the torus, such that the metric becomes

$$
g=\operatorname{diag}\left(\begin{array}{cc}
2(1+a) R_{3}^{2} & 0 \\
0 & 2(1+a) R_{3}^{2}
\end{array}\right) \oplus\left(\begin{array}{cc}
-4 a R_{3}^{2} & -4 a u_{1} R_{3}^{2} \\
-4 a u_{1} R_{3}^{2} & R_{1}^{2}
\end{array}\right) \oplus\left(\begin{array}{cc}
2 R_{2}^{2} & -R_{2}^{2} \\
-R_{2}^{2} & R_{2}^{2}
\end{array}\right) .
$$

For the Coxeter element $Q$ and the orientifold projections $\mathcal{R}_{i}$ we obtain

$$
\begin{gathered}
Q=\operatorname{diag}\left(\begin{array}{cc}
0 & -1 \\
1 & 0
\end{array}\right) \oplus\left(\begin{array}{cc}
-1 & 0 \\
0 & -1
\end{array}\right) \oplus\left(\begin{array}{ll}
1 & -1 \\
2 & -1
\end{array}\right), \\
\mathcal{R}_{1}=\operatorname{diag}\left(\begin{array}{cc}
0 & -1 \\
-1 & 0
\end{array}\right) \oplus\left(\begin{array}{ll}
1 & 2 u_{1} \\
0 & -1
\end{array}\right) \oplus\left(\begin{array}{ll}
1 & -1 \\
0 & -1
\end{array}\right), \\
\mathcal{R}_{4}=\operatorname{diag}\left(\begin{array}{cc}
-1 & 0 \\
0 & 1
\end{array}\right) \oplus\left(\begin{array}{ll}
1 & 2 u_{1} \\
0 & -1
\end{array}\right) \oplus\left(\begin{array}{ll}
1 & -1 \\
0 & -1
\end{array}\right) .
\end{gathered}
$$

Also in this case the basis change gives rise to an additional shift symmetry displayed in figure 10,

$$
p \simeq p+\frac{v_{1}+v_{2}+v_{3}}{2} \text { for any point } p \text { on the torus } .
$$

Instead of ten wrapping numbers which we need to describe a fractional three-cycle in non-factorisable coordinates, in the $v$-basis the usual six wrapping numbers are sufficient:

$$
\pi^{\text {torus }}:=\left(\tilde{n}^{1} v_{1}+\tilde{m}^{1} v_{2}\right) \wedge\left(\tilde{n}^{2} v_{3}+\tilde{m}^{2} v_{4}\right) \wedge\left(m^{3} \pi_{5}+n^{3} \pi_{6}\right)
$$

The relation between the non-factorised toroidal and bulk wrapping numbers in (2.26) and (2.29) and the new factorised ones is given by

$$
\begin{array}{ll}
A_{1}=-A_{3}=-\left(\tilde{n}^{1}+\tilde{m}^{1}\right) \tilde{n}^{2}, & A_{2}=\left(\tilde{n}^{1}-\tilde{m}^{1}\right) \tilde{n}^{2}, \\
B_{1}=\tilde{n}^{1} \tilde{m}^{2}, & B_{3}=\tilde{m}^{1} \tilde{m}^{2}=B_{2}-B_{1} .
\end{array}
$$

Notice that here, due to the shift symmetry (4.13), the wrapping number $\tilde{n}^{2}$ can be halfinteger if $\tilde{m}^{1}+\tilde{n}^{1}$ is even. 


\begin{tabular}{|c|c|c|}
\hline \multicolumn{3}{|c|}{$A_{3} \times A_{1} \times B_{2}$} \\
\hline & Two generations & Four generations \\
\hline $\mathbf{A}_{\mathbf{a}} \mathbf{A B}$ & $\infty, 1$ & $\infty, 1$ \\
\hline $\mathbf{A}_{\mathbf{b}} \mathbf{A B}$ & $\infty$ & $\infty$ \\
\hline $\mathbf{A}_{\mathbf{a}} \mathbf{B B}$ & $\infty, 1,3, \frac{1}{2}, \frac{1}{3}, \frac{1}{4}, \frac{1}{6}, \frac{1}{8}, \frac{1}{9}, \frac{1}{12}$ & $\infty, 1,2, \frac{1}{2}, \frac{1}{3}, \frac{1}{4}, \frac{1}{6}, \frac{1}{9}, \frac{1}{12}$ \\
\hline $\mathbf{A}_{\mathbf{b}} \mathbf{B B}$ & $\infty, 1, \frac{1}{4}$ & - \\
\hline
\end{tabular}

Table 17. The possible values of $\operatorname{Im}(\mathcal{U})$ for global supersymmetric Pati-Salam models, with $\infty$ denoting that it can be choosen arbitrarily.

\section{Concrete Pati-Salam models on $A_{3} \times A_{1} \times B_{2}$}

In this section we present the construction of some local and global semi-realistic supersymmetric Pati-Salam (PS) models using intersecting D6-branes. ${ }^{9}$ In section 3.3 we have seen that there exist duality relations between the different orientifold projections $\mathcal{R}_{i}$ $(i=1,2,3,4)$ and that only four lattice orientations are independent. For the construction of particle spectra, we choose the AAB- and ABB-types lattices. In addition to the $\mathrm{RR}$ tadpole cancellation and $s \mathrm{Lag}$ conditions, we require that the $\mathrm{U}(4)_{a}$ stack is free from (anti-)symmetric representations. ${ }^{10}$ An intensive computer search showed that only global supersymmetric PS-models with two or four generations are possible. The supersymmetry conditions give rise to restrictions on the allowed values of the imaginary part $u_{2}$ of the complex structure modulus $\mathcal{U}$, and only a small number of values can be used for the construction of global PS-models without overshooting the bulk RR tadpole cancellation condition. The allowed values of $u_{2}$ are illustrated in the table 17. For some branes, some bulk wrapping numbers turn out to be zero, and thus the supersymmetry conditions do not fix the complex structure. If PS-models can be constructed just with such branes, we will list the arbitrariness in $\operatorname{Im}(\mathcal{U})$ as $\infty$ in table 17.

Two generations. We start the search for global PS-models with the case of two particle generations and note some general properties of the models obtained. All models with $\mathrm{U}(4) \times \mathrm{U}(2) \times \mathrm{U}(2)$-gauge group in the visible sector contain chiral particles which transform in the (anti-)symmetric representation of the $\mathrm{U}(2)$ on the $b$ and/or $c$-stack. The spectrum of the models with gauge symmetry enhancement $\mathrm{U}(1) \hookrightarrow \mathrm{USp} / \mathrm{SO}(2)$ on both the $b$ - and $c$-stack, on the other hand, lacks the bifundamental representations in the $b c$-sector, i.e., there is no Higgs field in the chiral spectrum of such models. In table 18, we provide an explicit example of a PS-model with $\mathrm{U}(4) \times \mathrm{U}(2) \times \mathrm{USp} / \mathrm{SO}(2)$ gauge group in the visible sector. Its chiral spectrum is given in the table 19 .

\footnotetext{
${ }^{9}$ Notice that for generic configurations of gauge groups, global models do not only have to satisfy the RR tadpole cancellation conditions, but also the K-theory constraints, which are usually derived by scanning through all possible probe D-branes supporting USp(2) gauge factors [98]. For PS models with all gauge groups (including 'hidden' ones) of even rank, the K-theory constraints are, however, trivially fulfilled.

${ }^{10}$ The systematic computer search on the $A_{3} \times A_{1} \times B_{2}$ lattices actually showed that all two- and fourgeneration models of PS-type satisfy this condition without a priori imposing it.
} 


\begin{tabular}{|c|c|c|}
\hline \multicolumn{4}{|c|}{ D6-branes configuration for two generation PS-model on $A_{3} \times A_{1} \times B_{2}$-orientifold } \\
\hline Stack & $\left(m^{1}, n^{1}, p^{1}, q^{1}\right) \times\left(m^{2}, n^{2}, p^{2}, q^{2}\right) \times\left(m^{3}, n^{3}\right)$ & Homology cycle \\
\hline $\mathrm{U}(4)_{a}$ & $(1,0,-1,0) \times(1,0,0,1) \times(-1,-2)$ & $\frac{1}{2} \gamma_{1}-\frac{1}{2} \gamma_{2}-\bar{\gamma}_{1}+\bar{\gamma}_{2}$ \\
\hline $\mathrm{U}(2)_{b}$ & $(0,1,1,0) \times(0,0,0,1) \times(1,2)$ & $\bar{\gamma}_{2}-\bar{\gamma}_{3}+\bar{\gamma}_{4}$ \\
\hline $\mathrm{USp} / \mathrm{SO}(2)_{c}$ & $(1,1,0,0) \times(1,0,1,0) \times(-1,-2)$ & $\gamma_{1}+\gamma_{3}-\gamma_{4}$ \\
\hline \hline $\mathrm{USp} / \mathrm{SO}(2)_{h_{1}}$ & $(1,1,0,0) \times(1,0,1,0) \times(-1,-2)$ & $\gamma_{1}-\gamma_{3}+\gamma_{4}$ \\
\hline $\mathrm{U}(2)_{h_{2}}$ & $(0,1,1,0) \times(0,0,0,1) \times(1,2)$ & $\bar{\gamma}_{2}-\bar{\gamma}_{3}-\bar{\gamma}_{4}$ \\
\hline
\end{tabular}

Table 18. D6-branes for a two generation supersymmetric PS-model with $\mathbf{A}_{\mathbf{a}} \mathbf{B B}$-lattice and $u_{2}=1 / 2$.

\begin{tabular}{|c|c|}
\hline \multicolumn{2}{|c|}{ Chiral spectrum of two generation PS-model on $A_{3} \times A_{1} \times B_{2}$-orientifold } \\
\hline Sector & $\mathrm{SU}(4)_{a} \times \mathrm{SU}(2)_{b} \times \mathrm{USp} / \mathrm{SO}(2)_{c} \times \mathrm{USp} / \mathrm{SO}(2)_{h_{1}} \times \mathrm{SU}(2)_{h_{2}} \times \mathrm{U}(1)^{3}$ \\
\hline$a b$ & $(4, \overline{2}, 1,1,1)_{(1,-1,0)}$ \\
\hline$a b^{\prime}$ & $(4,2,1,1,1)_{(1,1,0)}$ \\
\hline$a c=a c^{\prime}$ & $2 \times(\overline{4}, 1,2,1,1)_{(-1,0,0)}$ \\
\hline$b c=b c^{\prime}$ & $4 \times(1,2, \overline{2}, 1,1)_{(0,1,0)}$ \\
\hline$a h_{1}=a h_{1}^{\prime}$ & $2 \times(\overline{4}, 1,1,2,1)_{(-1,0,0)}$ \\
\hline$b h_{1}=b h_{1}^{\prime}$ & $4 \times(1,2,1, \overline{2}, 1)_{(0,-1,0)}$ \\
\hline$a h_{2}$ & $(4,1,1,1, \overline{2})_{(1,0,-1)}$ \\
\hline$a h_{2}^{\prime}$ & $(4,1,1,1,2)_{(1,0,1)}$ \\
\hline
\end{tabular}

Table 19. Chiral spectrum for the two generation $\mathrm{U}(4) \times \mathrm{U}(2) \times \mathrm{USp} / \mathrm{SO}(2) \times \mathrm{USp} / \mathrm{SO}(2) \times \mathrm{U}(2)-\mathrm{PS}$ model with D6-brane configuration given in table 18.

In appendix $\mathrm{A}$, we provide an explicit example for each other type of visible gauge group, i.e. $\mathrm{U}(4) \times \mathrm{U}(2) \times \mathrm{U}(2)$ and $\mathrm{U}(4) \times \mathrm{USp} / \mathrm{SO}(2) \times \mathrm{USp} / \mathrm{SO}(2)$.

Four generations. In a similar way, we can realise global supersymmetric PS-models with four generations with different gauge groups (without/with some enhancement of the 'left' and 'right' stack) in the visible sector. In general, only the $\mathrm{U}(4) \times \mathrm{USp} / \mathrm{SO}(2) \times$ $\mathrm{USp} / \mathrm{SO}(2)$-models do not contain chiral matter in the (anti-)symmetric representation on the $b$ - and $c$ - stacks. But these models also do not contain chiral states in the bifundamental representation of the $b c$-sector, which could serve as a (chiral) Higgs multiplet. In appendix A, we collect several examples of global D6-brane configurations of such types together with their chiral spectrum.

Three generations. As mentioned above, there are no global supersymmetric PatiSalam models with three generations. But it is possible to construct models where the bulk part of the tadpole vanishes and only the exceptional part remains. Such local models are only realisable on the $\mathbf{A B B}$-orientifolds. More precisely, for the $\mathbf{A}_{\mathbf{a}} \mathbf{B B}$-lattice only $u_{2}=3, \frac{1}{3}$ provides such models, and the gauge group is enhanced on the $b$ - and $c$-stacks. 
For the $\mathbf{A}_{\mathbf{b}} \mathbf{B B}$-lattice, it is possible to construct local models with $u_{2}=\frac{3}{2}$ with symmetry enhancement on the $b$ - and/or $c$-stack. A general property of all such models is the appearance of chiral particles which transform in the (anti-)symmetric representation of $\mathrm{U}(4)_{a}$.

\section{Discussion and conclusions}

In this article, we explored the full three-cycle geometry of the non-factorisable $T^{6} / \mathbb{Z}_{4}$ orbifold on the two possible root lattices $A_{3} \times A_{3}$ and $A_{3} \times A_{1} \times B_{2}$ and compared it with the factorisable $B_{2} \times\left(A_{1}\right)^{2} \times B_{2}$ case. We found that, under the anti-holomorphic involution $\mathcal{R}$ of Type IIA $/ \Omega \mathcal{R}$ orientifolds, there exist a priori four different lattice orientations for $A_{3} \times A_{3}$, and eight for $A_{3} \times A_{1} \times B_{2}$. However, a closer look at the number of supersymmetric (i.e. $s L a g$ ) fractional three-cycles bounded in their length by the RR tadpole cancellation conditions and by the allowed values for the complex structure moduli, which encode the relative angles between the different root lattices as well as between the generators within each $A_{3}$ lattice, reveals - in analogy to the factorisable cases [42, 46, 48] — the existence of several dualities, which lead to identical physics for different choices of lattice orientations under $\mathcal{R}$. More precisely, on $A_{3} \times A_{3}$ we found a duality relation between the $\mathbf{A A A}$ and $\mathbf{B A B}$ lattices, leaving three independent choices. For the $A_{3} \times A_{1} \times B_{2}$ lattice, there are four pairwise duality relations $\left(\mathbf{A}_{\mathbf{a}} \mathbf{A} \mathbf{A}\right.$ and $\mathbf{\mathbf { A } _ { \mathbf { a } }} \mathbf{A B}, \mathbf{A}_{\mathbf{a}} \mathbf{B} \mathbf{A}$ and $\mathbf{A}_{\mathbf{a}} \mathbf{B B}, \mathbf{A}_{\mathbf{b}} \mathbf{A} \mathbf{A}$ and $\mathbf{A}_{\mathbf{b}} \mathbf{B B}, \mathbf{A}_{\mathbf{b}} \mathbf{B A}$ and $\mathbf{A}_{\mathbf{b}} \mathbf{A B}$ ), leaving only four independent choices.

After computing the sLag three-cycles, in order to ascertain our results, we crosschecked agreement of the RR tadpole cancellation conditions among our new purely geometric derivation as well as via the 'old' CFT method, in particular for the special D6-brane configurations on top of the O6-planes in [63], compared to which we generalised the CFT results to arbitrary values of the angles inside the $A_{3}$ lattices and the angle between the $A_{3}$ and the $A_{1}$ lattices. For the $A_{3} \times A_{3}$ lattices and the a-lattices of $A_{3} \times A_{1} \times B_{2}$, our results derived in a twofold, mutually agreeing way (mostly) coincide with the results listed in [63], while those corresponding to the b-type lattices of $A_{3} \times A_{1} \times B_{2}$ are newly found here.

With the full list of allowed $s L a g$ three-cycles at hand, we proceeded to search for local and global semi-realistic Pati-Salam models on both types of lattices. The $A_{3} \times A_{3}$ lattices happened to be very restricted - e.g. by the small number of available threecycles - and therefore unsuitable for model building. But the $A_{3} \times A_{1} \times B_{2}$ lattice with different orientations provided a very rich class of backgrounds with ample potential for model building. Although the search for global Pati-Salam models - to which we restricted ourselves here since the K-theory constraints are trivially fulfilled in that case - with three generations did not bear any fruit, many models with two and four generations were found. From a qualitative point of view, our results are in agreement with [99] — which studied these non-factorisable orbifolds in the context of the heterotic string - in the sense that the $A_{3} \times A_{1} \times B_{2}$ lattice is the most promising non-factorisable one for model building.

Our first model searches typically lead to globally consistent models, where one or more stacks of D-branes wrap three-cycles invariant under the anti-holomorphic involution $\mathcal{R}$. While it is well known that in such cases an enhancement of the gauge group 
$\mathrm{U}(N) \hookrightarrow \mathrm{USp}(2 N)$ or $\mathrm{SO}(2 N)$ occurs, the correct distinction can - with the generally available model building techniques to date - only be done by computing open string CFT amplitudes such as the gauge thresholds for factorisable backgrounds in $[45,46,48,83]$ and reading off the correct sign for the orientifold projection from the Möbius strip contribution to either the one-loop beta function coefficient or to the RR tadpoles. Our finding in section 4, that fractional three-cycles can be rewritten in a factorised form, suggests that the CFT methods can be straightforwardly generalised to the non-factorisable backgrounds of $T^{6} / \mathbb{Z}_{4}$ discussed here from a purely geometric viewpoint. By fully developing the corresponding CFT, not only the compete chiral plus vector-like matter spectrum can be determined, but also the low-energy effective action can (in principle) be derived. Identifying all probe D-branes supporting $\operatorname{USp}(2)$ gauge groups by means of CFT techniques is furthermore necessary to determine the K-theory constraints for all future Standard Model or GUT model building.

Last but not least, it will be interesting to extend the methods for studying nonfactorisable orbifolds to other point groups $\mathbb{Z}_{N \neq 4}$, and to incorporate closed string fluxes and study if particle physics models with (nearly) complete stabilisation of the closed string moduli are within reach.

\section{Acknowledgments}

This work is partially supported by the Cluster of Excellence 'Precision Physics, Fundamental Interactions and Structure of Matter' (PRISMA) DGF no. EXC 1098, the DFG research grant HO 4166/2-2, and the DFG Research Training Group 'Symmetry Breaking in Fundamental Interactions' GRK 1581.

\section{A More Pati-Salam models}

In this appendix, we provide additional explicit examples of global Pati-Salam models with two and four generations on the $A_{3} \times A_{1} \times B_{2}$ lattice.

\section{A.1 2 generations}

In the main text, we presented a global model where one of the left- or right-symmetric groups of the Pati-Salam gauge group is provided by an enhanced $\mathrm{SO}(2)_{L / R}$ or $\operatorname{USp}(2)_{L / R}$ symmetry. We also found global models where none or both left- and/or right-symmetric groups are replaced by an enhanced gauge symmetry; we will show an example of each of type in the following.

Tables 20 and 21 show the D6-branes and chiral spectrum, respectively, of a global Pati-Salam model with visible gauge group $\mathrm{U}(4) \times \mathrm{U}(2) \times \mathrm{U}(2)$.

Tables 22 and 23 show the D6-branes and chiral spectrum, respectively, of a global Pati-Salam model with visible gauge group $\mathrm{U}(4) \times \mathrm{USp} / \mathrm{SO}(2) \times \mathrm{USp} / \mathrm{SO}(2)$. 


\begin{tabular}{|c|c|c|}
\hline \multicolumn{2}{|c|}{ D6-branes configuration for a two generation PS-model on $A_{3} \times A_{1} \times B_{2}$-orientifold } \\
\hline $\mathrm{Stack}$ & $\left(m^{1}, n^{1}, p^{1}, q^{1}\right) \times\left(m^{2}, n^{2}, p^{2}, q^{2}\right) \times\left(m^{3}, n^{3}\right)$ & Homology cycle \\
\hline $\mathrm{U}(4)_{a}$ & $(1,2,1,0) \times(0,0,0,1) \times(0,1)$ & $\bar{\gamma}_{2}+\bar{\gamma}_{3}$ \\
\hline $\mathrm{U}(2)_{b}$ & $(1,1,0,0) \times(1,0,1,-1) \times(0,-1)$ & $\frac{1}{2}\left(\gamma_{1}+\gamma_{2}-\gamma_{3}-\gamma_{4}+\bar{\gamma}_{1}+\bar{\gamma}_{2}+\bar{\gamma}_{3}+\bar{\gamma}_{4}\right)$ \\
\hline $\mathrm{U}(2)_{c}$ & $(1,1,0,0) \times(1,0,1,-1) \times(0,-1)$ & $\frac{1}{2}\left(\gamma_{1}+\gamma_{2}+\gamma_{3}+\gamma_{4}+\bar{\gamma}_{1}+\bar{\gamma}_{2}-\bar{\gamma}_{3}-\bar{\gamma}_{4}\right)$ \\
\hline \hline $\mathrm{U}(2)_{h_{1}}$ & $(0,1,1,0) \times(0,0,0,1) \times(1,2)$ & $\bar{\gamma}_{2}-\bar{\gamma}_{3}+\bar{\gamma}_{4}$ \\
\hline $\mathrm{USp} / \mathrm{SO}(4)_{h_{2}}$ & $(1,1,0,0) \times(1,0,1,0) \times(-1,-2)$ & $\gamma_{1}$ \\
\hline
\end{tabular}

Table 20. D6-branes for a global two generation PS-model on the $\mathbf{A}_{\mathbf{a}} \mathbf{B B}$-lattice with $u_{2}=1$.

\begin{tabular}{|c|c|}
\hline \multicolumn{2}{|c|}{ Chiral spectrum of a two generation PS-model on $A_{3} \times A_{1} \times B_{2}$-orientifold } \\
\hline Sector & $\mathrm{SU}(4)_{a} \times \mathrm{SU}(2)_{b} \times \mathrm{SU}(2)_{c} \times \mathrm{SU}(2)_{h_{1}} \times \mathrm{USp} / \mathrm{SO}(4)_{h_{2}} \times \mathrm{U}(1)^{4}$ \\
\hline$a b$ & $2 \times(4, \overline{2}, 1,1,1)_{(1,-1,0,0)}$ \\
\hline$a c^{\prime}$ & $2 \times(\overline{4}, 1, \overline{2}, 1,1)_{(-1,0,-1,0)}$ \\
\hline$b c^{\prime}$ & $2 \times(1, \overline{2}, \overline{2}, 1,1)_{(0,-1,-1,0)}$ \\
\hline$b b^{\prime}$ & $2 \times(1,3,1,1,1)_{(0,0,0,0)}$ \\
\hline$c c^{\prime}$ & $2 \times(1,1,3,1,1)_{(0,0,0,0)}$ \\
\hline$b h_{1}^{\prime}$ & $2 \times(1, \overline{2}, 1, \overline{2}, 1)_{(0,-1,0,-1)}$ \\
\hline$c h_{1}$ & $2 \times(1,1, \overline{2}, 2,1)_{(0,0,-1,1)}$ \\
\hline$b h_{2}=b h_{2}^{\prime}$ & $(1,2,1,1, \overline{4})_{(0,1,0,0)}$ \\
\hline$c h_{2}=c h_{2}^{\prime}$ & $(1,1,2,1, \overline{4})_{(0,0,1,0)}$ \\
\hline
\end{tabular}

Table 21. Chiral spectrum of the two generation $\mathrm{U}(4) \times \mathrm{U}(2) \times \mathrm{U}(2) \times \mathrm{U}(2) \times \mathrm{USp} / \mathrm{SO}(4)-\mathrm{PS}$ model with D-brane configuration displayed in table 20.

\begin{tabular}{|c|c|c|}
\hline \multicolumn{2}{|c|}{ D6-branes configuration for a two generation $P S$-model on $A_{3} \times A_{1} \times B_{2}$-orientifold } \\
\hline Stack & $\left(m^{1}, n^{1}, p^{1}, q^{1}\right) \times\left(m^{2}, n^{2}, p^{2}, q^{2}\right) \times\left(m^{3}, n^{3}\right)$ & Homology cycle \\
\hline $\mathrm{U}(4)_{a}$ & $(1,2,1,0) \times(0,0,0,1) \times(0,1)$ & $\bar{\gamma}_{2}-\bar{\gamma}_{4}$ \\
\hline $\mathrm{USp} / \mathrm{SO}(2)_{b}$ & $(1,1,0,0) \times(1,0,1,0) \times(-1,-2)$ & $\gamma_{1}+\gamma_{3}+\gamma_{4}$ \\
\hline $\mathrm{USp} / \mathrm{SO}(2)_{c}$ & $(1,1,0,0) \times(1,0,1,0) \times(-1,-2)$ & $\gamma_{1}-\gamma_{3}-\gamma_{4}$ \\
\hline \hline $\mathrm{U}(4)_{h_{1}}$ & $(1,2,1,0) \times(0,0,0,1) \times(0,1)$ & $\bar{\gamma}_{2}+\bar{\gamma}_{4}$ \\
\hline $\mathrm{USp} / \mathrm{SO}(2)_{h_{2}}$ & $(1,1,0,0) \times(1,0,1,0) \times(-1,-2)$ & $\gamma_{1}$ \\
\hline
\end{tabular}

Table 22. D6-branes for a global two generation PS-model on the $\mathbf{A}_{\mathbf{a}} \mathbf{B B}$-lattice with non-fixed $u_{2}$.

\section{A.2 4 generations}

As in the previous case, we also found four-generation global Pati-Salam models where none, one or both left- and/or right-symmetric groups are replaced by an enhanced symmetry $\mathrm{SO}(2)_{L / R}$ or $\operatorname{USp}(2)_{L / R}$. Here we will show a concrete example for each of these three cases. 


\begin{tabular}{|c|c|}
\hline \multicolumn{2}{|c|}{ Chiral spectrum of a two generation PS-model on $A_{3} \times A_{1} \times B_{2}$-orientifold } \\
\hline Sector & $\mathrm{SU}(4)_{a} \times \mathrm{USp} / \mathrm{SO}(2)_{b} \times \mathrm{USp} / \mathrm{SO}(2)_{c} \times \mathrm{SU}(4)_{h_{1}} \times \mathrm{USp} / \mathrm{SO}(2)_{h_{2}} \times \mathrm{U}(1)^{2}$ \\
\hline$a b=a b^{\prime}$ & $2 \times(4, \overline{2}, 1,1,1)_{(1,0)}$ \\
\hline$a c=a c^{\prime}$ & $2 \times(\overline{4}, 1,2,1,1)_{(-1,0)}$ \\
\hline
\end{tabular}

Table 23. Chiral spectrum of the two generation $\mathrm{U}(4) \times \mathrm{USp} / \mathrm{SO}(2) \times \mathrm{USp} / \mathrm{SO}(2) \times \mathrm{USp} / \mathrm{SO}(2) \times$ U(4)-PS model with D6-brane configuration displayed in table 22.

\begin{tabular}{|c|c|c|}
\hline \multicolumn{2}{|c|}{ D6-branes configuration for a four generation PS-model on $A_{3} \times A_{1} \times B_{2}$-orientifold } \\
\hline Stack & $\left(m^{1}, n^{1}, p^{1}, q^{1}\right) \times\left(m^{2}, n^{2}, p^{2}, q^{2}\right) \times\left(m^{3}, n^{3}\right)$ & Homology cycle \\
\hline $\mathrm{U}(4)$ & $(0,1,1,0) \times(0,0,0,1) \times(1,2)$ & $\bar{\gamma}_{2}-\bar{\gamma}_{3}-\bar{\gamma}_{4}$ \\
\hline $\mathrm{U}(2)$ & $(0,1,1,0) \times(1,0,1,-1) \times(0,1)$ & $\frac{1}{2}\left(\gamma_{1}-\gamma_{2}+\gamma_{3}+\gamma_{4}-\bar{\gamma}_{1}+\bar{\gamma}_{2}+\bar{\gamma}_{3}+\bar{\gamma}_{4}\right)$ \\
\hline $\mathrm{U}(2)$ & $(1,1,0,0) \times(1,0,1,-1) \times(0,-1)$ & $\frac{1}{2}\left(\gamma_{1}+\gamma_{2}-\gamma_{3}-\gamma_{4}+\bar{\gamma}_{1}+\bar{\gamma}_{2}+\bar{\gamma}_{3}+\bar{\gamma}_{4}\right)$ \\
\hline \hline $\mathrm{U}(2)_{h_{1}}$ & $(0,1,1,0) \times(0,0,0,1) \times(1,2)$ & $\bar{\gamma}_{2}-\bar{\gamma}_{3}+\bar{\gamma}_{4}$ \\
\hline $\mathrm{USp} / \mathrm{SO}(4)_{h_{2}}$ & $(1,1,0,0) \times(1,0,1,0) \times(-1,-2)$ & $\gamma_{1}$ \\
\hline
\end{tabular}

Table 24. D6-branes for a global four generation PS-model on the $\mathbf{A}_{\mathbf{a}} \mathbf{B B}$-lattice with $u_{2}=1$.

\begin{tabular}{|c|c|}
\hline \multicolumn{2}{|c|}{ Chiral spectrum of a four generation PS-model on $A_{3} \times A_{1} \times B_{2}$-orientifold } \\
\hline Sector & $\mathrm{SU}(4)_{a} \times \mathrm{SU}(2)_{b} \times \mathrm{SU}(2)_{c} \times \mathrm{SU}(2)_{h_{1}} \times \mathrm{USp} / \mathrm{SO}(4)_{h_{2}} \times \mathrm{U}(1)^{4}$ \\
\hline$a b$ & $(4, \overline{2}, 1,1,1)_{(1,-1,0,0)}$ \\
\hline$a b^{\prime}$ & $3 \times(4,2,1,1,1)_{(1,1,0,0)}$ \\
\hline$a c$ & $(\overline{4}, 1,2,1,1)_{(-1,0,1,0)}$ \\
\hline$a c^{\prime}$ & $3 \times(\overline{4}, 1, \overline{2}, 1,1)_{(-1,0,-1,0)}$ \\
\hline$b c$ & $2 \times(1,2, \overline{2}, 1,1)_{(0,1,-1,0)}$ \\
\hline$b b^{\prime}$ & $2 \times(1,3,1,1,1)_{(0,0,0,0)}$ \\
\hline$c c^{\prime}$ & $2 \times(1,1,3,1,1)_{(0,0,0,0)}$ \\
\hline$b h_{1}^{\prime}$ & $2 \times(1, \overline{2}, 1, \overline{2}, 1)_{(0,-1,0,-1)}$ \\
\hline$c h_{1}$ & $(1,1, \overline{2}, 2,1)_{(0,0,-1,1)}$ \\
\hline$b h_{2}=b h_{2}^{\prime}$ & $(1,1,2,1, \overline{4})_{(0,1,0,0)}$ \\
\hline$c h_{2}=c h_{2}^{\prime}$ & \\
\hline
\end{tabular}

Table 25. Chiral spectrum of the four generation $\mathrm{U}(4) \times \mathrm{U}(2) \times \mathrm{U}(2) \times \mathrm{USp} / \mathrm{SO}(4) \times \mathrm{U}(2)-\mathrm{PS}$ model with D6-brane configuration displayed in table 24.

Tables 24 and 25 show the D6-brane configuration and chiral spectrum, respectively, of a Pati-Salam model with visible sector $\mathrm{U}(4) \times \mathrm{U}(2) \times \mathrm{U}(2)$.

Tables 26 and 27 show the D6-brane configuration and chiral spectrum, respectively, of a Pati-Salam model with visible sector $\mathrm{U}(4) \times \mathrm{U}(2) \times \mathrm{USp} / \mathrm{SO}(2)$.

Tables 28 and 29 show the D6-brane configuration and chiral spectrum, respectively, of a Pati-Salam model with visible sector $\mathrm{U}(4) \times \mathrm{USp} / \mathrm{SO}(2) \times \mathrm{USp} / \mathrm{SO}(2)$. 


\begin{tabular}{|c|c|c|}
\hline \multicolumn{2}{|c|}{ D6-branes configuration a for four generation PS-model on $A_{3} \times A_{1} \times B_{2}$-orientifold } \\
\hline Stack & $\left(m^{1}, n^{1}, p^{1}, q^{1}\right) \times\left(m^{2}, n^{2}, p^{2}, q^{2}\right) \times\left(m^{3}, n^{3}\right)$ & Homology cycle \\
\hline $\mathrm{U}(4)_{a}$ & $(0,1,1,0) \times(0,0,0,1) \times(1,2)$ & $\bar{\gamma}_{2}+\bar{\gamma}_{3}+\bar{\gamma}_{4}$ \\
\hline $\mathrm{U}(2)_{b}$ & $(0,1,1,0) \times(1,0,1,1) \times(0,1)$ & $\frac{1}{2}\left(\gamma_{1}-\gamma_{2}-\gamma_{3}-\gamma_{4}-\bar{\gamma}_{1}+\bar{\gamma}_{2}-\bar{\gamma}_{3}-\bar{\gamma}_{4}\right)$ \\
\hline $\mathrm{USp} / \mathrm{SO}(2)_{c}$ & $(1,1,0,0) \times(1,0,1,0) \times(-1,-2)$ & $\gamma_{1}+\gamma_{3}+\gamma_{4}$ \\
\hline \hline $\mathrm{U}(3)_{h_{1}}$ & $(0,1,1,0) \times(0,0,0,1) \times(1,2)$ & $\bar{\gamma}_{2}+\bar{\gamma}_{3}-\bar{\gamma}_{4}$ \\
\hline $\mathrm{USp} / \mathrm{SO}(2)_{h_{2}}$ & $(1,1,0,0) \times(1,0,1,0) \times(-1,-2)$ & $\gamma_{1}$ \\
\hline
\end{tabular}

Table 26. D6-branes for a global four generation PS-model on the $\mathbf{A}_{\mathbf{a}} \mathbf{B B}$-lattice with $u_{2}=1$.

\begin{tabular}{|c|c|}
\hline \multicolumn{2}{|c|}{ Chiral spectrum of a four generation PS-model on $A_{3} \times A_{1} \times B_{2}$-orientifold } \\
\hline Sector & $\mathrm{SU}(4)_{a} \times \mathrm{SU}(2)_{b} \times \mathrm{USp} / \mathrm{SO}(2)_{c} \times \mathrm{SU}(3)_{h_{1}} \times \mathrm{USp} / \mathrm{SO}(2)_{h_{2}} \times \mathrm{U}(1)^{3}$ \\
\hline$a b$ & $(4, \overline{2}, 1,1,1)_{(1,-1,0)}$ \\
\hline$a b^{\prime}$ & $3 \times(4,2,1,1,1)_{(1,1,0)}$ \\
\hline$a c=a c^{\prime}$ & $4 \times(\overline{4}, 1,2,1,1)_{(-1,0,0)}$ \\
\hline$b c=b c^{\prime}$ & $(1,2, \overline{2}, 1,1)_{(0,1,0)}$ \\
\hline$b b^{\prime}$ & $2 \times(1,3,1,1,1)_{(0,0,0)}$ \\
\hline$b h_{1}$ & $(1,2,1, \overline{3}, 1)_{(0,1,-1)}$ \\
\hline$b h_{1}^{\prime}$ & $(1,2,1,3,1)_{(0,1,1)}$ \\
\hline
\end{tabular}

Table 27. Chiral spectrum of the four generation $\mathrm{U}(4) \times \mathrm{U}(2) \times \mathrm{USp} / \mathrm{SO}(2) \times \mathrm{U}(3) \times \mathrm{USp} / \mathrm{SO}(2)-\mathrm{PS}$ model with D6-brane configuration given in table 26.

\begin{tabular}{|c|c|c|}
\hline \multicolumn{3}{|c|}{ D6-branes configuration for a four generation PS-model on $A_{3} \times A_{1} \times B_{2}$-orientifold } \\
\hline Stack & $\left(m^{1}, n^{1}, p^{1}, q^{1}\right) \times\left(m^{2}, n^{2}, p^{2}, q^{2}\right) \times\left(m^{3}, n^{3}\right)$ & Homology cycle \\
\hline $\mathrm{U}(4)_{a}$ & $(1,0,-1,0) \times(0,0,0,1) \times(-1,-2)$ & $-\bar{\gamma}_{1}+\bar{\gamma}_{2}+2 \bar{\gamma}_{4}$ \\
\hline $\mathrm{USp} / \mathrm{SO}(2)_{b}$ & $(0,1,0,0) \times(1,0,1,0) \times(1,2)$ & $\frac{1}{2} \gamma_{1}+\frac{1}{2} \gamma_{2}-\gamma_{4}$ \\
\hline $\mathrm{USp} / \mathrm{SO}(2)_{c}$ & $(0,1,0,0) \times(1,0,1,0) \times(1,2)$ & $\frac{1}{2} \gamma_{1}+\frac{1}{2} \gamma_{2}+\gamma_{4}$ \\
\hline \hline $\mathrm{USp} / \mathrm{SO}(4)_{h}$ & $(0,1,0,0) \times(1,0,1,0) \times(1,2)$ & $\frac{1}{2} \gamma_{1}+\frac{1}{2} \gamma_{2}$ \\
\hline
\end{tabular}

Table 28. D6-branes for a global four generation PS-model one the $\mathbf{A}_{\mathbf{a}} \mathbf{A B}$-lattice with nonfixed $u_{2}$.

\begin{tabular}{|c|c|}
\hline \multicolumn{2}{|c|}{ Chiral spectrum of a four generation PS-model on $A_{3} \times A_{1} \times B_{2}$-orientifold } \\
\hline Sector & $\mathrm{SU}(4)_{a} \times \mathrm{USp} / \mathrm{SO}(2)_{b} \times \mathrm{USp} / \mathrm{SO}(2)_{c} \times \mathrm{USp} / \mathrm{SO}(4)_{h} \times \mathrm{U}(1)$ \\
\hline$a b=a b^{\prime}$ & $4 \times(4, \overline{2}, 1,1)_{+1}$ \\
\hline$a c=a c^{\prime}$ & $4 \times(\overline{4}, 1,2,1)_{-1}$ \\
\hline
\end{tabular}

Table 29. Chiral spectrum of the four generation $\mathrm{U}(4) \times \mathrm{USp} / \mathrm{SO}(2) \times \mathrm{USp} / \mathrm{SO}(2) \times \mathrm{USp} / \mathrm{SO}(4)-\mathrm{PS}$ model with D6-brane configuration displayed in table 28. 
Open Access. This article is distributed under the terms of the Creative Commons Attribution License (CC-BY 4.0), which permits any use, distribution and reproduction in any medium, provided the original author(s) and source are credited.

\section{References}

[1] P. Candelas, G.T. Horowitz, A. Strominger and E. Witten, Vacuum configurations for superstrings, Nucl. Phys. B 258 (1985) 46 [INSPIRE].

[2] E. Witten, Symmetry breaking patterns in superstring models, Nucl. Phys. B 258 (1985) 75 [INSPIRE].

[3] L.E. Ibáñez, J.E. Kim, H.P. Nilles and F. Quevedo, Orbifold compactifications with three families of $\mathrm{SU}(3) \times \mathrm{SU}(2) \times \mathrm{U}(1)^{n}$, Phys. Lett. B 191 (1987) 282 [InSPIRE].

[4] O. Lebedev et al., A mini-landscape of exact MSSM spectra in heterotic orbifolds, Phys. Lett. B 645 (2007) 88 [hep-th/0611095] [INSPIRE].

[5] O. Lebedev, H.P. Nilles, S. Ramos-Sanchez, M. Ratz and P.K.S. Vaudrevange, Heterotic mini-landscape (II): completing the search for MSSM vacua in a $Z_{6}$ orbifold, Phys. Lett. B 668 (2008) 331 [arXiv:0807.4384] [INSPIRE].

[6] H.P. Nilles, S. Ramos-Sanchez, P.K.S. Vaudrevange and A. Wingerter, The orbifolder: a tool to study the low energy effective theory of heterotic orbifolds, Comput. Phys. Commun. 183 (2012) 1363 [arXiv:1110.5229] [INSPIRE].

[7] K.-S. Choi, S. Groot Nibbelink and M. Trapletti, Heterotic SO(32) model building in four dimensions, JHEP 12 (2004) 063 [hep-th/0410232] [INSPIRE].

[8] A. Bak, V. Bouchard and R. Donagi, Exploring a new peak in the heterotic landscape, JHEP 06 (2010) 108 [arXiv:0811.1242] [INSPIRE].

[9] L.B. Anderson, J. Gray, A. Lukas and E. Palti, Two hundred heterotic standard models on smooth Calabi-Yau threefolds, Phys. Rev. D 84 (2011) 106005 [arXiv:1106.4804] [InSPIRE].

[10] L.B. Anderson, J. Gray, A. Lukas and E. Palti, Heterotic line bundle standard models, JHEP 06 (2012) 113 [arXiv:1202.1757] [INSPIRE].

[11] L.B. Anderson, A. Constantin, J. Gray, A. Lukas and E. Palti, A comprehensive scan for heterotic SU(5) GUT models, JHEP 01 (2014) 047 [arXiv: 1307.4787] [INSPIRE].

[12] S. Groot Nibbelink, O. Loukas, F. Ruehle and P.K.S. Vaudrevange, Infinite number of MSSMs from heterotic line bundles?, Phys. Rev. D 92 (2015) 046002 [arXiv:1506.00879] [INSPIRE].

[13] J. Polchinski, Dirichlet branes and Ramond-Ramond charges, Phys. Rev. Lett. 75 (1995) 4724 [hep-th/9510017] [INSPIRE].

[14] C. Vafa and E. Witten, A strong coupling test of S duality, Nucl. Phys. B 431 (1994) 3 [hep-th/9408074] [INSPIRE].

[15] J. Polchinski and E. Witten, Evidence for heterotic-type I string duality, Nucl. Phys. B 460 (1996) 525 [hep-th/9510169] [INSPIRE].

[16] J.H. Schwarz, The power of M-theory, Phys. Lett. B 367 (1996) 97 [hep-th/9510086] [INSPIRE].

[17] C. Vafa, Evidence for F-theory, Nucl. Phys. B 469 (1996) 403 [hep-th/9602022] [INSPIRE]. 
[18] L.E. Ibáñez and A.M. Uranga, String theory and particle physics: an introduction to string phenomenology, Cambridge University Press, Cambridge U.K. (2012) [INSPIRE].

[19] D. Joyce, Lectures on Calabi-Yau and special Lagrangian geometry, math.DG/0108088 [INSPIRE].

[20] D. Joyce, Lectures on special Lagrangian geometry, math.DG/0111111.

[21] E. Palti, Model building with intersecting D6-branes on smooth Calabi-Yau manifolds, JHEP 04 (2009) 099 [arXiv: 0902.3546] [INSPIRE].

[22] L.E. Ibáñez, F. Marchesano and R. Rabadán, Getting just the standard model at intersecting branes, JHEP 11 (2001) 002 [hep-th/0105155] [INSPIRE].

[23] D. Cremades, L.E. Ibáñez and F. Marchesano, SUSY quivers, intersecting branes and the modest hierarchy problem, JHEP 07 (2002) 009 [hep-th/0201205] [INSPIRE].

[24] D. Cremades, L.E. Ibáñez and F. Marchesano, Intersecting brane models of particle physics and the Higgs mechanism, JHEP 07 (2002) 022 [hep-th/0203160] [INSPIRE].

[25] M. Cvetič, G. Shiu and A.M. Uranga, Three family supersymmetric standard-like models from intersecting brane worlds, Phys. Rev. Lett. 87 (2001) 201801 [hep-th/0107143] [INSPIRE].

[26] M. Cvetič, G. Shiu and A.M. Uranga, Chiral four-dimensional $N=1$ supersymmetric type $2 A$ orientifolds from intersecting D6 branes, Nucl. Phys. B 615 (2001) 3 [hep-th/0107166] [INSPIRE].

[27] R. Blumenhagen, M. Cvetič, P. Langacker and G. Shiu, Toward realistic intersecting D-brane models, Ann. Rev. Nucl. Part. Sci. 55 (2005) 71 [hep-th/0502005] [inSPIRE].

[28] F. Gmeiner, R. Blumenhagen, G. Honecker, D. Lüst and T. Weigand, One in a billion: MSSM-like D-brane statistics, JHEP 01 (2006) 004 [hep-th/0510170] [INSPIRE].

[29] R. Blumenhagen, B. Körs, D. Lüst and S. Stieberger, Four-dimensional string compactifications with D-branes, orientifolds and fluxes, Phys. Rept. 445 (2007) 1 [hep-th/0610327] [INSPIRE].

[30] F. Gmeiner and M. Stein, Statistics of SU(5) D-brane models on a type-II orientifold, Phys. Rev. D 73 (2006) 126008 [hep-th/0603019] [INSPIRE].

[31] G. Honecker, Chiral supersymmetric models on an orientifold of $Z_{4} \times Z_{2}$ with intersecting D6-branes, Nucl. Phys. B 666 (2003) 175 [hep-th/0303015] [INSPIRE].

[32] G. Honecker, Supersymmetric intersecting D6-branes and chiral models on the $T^{6} /\left(\mathbb{Z}_{4} \times \mathbb{Z}_{2}\right)$ orbifold, in Proceedings of String phenomenology. 2nd International Conference, Durham U.K., 29 Jul-4 Aug 2003, pp. 191-198.

[33] G. Honecker, Chiral $N=14 D$ orientifolds with D-branes at angles, Mod. Phys. Lett. A 19 (2004) 1863 [hep-th/0407181] [InSPIRE].

[34] R. Blumenhagen, L. Görlich and T. Ott, Supersymmetric intersecting branes on the type IIA $T^{6} / Z_{4}$ orientifold, JHEP 01 (2003) 021 [hep-th/0211059] [INSPIRE].

[35] G. Honecker and T. Ott, Getting just the supersymmetric standard model at intersecting branes on the $\mathbb{Z}_{6}$ orientifold, Phys. Rev. D 70 (2004) 126010

[Erratum ibid. D 71 (2005) 069902] [hep-th/0404055] [INSPIRE].

[36] F. Gmeiner, D. Lüst and M. Stein, Statistics of intersecting D-brane models on $T^{6} / \mathbb{Z}_{6}$, JHEP 05 (2007) 018 [hep-th/0703011] [INSPIRE]. 
[37] F. Gmeiner and G. Honecker, Complete gauge threshold corrections for intersecting fractional D6-branes: the $\mathbb{Z}_{6}$ and $\mathbb{Z}_{6}^{\prime}$ standard models, Nucl. Phys. B 829 (2010) 225 [arXiv:0910.0843] [INSPIRE].

[38] D. Bailin and A. Love, Towards the supersymmetric standard model from intersecting D6-branes on the $\mathbb{Z}_{6}^{\prime}$ orientifold, Nucl. Phys. B 755 (2006) 79 [Erratum ibid. B 783 (2007) 176] [hep-th/0603172] [INSPIRE].

[39] D. Bailin and A. Love, Almost the supersymmetric standard model from intersecting D6-branes on the $\mathbb{Z}_{6}^{\prime}$ orientifold, Phys. Lett. B 651 (2007) 324 [Erratum ibid. B 658 (2008) 292] [arXiv:0705.0646] [INSPIRE].

[40] F. Gmeiner and G. Honecker, Mapping an island in the landscape, JHEP 09 (2007) 128 [arXiv:0708.2285] [INSPIRE].

[41] D. Bailin and A. Love, Constructing the supersymmetric standard model from intersecting D6-branes on the $\mathbb{Z}_{6}^{\prime}$ orientifold, Nucl. Phys. B 809 (2009) 64 [arXiv:0801.3385] [InSPIRE].

[42] F. Gmeiner and G. Honecker, Millions of standard models on $\mathbb{Z}_{6}^{\prime}$ ?, JHEP 07 (2008) 052 [arXiv:0806.3039] [INSPIRE].

[43] D. Bailin and A. Love, Intersecting D6-branes on the $\mathbb{Z}_{12}$-II orientifold, JHEP 01 (2014) 009 [arXiv: 1310.8215] [INSPIRE].

[44] R. Blumenhagen, M. Cvetič, F. Marchesano and G. Shiu, Chiral D-brane models with frozen open string moduli, JHEP 03 (2005) 050 [hep-th/0502095] [INSPIRE].

[45] S. Förste and G. Honecker, Rigid D6-branes on $T^{6} /\left(\mathbb{Z}_{2} \times \mathbb{Z}_{2 M} \times \Omega \mathcal{R}\right)$ with discrete torsion, JHEP 01 (2011) 091 [arXiv: 1010.6070] [INSPIRE].

[46] G. Honecker, M. Ripka and W. Staessens, The importance of being rigid: D6-brane model building on $T^{6} / \mathbb{Z}_{2} \times \mathbb{Z}_{6}^{\prime}$ with discrete torsion, Nucl. Phys. B 868 (2013) 156 [arXiv: 1209.3010] [INSPIRE].

[47] G. Honecker and W. Staessens, D6-brane model building and discrete symmetries on $T^{6} /\left(\mathbb{Z}_{2} \times \mathbb{Z}_{6} \times \Omega \mathcal{R}\right)$ with discrete torsion, PoS (Corfu2012) 107 [arXiv:1303.6845] [INSPIRE].

[48] J. Ecker, G. Honecker and W. Staessens, Rigour and rigidity: systematics on particle physics D6-brane models on $\mathbb{Z}_{2} \times \mathbb{Z}_{6}$, Fortschr. Phys. 62 (2014) 981 [arXiv:1409.1236] [inSPIRE].

[49] J. Ecker, G. Honecker and W. Staessens, D6-brane model building on $\mathbb{Z}_{2} \times \mathbb{Z}_{6}$ : MSSM-like and left-right symmetric models, Nucl. Phys. B 901 (2015) 139 [arXiv:1509.00048] [INSPIRE].

[50] G. Honecker, From stringy particle physics to moduli stabilisation and cosmology, Fortschr. Phys. 64 (2016) 380 [arXiv:1510.08443] [INSPIRE].

[51] M. Blaszczyk, G. Honecker and I. Koltermann, Circling the square: deforming fractional

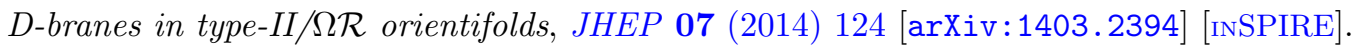

[52] M. Blaszczyk, G. Honecker and I. Koltermann, Deformations on tilted tori and moduli stabilisation at the orbifold point, JHEP 11 (2015) 019 [arXiv:1507.07568] [INSPIRE].

[53] I. Koltermann, M. Blaszczyk and G. Honecker, Deforming D-brane models on $T^{6} /\left(\mathbb{Z}_{2} \times \mathbb{Z}_{2 M}\right)$ orbifolds, Fortschr. Phys. 64 (2016) 412 [arXiv: 1511.03549] [INSPIRE].

[54] M. Graña, Flux compactifications in string theory: a comprehensive review, Phys. Rept. 423 (2006) 91 [hep-th/0509003] [INSPIRE]. 
[55] P. Koerber, Lectures on generalized complex geometry for physicists, Fortschr. Phys. 59 (2011) 169 [arXiv:1006.1536] [INSPIRE].

[56] M. Larfors, Generalised geometry and flux vacua, Fortschr. Phys. 64 (2016) 354 [arXiv: 1511.04595] [INSPIRE].

[57] M. Ihl and T. Wrase, Towards a realistic type IIA $T^{6} / \mathbb{Z}_{4}$ orientifold model with background fluxes. Part 1. Moduli stabilization, JHEP 07 (2006) 027 [hep-th/0604087] [INSPIRE].

[58] M. Ihl, D. Robbins and T. Wrase, Toroidal orientifolds in IIA with general NS-NS fluxes, JHEP 08 (2007) 043 [arXiv:0705.3410] [INSPIRE].

[59] D. Bailin and A. Love, Stabilising the supersymmetric standard model on the $\mathbb{Z}_{6}^{\prime}$ orientifold, Nucl. Phys. B 854 (2012) 700 [arXiv:1104.3522] [inSPIRE].

[60] P.G. Camara, A. Font and L.E. Ibáñez, Fluxes, moduli fixing and MSSM-like vacua in a simple IIA orientifold, JHEP 09 (2005) 013 [hep-th/0506066] [INSPIRE].

[61] G. Aldazabal, P.G. Camara, A. Font and L.E. Ibáñez, More dual fluxes and moduli fixing, JHEP 05 (2006) 070 [hep-th/0602089] [INSPIRE].

[62] F. Marchesano, D6-branes and torsion, JHEP 05 (2006) 019 [hep-th/0603210] [INSPIRE].

[63] R. Blumenhagen, J.P. Conlon and K. Suruliz, Type IIA orientifolds on general supersymmetric $\mathbb{Z}_{N}$ orbifolds, JHEP 07 (2004) 022 [hep-th/0404254] [INSPIRE].

[64] T. Kimura, M. Ohta and K.-J. Takahashi, Type IIA orientifolds and orbifolds on non-factorizable tori, Nucl. Phys. B 798 (2008) 89 [arXiv:0712.2281] [INSPIRE].

[65] S. Förste, C. Timirgaziu and I. Zavala, Orientifold's landscape: non-factorisable six-tori, JHEP 10 (2007) 025 [arXiv:0707.0747] [INSPIRE].

[66] S. Förste and I. Zavala, Oddness from rigidness, JHEP 07 (2008) 086 [arXiv:0806.2328] [INSPIRE].

[67] S. Förste and C. Liyanage, Yukawa couplings for intersecting D-branes on non-factorisable tori, JHEP 03 (2015) 110 [arXiv: 1412.3645] [INSPIRE].

[68] A. Seifert and G. Honecker, Model building on the non-factorisable type IIA $T^{6} /\left(\mathbb{Z}_{4} \times \Omega \mathcal{R}\right)$ orientifold, Fortschr. Phys. 64 (2016) 416 [arXiv:1511.03075] [INSPIRE].

[69] T.P.T. Dijkstra, L.R. Huiszoon and A.N. Schellekens, Chiral supersymmetric standard model spectra from orientifolds of Gepner models, Phys. Lett. B 609 (2005) 408 [hep-th/0403196] [INSPIRE].

[70] T.P.T. Dijkstra, L.R. Huiszoon and A.N. Schellekens, Supersymmetric standard model spectra from RCFT orientifolds, Nucl. Phys. B $\mathbf{7 1 0}$ (2005) 3 [hep-th/0411129] [INSPIRE].

[71] P. Anastasopoulos, T.P.T. Dijkstra, E. Kiritsis and A.N. Schellekens, Orientifolds, hypercharge embeddings and the standard model, Nucl. Phys. B 759 (2006) 83 [hep-th/0605226] [INSPIRE].

[72] P. Anastasopoulos, G.K. Leontaris, R. Richter and A.N. Schellekens, SU(5) D-brane realizations, Yukawa couplings and proton stability, JHEP 12 (2010) 011 [arXiv:1010.5188] [INSPIRE].

[73] D. Lüst and S. Stieberger, Gauge threshold corrections in intersecting brane world models, Fortschr. Phys. 55 (2007) 427 [hep-th/0302221] [INSPIRE]. 
[74] S.A. Abel and A.W. Owen, Interactions in intersecting brane models, Nucl. Phys. B 663 (2003) 197 [hep-th/0303124] [InSPIRE].

[75] M. Cvetič and I. Papadimitriou, Conformal field theory couplings for intersecting D-branes on orientifolds, Phys. Rev. D 68 (2003) 046001 [Erratum ibid. D 70 (2004) 029903] [hep-th/0303083] [INSPIRE].

[76] S.A. Abel and A.W. Owen, $N$ point amplitudes in intersecting brane models, Nucl. Phys. B 682 (2004) 183 [hep-th/0310257] [InSPIRE].

[77] D. Lüst, P. Mayr, R. Richter and S. Stieberger, Scattering of gauge, matter and moduli fields from intersecting branes, Nucl. Phys. B 696 (2004) 205 [hep-th/0404134] [INSPIRE].

[78] N. Akerblom, R. Blumenhagen, D. Lüst and M. Schmidt-Sommerfeld, Instantons and holomorphic couplings in intersecting D-brane models, JHEP 08 (2007) 044 [arXiv:0705.2366] [INSPIRE].

[79] R. Blumenhagen and M. Schmidt-Sommerfeld, Gauge thresholds and Kähler metrics for rigid intersecting D-brane models, JHEP 12 (2007) 072 [arXiv:0711.0866] [INSPIRE].

[80] G. Honecker, Kähler metrics and gauge kinetic functions for intersecting D6-branes on toroidal orbifolds - the complete perturbative story, Fortschr. Phys. 60 (2012) 243 [arXiv:1109.3192] [INSPIRE].

[81] G. Honecker, Towards exact field theory results for the standard model on fractional D6-branes, PoS (EPS-HEP2011) 129 [arXiv:1109.6533] [INSPIRE].

[82] M. Berg, M. Haack and J.U. Kang, One-loop Kähler metric of D-branes at angles, JHEP 11 (2012) 091 [arXiv:1112.5156] [INSPIRE].

[83] G. Honecker and W. Staessens, To tilt or not to tilt: discrete gauge symmetries in global intersecting D-brane models, JHEP 10 (2013) 146 [arXiv:1303.4415] [INSPIRE].

[84] D. Lüst, S. Reffert, E. Scheidegger and S. Stieberger, Resolved toroidal orbifolds and their orientifolds, Adv. Theor. Math. Phys. 12 (2008) 67 [hep-th/0609014] [INSPIRE].

[85] M. Spalinski, Duality transformations in twisted Narain compactifications, Nucl. Phys. B 377 (1992) 339 [inSPIRE].

[86] R. Blumenhagen, V. Braun, B. Körs and D. Lüst, Orientifolds of K3 and Calabi-Yau manifolds with intersecting D-branes, JHEP 07 (2002) 026 [hep-th/0206038] [INSPIRE].

[87] M. Cvetič and I. Papadimitriou, More supersymmetric standard-like models from intersecting D6-branes on type IIA orientifolds, Phys. Rev. D 67 (2003) 126006 [hep-th/0303197] [INSPIRE].

[88] M. Cvetič, T. Li and T. Liu, Supersymmetric Pati-Salam models from intersecting D6-branes: a road to the standard model, Nucl. Phys. B 698 (2004) 163 [hep-th/0403061] [INSPIRE].

[89] C.-M. Chen, T. Li and D.V. Nanopoulos, Type IIA Pati-Salam flux vacua, Nucl. Phys. B 740 (2006) 79 [hep-th/0601064] [InSPIRE].

[90] L.J. Dixon, J.A. Harvey, C. Vafa and E. Witten, Strings on orbifolds, Nucl. Phys. B 261 (1985) 678 [inSPIRE].

[91] L.J. Dixon, J.A. Harvey, C. Vafa and E. Witten, Strings on orbifolds (II), Nucl. Phys. B 274 (1986) 285 [inSPIRE].

[92] R. Blumenhagen, L. Görlich and B. Körs, Supersymmetric orientifolds in $6 D$ with D-branes at angles, Nucl. Phys. B 569 (2000) 209 [hep-th/9908130] [INSPIRE]. 
[93] R. Blumenhagen, L. Görlich and B. Körs, Supersymmetric $4 D$ orientifolds of type IIA with D6-branes at angles, JHEP 01 (2000) 040 [hep-th/9912204] [INSPIRE].

[94] S. Förste, G. Honecker and R. Schreyer, Supersymmetric $\mathbb{Z}_{N} \times \mathbb{Z}_{M}$ orientifolds in $4 D$ with D-branes at angles, Nucl. Phys. B 593 (2001) 127 [hep-th/0008250] [INSPIRE].

[95] T.W. Grimm and J. Louis, The effective action of type IIA Calabi-Yau orientifolds, Nucl. Phys. B 718 (2005) 153 [hep-th/0412277] [INSPIRE].

[96] N. Akerblom, R. Blumenhagen, D. Lüst and M. Schmidt-Sommerfeld, Thresholds for intersecting D-branes revisited, Phys. Lett. B 652 (2007) 53 [arXiv:0705.2150] [InSPIRE].

[97] M. Blaszczyk, S. Groot Nibbelink and F. Ruehle, Gauged linear $\sigma$-models for toroidal orbifold resolutions, JHEP 05 (2012) 053 [arXiv:1111.5852] [INSPIRE].

[98] A.M. Uranga, D-brane probes, $R R$ tadpole cancellation and $k$-theory charge, Nucl. Phys. B 598 (2001) 225 [hep-th/0011048] [INSPIRE].

[99] H.P. Nilles and P.K.S. Vaudrevange, Geography of fields in extra dimensions: string theory lessons for particle physics, Mod. Phys. Lett. A 30 (2015) 1530008 [arXiv:1403.1597] [INSPIRE]. 